\title{
General aspects of heterotic string compactifications on stacks and gerbes
}

\author{
Lara B. Anderson, Bei Jia, Ryan Manion, \\ Burt Ovrut and ErIC Sharpe
}

\begin{abstract}
In this paper we work out some basic results concerning heterotic string compactifications on stacks and, in particular, gerbes. A heterotic string compactification on a gerbe can be understood as, simultaneously, both a compactification on a space with a restriction on nonperturbative sectors, and also, a gauge theory in which a subgroup of the gauge group acts trivially on the massless matter. Gerbes admit more bundles than corresponding spaces, which suggests they are potentially a rich playground for heterotic string compactifications. After we give a general characterization of heterotic strings on stacks, we specialize to gerbes, and consider three different classes of 'building blocks' of gerbe compactifications. We argue that heterotic string compactifications on one class is equivalent to compactification of the same heterotic string on a disjoint union of spaces, compactification on another class is dual to compactifications of other heterotic strings on spaces, and compactification on the third class is not perturbatively consistent, so that we do not in fact recover a broad array of new heterotic compactifications, just combinations of existing ones. In appendices we explain how to compute massless spectra of heterotic string compactifications on stacks, derive some new necessary conditions for a heterotic string on a stack or orbifold to be well-defined, and also review some basic properties of bundles on gerbes.
\end{abstract}

\begin{tabular}{lll}
\hline 2 & Generalities & 537
\end{tabular}

\begin{tabular}{lll}
\hline 2.1 & Strings on stacks & 537
\end{tabular}

\begin{tabular}{|ll}
2.2 & Review of gerbes \\
\hline
\end{tabular} 
\begin{tabular}{|ll}
2.3 & Notions of twisting
\end{tabular}

3 Class I: Gauge bundle a pullback from the base 544

3.1 Review of $(2,2)$ decomposition conjecture 544

\begin{tabular}{|ll}
3.2 & Heterotic decomposition conjecture \\
\hline
\end{tabular}

\begin{tabular}{lll}
\hline 4 Class II: Dualities & 548
\end{tabular}

\begin{tabular}{lll}
\hline 4.1 & Basic proposal & 548
\end{tabular}

4.2 Toroidal orbifold example $\quad 550$

\begin{tabular}{|ll}
4.3 & Examples in Distler-Kachru models \\
\hline
\end{tabular}

5 Class III: Twisted bundles 556

$\begin{array}{lll}5.1 \text { Motivations } & 558\end{array}$

5.2 Constructions of consistent CFT's 561

$\begin{array}{lll}5.3 & \text { Cautionary example } & 563\end{array}$

\begin{tabular}{|lll}
5.4 & Second cautionary example & 567
\end{tabular}

\begin{tabular}{|ll}
5.5 & Third cautionary example \\
\hline
\end{tabular}

$\begin{array}{lll}5.6 & \text { Potential refinements of anomaly cancellation } & 572\end{array}$

$\begin{array}{lll}6 & \text { Combinations } & 573\end{array}$

\begin{tabular}{lll}
\hline & Conclusions & 573
\end{tabular}

8 Acknowledgements $\quad 574$

\begin{tabular}{|ll}
\hline Appendix A Massless spectra of heterotic strings on stacks & 575
\end{tabular}

\begin{tabular}{ll}
\hline A.1 Basic definitions & 575
\end{tabular}

\begin{tabular}{|ll}
\hline A.2 Vacuum energies & 578
\end{tabular} 
A.4 Consistency conditions derived from existence of Fock vacua

A.5 Spectrum result and Serre duality

\section{Introduction}

The compactification of heterotic superstrings on smooth Calabi-Yau threefolds has led to realistic $N=1$ supersymmetric particle physics in fourdimensions. For the $E_{8} \times E_{8}$ heterotic string, the generic structure of such vacua was presented in [1 4]. Building upon these results, many phenomenologically relevant low-energy theories with MSSM-like matter spectra have been constructed, see for example [5-12] for constructions and related work. However, the limitation of these vacua to equivariant vector bundles over smooth Calabi-Yau manifolds seems overly restrictive, and it is of considerable interest to try to construct heterotic vacua over more general backgrounds.

The purpose of this paper is to outline basic results and general issues in making sense of heterotic string compactifications on stacks, generalized spaces admitting metrics, spinors, and all the other items needed to make sense of a string compactification. This essentially completes a program started many years ago to understand the basics of string compactifications on stacks, see e.g. 13 23]. The original hope of this program was to find new SCFT's, new string compactifications, arising from these generalized spaces. 
Although that has not proven to be the case, much has been learned about the structure of string compactifications, as we shall review.

One of the most physically interesting kinds of stacks are known as gerbes. The worldsheet theory of a string compactification on a gerbe can be understood in two more or les: $\mathrm{1}^{1}$ equivalent ways:

- as a sigma model on a space, but with a (combinatoria $2^{2}$ restriction on allowed nonperturbative sectors, or

- as a gauge theory in which a (finite) subgroup of the gauge group acts trivially on the massless matter.

Viewed from the first perspective, it is clear that there is a potential problem with cluster decomposition in these theories. For $(2,2)$ SCFT's, this issue was addressed in [17], where it was argued that the SCFT is equivalent to that on a disjoint union of spaces with variable $B$ fields, a result listed there as the 'decomposition conjecture.' A sigma model on a disjoint union also violates cluster decomposition, but in an extremely mild fashion, easily understood. This duality has since proven crucial for understanding physics issues in many GLSM's, see e.g. [18, 24-28], and also has been used to make predictions for Gromov-Witten invariants of gerbes, predictions which have been checked in e.g. [29 34].

Viewed from the second perspective, there are analogous issues concerning whether and how physics can see a trivially-acting finite group. This was addressed in [14 16, and will be reviewed later in this paper. Mass-

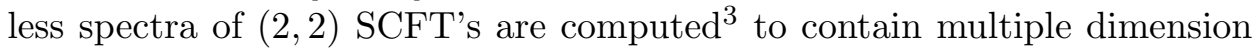
zero operators, another sign of cluster decomposition issues. These multiple dimension zero operators are (discrete Fourier transforms of) identity operators counting the number of components in the corresponding disjoint union of spaces [17].

1 Mathematically, the second description, as a gauge theory in which a finite subgroup acts trivially, implies the first, together with a small amount of additional information, a certain trivialization, which we have suppressed from the description of the first, so we should be slightly careful in claiming that they are precisely the same.

${ }^{2}$ Meaning, only instantons with degrees satisfying certain divisibility properties are included.

3 The papers [14 16] contain consistency checks of this computation. Ultimately, demanding modular invariance forces the spectrum to contain multiple dimension zero operators. 
These ideas have also been recently been applied to four-dimensional supergravity theories 36 39. For example, gerbes admit line bundles with fractional Chern classes, so the Bagger-Witten [40] quantization condition on cohomology classes of Kähler forms is modified when the supergravity moduli space admits a gerbe structure. More generally, a general introduction to four-dimensional supergravities whose moduli spaces are stacks (generic in Calabi-Yau compactification) is in [39]. Furthermore, it was shown in [41] [Appendix B] that four-dimensional supergravity anomalies have a natural description in terms of stacks. See for example [42, 43] for other applications.

This paper is concerned with heterotic string compactifications on stacks and, in particular, gerbes. As the introduction above alludes, there are many more bundles on gerbes than on corresponding spaces, which naively suggests that there could be a rich new landscape of $(0,2)$ SCFT's and heterotic string compactifications obtainable from heterotic compactifications on gerbes. Our results break into three fundamental building blocks or classes:

- For heterotic compactifications on gerbes in which the gauge bundle is a pullback from the base (equivalently, when the group that acts trivially on the base, also acts trivially on the bundle), the heterotic string compactification is consistent, and is equivalent to a compactification on a disjoint union of spaces. Compactifications of this form are discussed in Section 3 ,

- For heterotic compactifications on $\mathbb{Z}_{2}$ gerbes in which the $\mathbb{Z}_{2}$ acts nontrivially on a rank 8 bundle, these compactifications do not decompose, and (we conjecture) are T-dual to ordinary heterotic compactifications (on spaces) with a different left-moving GSO. In other words, a $\operatorname{Spin}(32) / \mathbb{Z}_{2}$ compactification on such a gerbe is equivalent to an $E_{8} \times E_{8}$ compactification on a space. Compactifications of this form are described in Section 4 .

- We conjecture when the bundle is nontrivial over the gerbe, but not rank 8 or the gerbe is not $\mathbb{Z}_{2}$, a perturbative heterotic string compactification is not consistent. That said, we do provide some seemingly consistent $(0,2)$ SCFT's defined by gerbes and bundles of this form,

4 Another thrust of the same papers is a modern discussion of Fayet-Iliopoulos parameters in supergravity - it is argued that they can exist and are quantized. See e.g. 35. for an excellent discussion of old lore on the subject, which is circumvented in the works above. 
but unfortunately they do not seem to be useful for heterotic string compactification. Compactifications of this form are discussed in Section 5 .

In addition, it is also possible to build examples displaying combinations of these classes, which are discussed in Section 6.

In Appendix A we describe how to compute massless spectra in heterotic string compactifications on general stacks. Along the way, we derive some new necessary conditions for well-definedness of a SCFT associated to a heterotic string on a stack, generalizing old statements that " $c_{1} \equiv 0 \bmod 2$ " for a consistent heterotic compactification. Appendix B describes in some depth line bundles on gerbes over projective spaces, as a good prototype for other bundles on more general gerbes. Appendix C discusses how Chern classes and characters are defined for stacks, and in particular, discusses $c^{\text {rep }}$ and $c^{\text {rep}}$, versions of Chern classes and characters which encode information about twisted sectors and which play a vital role in index theory. Appendix D contains a short discussion of roots of canonical bundles on gerbes, a technical matter that sometimes arises in computations.

One of the original motivations of this work was the hope that the third class above would yield new consistent heterotic string compactifications and new consistent $(0,2)$ SCFT's. Although it seems there are new consistent $(0,2)$ SCFT's, we will argue that they do not seem to define new consistent supersymmetric heterotic string compactifications.

In hindsight, we can understand that result as follows. In an ineffective orbifold (one in which part of the orbifold group acts trivially on the space), the twisted sectors contain massless states whose wavefunctions have support over the entire space. This would seem to imply that there are 'extra' ten-dimensional massless states, but this would be a contradiction, since the ten-dimensional supergravity theory is known and fixed. Furthermore, so long as we work at low energies and close to large-radius limits, a tendimensional supergravity analysis should be applicable.

In type II strings, this conundrum was implicity solved by the decomposition conjecture [17]: strings on gerbes are the same as strings on disjoint unions of spaces. The 'extra' states are there, but simply fill out copies of the supergravity theory.

In heterotic strings, we will see a mix of several solutions: in some cases, an analogue of the decomposition conjecture exists; in other cases, the theory is dual to a compactification on a manifold; in yet other cases, the compactification does not seem to be consistent.

${ }^{5}$ We would like to thank J. Gray for pointing this out to us. 


\section{Generalities}

\subsection{Strings on stacks}

Stacks are a form of 'generalized spaces' admitting smooth structures, metrics, bundles, and other structures needed to define sigma models. In particular, stacks are defined by the incoming maps from other spaces, making them a natural setting for defining sigma models.

Stacks have been discussed as target 'spaces' for nonlinear sigma models in a number of references, including 6 [13 23] for two-dimensional $(2,2)$ supersymmetric and [37, 39] for four-dimensional $\mathcal{N}=1$ supersymmetric sigma models. References for physicists on the mathematics of stacks are, unfortunately, somewhat harder to locate. In the mathematics literature, standard references on algebraic stacks include [48 50] and good references on topological stacks include [51 58. In addition, we have striven to write our own papers to be reasonably self-contained (see for example [15] for more information, oriented towards physicsts).

We can make more concrete sense of strings on stacks as follows. Every $]^{7}$ smooth (Deligne-Mumford) stack $\mathfrak{X}$ has a presentation of the form of a global quotient $[X / G]$, where $X$ is a smooth manifold and $G$ is a group which need neither be finite nor act effectively. To such a presentation, we associate a $G$-gauged nonlinear sigma model on $X$.

Now, such presentations are not unique: a given stack can have many presentations of this $\$$ form. In two dimensional $(2,2)$ theories, it is believed, and has been extensively checked, that renormalization group flow 'washes out' such presentation dependence, so universality classes depend only upon the stack, not any particular presentation. Thus, one can meaningfully associate a two-dimensional CFT to a stack, not merely a presentation thereof. In four dimensions, by contrast, this is not believed to be the case. For example, although gauge couplings are dynamically generated in two dimensions, they are not dynamically generated in four dimensions, and the stack does not

\footnotetext{
${ }^{6}$ In addition to the references above on the physics of nonlinear sigma models, there is also an extensive discussion of Gromov-Witten invariants of stacks in the math literature, see for example [44 47] for a few representative examples.

${ }^{7}$ With minor caveats, as discussed in e.g. 15 .

${ }^{8}$ In addition, stacks can have presentations of other forms. However, realizing other types of presentations in physics would require a significant generalization of Faddeev-Popov and Batalin-Vilkovisky gauge-fixing procedures, which we do not claim to understand, so we do not claim that physics can be associated to all presentations.
} 
determine a gauge coupling. Thus, in four dimensions we can not uniquely associate physics to stacks, though we can certainly do the converse, and use stacks to understand some parts of the physics of four-dimensional gauge theories, as in [39].

This paper is concerned with issues around perturbative heterotic strings on stacks, i.e. $(0,2)$ SCFT's. In principle, a perturbative heterotic string will be defined by a Calabi-Yau stack $\mathfrak{X}$ together with a gauge bundle $\mathcal{E}$ over the stack, satisfying certain anomaly cancellation conditions. We understand $(0,2)$ SCFT's in the same fashion as above: we pick a presentation of the stack of the form $[X / G]$. Given such a presentation, the gauge bundle is then a $G$-equivariant bundle $\mathcal{E}$ over $X$. To this data, we associate a $G$ gauged heterotic sigma model on $X$ with gauge bundle $\mathcal{E}$. As before, there can be multiple presentations of a stack with different UV physics, so we conjecture that renormalization group flow washes out such presentationdependence, and only associate universality classes of renormalization group flow to stacks.

Not every $(X, \mathcal{E}, G)$ will define a consistent heterotic string theory; for example, the data above must satisfy anomaly cancellation. One part of anomaly cancellation is clear: before gauging, the heterotic sigma model on $X$ with bundle $\mathcal{E}$ must be anomaly-free, meaning that $\operatorname{ch}_{2}(T X)=\operatorname{ch}_{2}(\mathcal{E})$.

Demanding that the gauge theory be anomaly-free can impose further constraints. One well-known example is level-matching. As discussed in e.g. [59], for orbifolds, level-matching is believed to be equivalent to matching of second Chern classes in equivariant cohomology. (In particular, equivariant Chern classes can be defined intrinsically on the stack, they are independent of the choice of presentation and descend to well-defined objects on the stack.) Equivariant cohomology can be defined on stacks, and in fact forms the 'naive' cohomology theory of a stack. (See Appendix C for more subtle notions.)

However, level-matching (in the form described in [59]) is not sufficient to guarantee that a given theory is consistent [60, 61], and we shall see explicit examples later in Section 5. In Appendix A.4, we discuss another set of consistency conditions that arise, essentially a generalization of the statement that " $c_{1} \equiv 0 \bmod 2$." Specifically, these conditions state that on each component $\alpha$ of the inertia stack, the $\langle\alpha\rangle$-equivariant line bundle

$$
K_{\alpha} \otimes \operatorname{det} \mathcal{E}_{0}^{\alpha}
$$

admit a square root. We defer further discussion of this condition to Appen$\operatorname{dix}$ A.4. 
One of the original goals of this project was to find a suitable generalization of anomaly cancellation, a set of sufficient conditions, valid for arbitrary stacks, that would guarantee that the resulting $G$-gauged heterotic sigma model is consistent, but we have been unable to do this. Instead, we only have the necessary conditions above. We leave the problem of finding sufficient conditions for future work.

The most interesting examples of heterotic strings on stacks are the special case of strings on gerbes. In previous work [17], it was argued that $(2,2)$ supersymmetric strings on gerbes are equivalent to strings on disjoint unions of spaces. For the heterotic string, we shall argue that such a decomposition only exists in general if the gauge bundle is a pullback from a bundle on the base space. More general, 'twisted' bundles exist, and at least sometimes can appear in heterotic compactifications. In fact, it was one of the original goals of this work to construct new $(0,2)$ SCFT's by using twisted bundles, though as we shall argue later, that does not seem to be the case.

In any event, most of this paper will focus on the special case of heterotic strings on gerbes, so in the remainder of this section we shall review some pertinent facts.

\subsection{Review of gerbes}

So far we have realized heterotic strings on stacks as gauged nonlinear sigma models. The special case of gerbes is realized when a subgroup of the gauge group acts trivially on the target space. In this case, even though part of the gauge group acts trivially on the target, it need not act trivially on the gauge bundle, and this will be responsible for 'twisted' bundles.

For purposes of disambiguation, let us distinguish our usage of the term from other appearances in the literature. In some papers, gerbes are used formally to describe characteristic classes of $B$ fields, just as principal bundles can be used to describe characteristic classes of gauge fields, and sometimes they are used in that sense to help characterize nontrivial $B$ fields.

However, our usage in this paper is different. We are not using the term 'gerbe' to describe characteristic classes; instead, we are thinking of gerbes as analogues of spaces on which strings propagate, just as strings can propagate on the total space of a principal bundle.

Let us now turn to reviewing gerbes. We review some basics here, see [17] for another pertinent general description. In general, to specify a $G$-gerbe over a space $X$, given an open cover $\left\{U_{i}\right\}$ of $X$, one specifies $g_{i j k} \in G$ on 
triple overlaps and $\varphi_{i j} \in \operatorname{Aut}(G)$ on double overlaps, obeying the constraints

$$
\varphi_{j k} \circ \varphi_{i j}=\operatorname{Ad}\left(g_{i j k}\right) \circ \varphi_{i k}
$$

on triple overlaps and

$$
g_{j k \ell} g_{i j \ell}=\varphi_{k \ell}\left(g_{i j k}\right) g_{i k \ell} .
$$

on quadruple overlaps. If we let $\operatorname{Out}(G)$ denote the quotient of the group of all automorphisms of $G$ by inner automorphisms, then the $\varphi_{i j}$ above descend to define a principal $\operatorname{Out}(G)$ bundle. If that bundle is trivializable, then we say the gerbe is banded. In this case, the gerbe is effectively specified just by the $g_{i j k}$ 's, which define a characteristic class in $H^{2}(X, Z(G)$ ). (For example, these were the gerbes described in [62.) The more general case, in which the $\operatorname{Out}(G)$ bundle is nontrivial, is known simply as non-banded.

In terms of stacks, a stack $[X / G]$ will be a $(K$-)gerbe if a nontrivial subgroup (denoted $K$ ) of $G$ acts trivially on $X$, by which we mean $g \cdot x=x$ for all $x \in X$ and all $g \in K \subseteq G$. (This is known as an non-effective group action.) Although quotient spaces cannot detect trivial group actions, quotient stacks can, and moreover, so too can the physics 9 of gauge theories. Although such trivial group actions are invisible perturbatively, they show up nonperturbatively, as has been discussed extensively in e.g. [14 17].

As the physics of strings on gerbes will be important in this paper, let us briefly review how nonperturbative physics can detect trivial group actions.

One short answer is that working with a gauge theory containing a noneffective group action is equivalent to restricting the allowed nonperturbative sector: 10 . For example, consider the $\mathbb{P}^{n}$ model, described as a supersymmetric $U(1)$ gauge theory with $n+1$ chiral superfields of charge 1 , but let us instead give the fields charge $k$ instead of charge 1 . Mathematically, this means that a $\mathbb{Z}_{k}$ subgroup of $U(1)$ acts trivially on the chiral superfields, and describes the weighted projective stack $\mathbb{P}_{[k, k, \ldots, k]}^{n}$, which is a $\mathbb{Z}_{k}$ gerbe on $\mathbb{P}^{n}$. Physically, it is straightforward to see that the instantons in this GLSM are the same as the instantons of degree divisible by $k$ in the original $\mathbb{P}^{n}$

\footnotetext{
${ }^{9}$ Historically, this was one of several confusing points in understanding whether strings could be consistently defined on stacks.

${ }^{10}$ Restricting the allowed instanton sectors ordinarily breaks cluster decomposition, and understanding how this can be consistent was, historically, another confusing issue that had to be straightened out to make sense of strings on stacks. Briefly, the answer is that the theory decomposes into a union of theories on ordinary spaces, see e.g. [17, 18, 39] for discussions in two and four-dimensional theories. We will return to this in Section 4
} 
model. As a practical matter, this means that the $U(1)_{A}$ symmetry is broken to $\mathbb{Z}_{2 k(n+1)}$ rather than $\mathbb{Z}_{2(n+1)}$, for example, and also changes correlation functions and quantum cohomology rings.

More globally, if the worldsheet is compact, then the proper definition of the 'charge' of a field is in terms of what bundle it couples to. Changing the bundle changes the allowed zero modes, hence changes anomalies and correlation functions [14. For a noncompact worldsheet, an analogous result can be obtained in two dimensions utilizing theta angles. We distinguish 'gerbe' cases from 'non-gerbe' cases by adding massive minimally-charged fields. The existence of such fields can be sensed, even if their masses are above the cutoff, by examining the periodicity of the theta angle. Since the theta angle acts as an electric field in two dimensions, if we build a capacitor, then by making the plate separation large, one can excite arbitarily-massive field configurations, hence theta angle periodicity measures existence of massive minimally-charged fields [14, 36, 38. In four dimensions, there are analogous methods, involving for example Reissner-Nordstrom black holes and Hawking radiation [39].

A simple example in toroidal orbifolds may help clarify the discussion. Consider the orbifold $\left[X / D_{4}\right]$, where $D_{4}$ is an eight-element group with a $\mathbb{Z}_{2}$ center, such that $D_{4} / \mathbb{Z}_{2}=\mathbb{Z}_{2} \times \mathbb{Z}_{2}$. Assume the central $\mathbb{Z}_{2}$ acts trivially on $X$. From the general analysis above, one would expect that $\left[X / D_{4}\right] \neq\left[X / \mathbb{Z}_{2} \times \mathbb{Z}_{2}\right]$, i.e. that physics 'sees' the trivially-acting $\mathbb{Z}_{2}$, and that is exactly what happens.

Label the elements of $D_{4}$ by

$$
\{1, z, a, b, a z, b z, a b, b a=a b z\}
$$

where $z$ generates the $\mathbb{Z}_{2}$ center, so that the coset $D_{4} / \mathbb{Z}_{2}$ is given by the images of $1, a, b, a b$, which in the $\left(\mathbb{Z}_{2} \times \mathbb{Z}_{2}\right)$ coset we shall denote $\{1, \bar{a}, \bar{b}, \overline{a b}\}$.

The (string) one-loop partition function of $\left[X / D_{4}\right]$ is obtained by summing over twisted sectors defined by all commuting pairs in $D_{4}$. For example, there are no $(a, a b),(b, a b),(a, b)$ twisted sectors, as those pairs do not commute in $D_{4}$. Now, if we compare the $\mathbb{Z}_{2} \times \mathbb{Z}_{2}$ partition function, although individual twisted sector contributions match (as the $\mathbb{Z}_{2}$ acts trivially), the total number is different. For example, the $\mathbb{Z}_{2} \times \mathbb{Z}_{2}$ contains contributions from $(\bar{a}, \overline{a b}),(\bar{b}, \overline{a b})$ and $(\bar{a}, \bar{b})$ twisted sectors, but there are no corresponding $(a, a b),(b, a b),(a, a b)$ contributions in the $D_{4}$ partition function. Thus, we see the one-loop partition functions of the $D_{4}$ and $\mathbb{Z}_{2} \times \mathbb{Z}_{2}$ partition functions are very different, despite the fact that the theories differ by a trivially-acting gauged $\mathbb{Z}_{2}$. 
In fact, in the example above, one can show that the partition function of the $D_{4}$ orbifold is the same as the partition function of a disjoint union of two $\mathbb{Z}_{2} \times \mathbb{Z}_{2}$ orbifolds, one with and the other without discrete torsion. The one-loop partition function of a disjoint union is the sum of the partition functions of the components, and discrete torsion adds a sign to the $(\bar{a}, \overline{a b})$, $(\bar{b}, \overline{a b})$ and $(\bar{a}, \bar{b})$ sectors, so they cancel out of the partition function for the disjoint union. This is a simple example of the 'decomposition conjecture' we review in Section 3.1 .

\subsection{Notions of twisting}

Now that we have outlined gerbes and demonstrated their physical meaningfulness, let us turn to possible bundles over gerbes. A gerbe was defined by a trivial group action on the base space; however, that same group action can be nontrivial on the bundle. The resulting bundle is then interpreted as some sort of twisted bundle, in some sense, as we shall review here.

There are various notions of twisted bundles in the literature. One notion, discussed for example in [63], is of a twisted bundle in which the twisting refers to the fact that the transition functions do not quite close on triple overlaps: instead of

$$
g_{\alpha \beta} g_{\beta \gamma} g_{\gamma \alpha}=1
$$

the transition functions obey

$$
g_{\alpha \beta} g_{\beta \gamma} g_{\gamma \alpha}=h_{\alpha \beta \gamma} I
$$

for some cocycle $h_{\alpha \beta \gamma}$. At the level of the gauge field, such a twisting means that across coordinate patches, the gauge field receives an affine translation in addition to a gauge transformation. Such twisted bundles appear physically on D-branes. After all, under a gauge transformation of the $B$ field, of the form

$$
B \mapsto B+d \Lambda
$$

the Chan-Paton gauge field must necessarily transform as

$$
A \mapsto A-\Lambda
$$

in order to preserve gauge-invariance on the open string worldsheet, and such affine translations correspond, in terms of transition functions, to the modified overlap condition equation (3). However, although such twistings are possible for D-branes, no such twisting is ordinarily possible in heterotic 
strings, because the heterotic gauge field never picks up affine translations across coordinate patches - the heterotic gauge field and the heterotic $B$ field are related in a very different fashion than in D-branes.

A second notion of twisting appears when discussing gerbes. Consider the weighted projective stack $\mathbb{P}_{[k, \ldots, k]}^{N}$, a $\mathbb{Z}_{k}$ gerbe on $\mathbb{P}^{N}$, described physically by an analogue of the supersymmetric $\mathbb{P}^{N}$ model in which chiral superfields have charge $k$ instead of 1 , as discussed earlier. Now, the total space of a line bundle $\mathcal{O}(-n) \rightarrow \mathbb{P}^{N}$ can be described as a quotient of $N+1$ fields $\phi_{i}$ and one field $p$ of charges $1,-n$, respectively. Consider instead a quotient of the fields above in which the $\phi_{i}$ have charge $k$ (and so describe $\mathbb{P}_{[k, \ldots, k]}^{N}$ ), and the field $p$ has charge -1 . This quotient is the total space of a line bundle on the gerbe sometimes denoted $\mathcal{O}(-1 / k)$. (We will discuss line bundles on gerbes in more detail in Appendix $B$.)

We can understand this second notion of twisting in much greater generality, as follows. First, for any stack $\mathfrak{X}$ presented as $\mathfrak{X}=[X / G]$ for some space $X$ and group $G$, a vector bundle (sheaf) on $\mathfrak{X}$ is the same as a $G$-equivariant vector bundle (sheaf) on $X$. Now, suppose that $G$ is an extension

$$
1 \longrightarrow K \longrightarrow G \longrightarrow H \longrightarrow 1
$$

where $K$ acts trivially on $X$, and $G / K \cong H$ acts effectively. In this case, $\mathfrak{X}=[X / G]$ is a $K$-gerbe. A vector bundle on $\mathfrak{X}$ is a $G$-equivariant vector bundle on $X$, and as such, the $K$ action is defined by a representation of $K$ on the fibers of that vector bundle. This is the more general picture of the second notion of twisting. Any bundle on the gerbe that is not a pullback from the base, has a nontrivial action of $K$.

These two notions of twisting are not unrelated. Mathematically, it is a standard result that the category of sheaves on a gerbe decomposes into different sectors containing twisted sheaves on the underlying space, twisted by flat $B$ fields. Moreover, this decomposition is complete: there are no nonzero Ext groups between sheaves in different sectors on the same gerbe. This fact was one of the inspirations for the 'decomposition conjecture' presented in [17], which said that conformal field theories describing strings on gerbes should factorize in the same way, that the CFT's are the same as CFT's on disjoint unions of spaces. The resulting factorization of D-branes reflects the mathematical result above on factorization of sheaves on gerbes.

For completeness, let us discuss this decomposition for the special case of $\mathcal{O}(1 / k) \rightarrow \mathbb{P}_{[k, \ldots, k]}^{N}$. To be twisted in the first sense we discussed, one can show that the rank of the twisted bundle must be divisible by the order of the twisting cocycle's cohomology class. Here, since $\mathcal{O}(1 / k)$ has rank one, the 
order of the cocycle must be one. Indeed, the twistings of $\mathcal{O}(1 / k)$ appearing involve cocycles with trivial cohomology, so there is no rank restriction.

\section{Class I: Gauge bundle a pullback from the base}

We have classified heterotic string compactifications on gerbes into three fundamental classes or 'building blocks' from which more general compactifications can be built. In this and the next two sections, we will examine properties of those classes.

The first class we consider involves the special case that the gauge bundle is a pullback from the base. This is equivalent to the statement that the subgroup $G$ of the gauge group that acts trivially on the base, also acts trivially on the fibers of the gauge bundle.

In this case, we will argue that, at least for banded gerbes, the heterotic $(0,2)$ SCFT factorizes - it is equivalent to a heterotic string on a disjoint union of spaces with bundles, following essentially the same mechanism as in $(2,2)$ strings.

\subsection{Review of $(2,2)$ decomposition conjecture}

As was reviewed earlier in Section 2.2, gauge theories in which a subgroup of the gauge group acts trivially on massless matter break cluster decomposition. However, it was argued in [17] that such theories are equivalent to tensor products / disjoint unions of cluster-decomposition-obeying theories. For example, a gauged nonlinear sigma model of this form is equivalent to a nonlinear sigma model on a disjoint union of ordinary spaces. The latter violates cluster decomposition, but does so in an obviously trivial fashion, and so there is no essential difficulty with the quantum field theory.

For $(2,2)$ supersymmetric gauged nonlinear sigma models in two dimensions, this was encapsulated in [17] in the "decomposition conjecture", which we shall generalize to heterotic strings. To make this paper self-contained, we take a moment here to review the statement of the decomposition conjecture.

Suppose we have a $K$-gerbe over $[X / H]$, defined by the quotient $[X / G]$ where

$$
1 \longrightarrow K \longrightarrow G \longrightarrow H \longrightarrow 1 .
$$

Let $\hat{K}$ denote the set of irreducible representations of $K$. There is a natural action of $H$ on $\hat{K}$, defined as follows: given $h \in H$ and $\rho \in \hat{K}$, pick a lift 
$\tilde{h} \in G$ of $h$, and define $h \cdot \rho$ by,

$$
(h \cdot \rho)(g) \equiv \rho\left(\tilde{h}^{-1} g \tilde{h}\right)
$$

for all $g \in K$. If $K$ is abelian, this is well-defined. If $K$ is not abelian, then it can be shown (see [17] [Section 4]) that there exists an operator intertwining the representations $h \cdot \rho$ defined by any two lifts, hence $h \cdot \rho$ is well-defined in $\hat{K}$.

Then, the decomposition conjecture for $(2,2)$ theories states that a string on the gerbe $[X / G]$ is the same as a string on the disjoint union of spaces $[(X \times \hat{K}) / H]$, together with a flat $B$ field defined in [17] [Section 4].

In the special case that the gerbe $[X / G]$ is banded, the description above simplifies. In this case, the $H$ action on $\hat{K}$ is trivial, and so the decomposition conjecture reduces to the statement that a string on the gerbe $[X / G]$ is the same as a string on a disjoint union of $|\hat{K}|$ copies of $[X / H]$, in which each copy comes with a flat $B$ field determined by acting on the characteristic class of the gerbe with the irreducible representation corresponding to that copy:

$$
\rho \in \hat{K}: H^{2}([X / H], Z(G)) \longrightarrow H^{2}([X / H], U(1)) .
$$

Extensive evidence was presented in [17] for this conjecture, ranging from computations of orbifold spectra and partition functions to GLSM results and quantum cohomology computations. Other results have appeared since. For reasons of brevity, we only list two below:

- This conjecture makes a prediction for Gromov-Witten invariants of stacks, namely that the Gromov-Witten invariants of gerbes are equivalent to Gromov-Witten invariants of disjoint unions of spaces. This was checked in the mathematics literature in e.g. 29 34].

- This conjecture plays an important role in understanding certain GLSM's. Specifically, it was used in [18] to understand LandauGinzburg points of complete intersections of quadrics, resolving some old unanswered questions, and also providing examples of GLSM's that realize geometry in a different way than as a critical locus of a superpotential, that contain non-birational phases, and in some cases, that RG flow to 'noncommutative resolutions' of singular spaces, providing the first physical realizations of those mathematical theories in CFT. The results of [18] have since been checked in e.g. [24, 25] and further examples discussed in [24, 27, 28. The same methods have also been applied to make predictions for Gromov-Witten invariants of noncommutative resolutions in [26]. 
See also the $D_{4}$ orbifold discussed in Section 2.2 for another example.

The result may seem obscure, but there is a simple physical reason for it, namely that in the path integral, summing over the elements of the disjoint union, together with variable $B$ fields, is equivalent to inserting a projection operator that enforces the requirement that only instantons of certain degrees contribute to the theory. Schematically, for a nonlinear sigma model, we can describe the insertion of a projection operator in the form

$$
\int[D \phi] e^{-S}\left(\sum_{k=0}^{n-1} e^{i k \int \phi^{*} \omega}\right)=\sum_{k=0}^{n-1} \int[D \phi] \exp \left(-S+i k \int \phi^{*} \omega\right),
$$

where $\omega$ is the Kähler form on the target space. The left-hand side is the partition function with a projector onto nonperturbative states of certain degrees; the right-hand side is a partition function for a disjoint union of $n$ copies of the original target space, each with a rotated $B$ field, rotated by an amount $k \omega$. Nonbanded gerbes merely represent a more complicated variation.

\subsection{Heterotic decomposition conjecture}

In this section we will describe the heterotic analogue of the decomposition conjecture, for banded gerbes. Briefly, given a $(0,2)$ SCFT defined by a banded gerbe $\mathfrak{X}$ over a space (or orbifold) $X$ and bundle $\mathcal{E} \rightarrow \mathfrak{X}$, such that $\mathcal{E}$ is a pullback of a bundle on $X$, then this $(0,2)$ SCFT is the same as a $(0,2)$ SCFT on a disjoint union of copies of $X$.

Now, let us define terms more precisely. Suppose we have a $K$-gerbe over $[X / H]$, defined by the quotient $\mathfrak{X}=[X / G]$ where

$$
1 \longrightarrow K \longrightarrow G \longrightarrow H \longrightarrow 1 \text {. }
$$

Suppose we also have a holomorphic vector bundle $\mathcal{E}$ over $[X / G]$ (i.e. a $G$-equivariant bundle on $X$ ), defining a consistent $(0,2)$ SCFT.

We assume that $\mathcal{E}$ is a pullback of a bundle $\mathcal{E}^{\prime}$ on $[X / H]$. This can be understood in several equivalent ways, for example:

- $K$ acts trivially on both $X$ and $\mathcal{E}$,

- $\mathcal{E}$ is in the weight-zero part of the decomposition of sheaves on $[X / G]$, which imply that the $G$-equivariant structure on $\mathcal{E}$ (as a bundle on $X$ ) descends to an $H$-equivariant structure. 
The heterotic decomposition conjecture for $(0,2)$ theories is that, in these circumstances, if the gerbe is banded, a heterotic string on $([X / G], \mathcal{E})$ is the same as a heterotic string on the disjoint union

$$
\amalg_{\hat{K}}[X / H]
$$

with varying $B$ fields and gauge bundle $\mathcal{E}^{\prime}$ on each copy of $[X / H]$.

As a consistency check, in the special case that $\mathcal{E}=T \mathfrak{X}$ (i.e. $T X$ with its natural $G$-equivariant structure), then $\mathcal{E}^{\prime}=T X$ with its natural $H$ equivariant structure, and this reduces to the $(2,2)$ decomposition conjecture (for banded gerbes).

Other examples are easy to construct. For example, if we take an anomaly-free heterotic $(0,2)$ SCFT defined by a bundle $\mathcal{E}$ on a space $X$, and take a global orbifold of $X$ by a finite group that acts trivially on both $X$ and $\mathcal{E}$, it is trivial to see that the twisted sector states will all be copies of the untwisted sector states, in agreement with the prediction of the decomposition conjecture above that this $(0,2)$ SCFT should be the same as that for a disjoint union of copies of $(X, \mathcal{E})$.

Another set of examples is provided by $(0,2)$ GLSM's. Begin with an anomaly-free $(0,2)$ GLSM describing a bundle $\mathcal{E}^{\prime}$, say,

$$
0 \longrightarrow \mathcal{E}^{\prime} \longrightarrow \oplus_{a} \mathcal{O}\left(n_{a}\right) \stackrel{F}{\longrightarrow} \oplus_{i} \mathcal{O}\left(m_{i}\right) \longrightarrow 0
$$

over a hypersurface in a weighted projective space $\mathbb{P}_{w_{0}, \ldots, w_{d}}^{d}\left[w_{0}+\cdots+w_{d}\right]$. Now, build a new $(0,2)$ GLSM constructed from the one above by multiplying all gauge charges by an integer $k>0$. The result is a bundle $\mathcal{E}$,

$$
0 \longrightarrow \mathcal{E} \longrightarrow \oplus_{a} \mathcal{O}\left(k n_{a}\right) \stackrel{F}{\longrightarrow} \oplus_{i} \mathcal{O}\left(k m_{i}\right) \longrightarrow 0,
$$

over a hypersurface in a weighted projective stack $\mathbb{P}_{\left[k w_{0}, \ldots, k w_{d}\right]}^{d}\left[k\left(w_{0}+\cdots+\right.\right.$ $\left.w_{d}\right)$ ]. The bundle map $F$ and hypersurface polynomial are unchanged. If one now goes to the Landau-Ginzburg point of this theory and computes the massless spectrum, it is trivial to see that the spectrum will consist of $k$ copies of the spectrum of the original theory, in agreement with the prediction of the decomposition conjecture.

The analogue of the decomposition conjecture for nonbanded gerbes is not currently known. It is tempting to speculate that it should be the statement that a heterotic string on $([X / G], \mathcal{E})$ is the same as a heterotic strings on $([(X \times \hat{K}) / H], \mathcal{E})$, where (as in the $(2,2)$ case) $\hat{K}$ is the set of irreducible representations of $K$, and we extend $\mathcal{E}$ trivially from $[X / H]$ to $[(X \times \hat{K}) / H]$. 
However, on the $(2,2)$ locus, the special case that $\mathcal{E}=T X$ with its natural $G$-equivariant structure, $\mathcal{E}$ reinterpreted as an $H$-equivariant bundle and extended trivially over $\hat{K}$ does not in general ${ }^{11}$ define the tangent bundle of $[(X \times \hat{K}) / H]$, and so this would not reduce correctly to $(2,2)$ decomposition.

\section{Class II: Dualities}

\subsection{Basic proposal}

In the special case of a heterotic string in which a $\mathbb{Z}_{2}$ that acts nontrivially on the base, acts nontrivially on a rank 8 bundle, that subgroup of the gauge group is locally duplicating the effect of one of the ten-dimensional left-moving GSO projections. If one starts with a $\operatorname{Spin}(32) / \mathbb{Z}_{2}$ string, then the dual looks locally like an $E_{8} \times E_{8}$ string.

In this section, we will describe ${ }^{12}$ a precise duality relating such $\operatorname{Spin}(32) / \mathbb{Z}_{2}$ compactifications to ordinary $E_{8} \times E_{8}$ compactifications, and discuss some examples.

First, let us consider the easiest case. If the $\mathbb{Z}_{2}$ gerbe is trivial, the result is automatic: the worldsheet left-moving GSO projection is duplicated exactly, not just locally. When the gerbe is nontrivial, one must think a little harder to find a precise duality.

We propose ${ }^{13}$ a duality to heterotic $E_{8} \times E_{8}$ strings as follows. To set conventions, suppose our stack $\mathfrak{X}=[X / \tilde{G}]$, where

$$
1 \longrightarrow \mathbb{Z}_{2} \longrightarrow \tilde{G} \longrightarrow G \longrightarrow 1
$$

and $\mathbb{Z}_{2}$ acts trivially on $X$. Suppose furthermore that $\mathcal{E}$ is a bundle on $\mathfrak{X}$, i.e. a $\tilde{G}$-equivariant bundle on $X$, whose embedding into $E_{8}$ is via the standard worldsheet fermionic construction, in which left-moving fermions are in the fundamental representation of the structure group. Suppose that the $\mathbb{Z}_{2}$ acts nontrivially on $\mathcal{E}$.

\footnotetext{
11 Only if $K$ lies in the center of $G$ would the tangent bundle have a trivial extension over $\hat{K}$.

${ }^{12}$ We have not been able to locate this particular duality in the literature, but would not be surprised if it has been discussed somewhere previously, presumably in a different context. The closest of which we are aware is old work on T-duality in toroidally compactified heterotic strings, relating $\operatorname{Spin}(32) / \mathbb{Z}_{2}$ strings and $E_{8} \times E_{8}$ strings after the gauge group has been Higgsed to a common subgroup, see for example 64.

13 We would link to thank J. Distler for suggesting this construction to us.
} 
In general $\mathcal{E}$ will not admit a $G$-equivariant structure. Nevertheless, at least in the special case that the $\mathbb{Z}_{2}$ is central in $\tilde{G}$, the bundles $\mathcal{E}^{*} \otimes \mathcal{E}$ and $\wedge^{\text {even }} \mathcal{E}$ will admit a $G$-equivariant structure, and so can be defined on $[X / G]$. Moreover, it was observed in 65] that, for the 'typical' worldsheet embeddings of $S U(n)$ in $E_{8}$ (including the present one), massless spectra of heterotic compactifications on smooth spaces can be defined solely in terms of sheaf cohomology with coefficients in $\mathcal{E}^{*} \otimes \mathcal{E}$ and $\wedge^{\text {even }} \mathcal{E}$; other sheaf cohomology groups are related by Serre duality. There is a good reason for this. In the heterotic compactifications discussed in [65], the $S U(n)$ gauge bundle is embedded into $E_{8}$ by first embedding in $\operatorname{Spin}(2 n) \subset \operatorname{Spin}(16)$, projecting to $\operatorname{Spin}(16) / \mathbb{Z}_{2}$ (as a result of the left GSO projection), and then $\operatorname{Spin}(16) / \mathbb{Z}_{2}$ naturally embeds into $E_{8}$ [66]. The only coefficient bundles that survive the

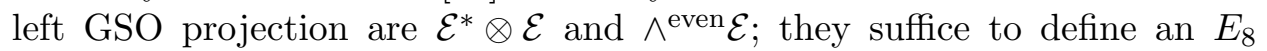
bundle, and that is why they suffice to define massless spectra.

Thus, we propose that a heterotic $\operatorname{Spin}(32) / \mathbb{Z}_{2}$ string compactified on a $\mathbb{Z}_{2}$ gerbe $\mathfrak{X}$ as above, with the $\mathbb{Z}_{2}$ central, acting by signs on a rank 8 bundle $\mathcal{E} \rightarrow \mathfrak{X}$, embedded in a typical fashion, defines the same SCFT as a heterotic $E_{8} \times E_{8}$ string compactified on $[X / G]$ with $E_{8}$ gauge bundle determined by $\mathcal{E}^{*} \otimes \mathcal{E}$ and $\wedge^{\text {even } \mathcal{E}}$ (which are defined on $[X / G]$, even if $\mathcal{E}$ itself is not).

In the special case that the $\mathbb{Z}_{2}$ gerbe is trivial, the dual $E_{8} \times E_{8}$ string on $[X / G]$ is defined by the bundle $\mathcal{E}$ - in this special case, the $E_{8}$ bundle determined by $\mathcal{E}^{*} \otimes \mathcal{E}, \wedge^{\text {even } \mathcal{E}}$ is the same determined by the usual embedding of $\mathcal{E}$ into $E_{8}$. More generally, the $E_{8}$ bundle need not have a description in terms of a similarly-embedded $S U(n)$ gauge bundle; a direct construction might have to appeal to the fibered WZW methods discussed in 67-72].

So far we have discussed $\operatorname{Spin}(32) / \mathbb{Z}_{2}$ compactifications on a $\mathbb{Z}_{2}$ gerbe with rank 8 bundle. Now let us briefly consider an $E_{8} \times E_{8}$ compactification on a $\mathbb{Z}_{2}$ gerbe with rank 8 bundle. Nearly the same analysis applies as in the $\operatorname{Spin}(32) / \mathbb{Z}_{2}$ case. At the level of SCFT, before imposing the left GSO projections, the same duality argument we have just given suggests the gerbe theory should be dual to an $E_{8}$ bundle, as above. The left GSO for the corresponding bundle duplicates the gerbe $\mathbb{Z}_{2}$, and so should act trivially on the theory. The dual should be thus be interpreted as class I, and so the result should have the form of a disjoint union of two copies of an $E_{8} \times E_{8}$ compactification. As the details are largely duplicative of the $\operatorname{Spin}(32) / \mathbb{Z}_{2}$ case just discussed, and for which we will see examples below, we will not treat this case further.

We have discussed bundles with structure group $S U(n)$ embedded into $\operatorname{Spin}(32) / \mathbb{Z}_{2}$ and $E_{8} \times E_{8}$ in the form of the standard worldsheet construction, but more general embeddings exist, and admit worldsheet descriptions 
[67. One open question we leave for future work is to generalize the duality discussed here to more general embeddings.

\subsection{Toroidal orbifold example}

Consider a $\operatorname{Spin}(32) / \mathbb{Z}_{2}$ heterotic string compactified on a $\mathbb{Z}_{2}$ gerbe over $\left[T^{4} / \mathbb{Z}_{2}\right]$, with a rank eight bundle, defined as follows. The $\mathbb{Z}_{2}$ gerbe is $\left[T^{4} / \mathbb{Z}_{4}\right]$, where the $\mathbb{Z}_{4}$ acts on the $T^{4}$ by

$$
x \mapsto \exp \left(\frac{2 \pi i(2 k) k}{4}\right) x=(-)^{k} x,
$$

so that there is a trivially-acting $\mathbb{Z}_{2}$ subgroup; only the sectors $k=1,3$ have twisted bosons. (Mathematically, this is a nontrivia ${ }^{14} \mathbb{Z}_{2}$ gerbe.) The bundle is the rank eight bundle $\mathcal{O}^{\oplus 8}$, on which the $\mathbb{Z}_{4}$ acts (effectively) by fourth roots of unity.

${ }^{14}$ This gerbe is the obstruction to lifting the principal $\mathbb{Z}_{2}$ bundle $T^{4} \rightarrow\left[T^{4} / \mathbb{Z}_{2}\right]$ to a principal $\mathbb{Z}_{4}$ bundle on $\left[T^{4} / \mathbb{Z}_{2}\right]$. But a principal $\mathbb{Z}_{k}$ bundle on any space $X$ is the same thing as a homomorphism $\pi_{1}(X) \rightarrow \mathbb{Z}_{k}$. Therefore, we can study nontriviality of the gerbe as a question about lifts of group homomorphisms. The bundle $T^{4} \rightarrow\left[T^{4} / \mathbb{Z}_{2}\right]$ corresponds to a homomorphism

$$
\phi: \pi_{1}\left(\left[T^{4} / \mathbb{Z}_{2}\right]\right) \longrightarrow \mathbb{Z}_{2}
$$

(In particular, since $T^{4} \rightarrow\left[T^{4} / \mathbb{Z}_{2}\right]$ is a principal $\mathbb{Z}_{2}$ bundle, we have a long exact sequence with relevant part

$$
\pi_{1}\left(T^{4}\right) \longrightarrow \pi_{1}\left(\left[T^{4} / \mathbb{Z}_{2}\right) \stackrel{\phi}{\longrightarrow} \pi_{0}\left(\mathbb{Z}_{2}\right)\left(\cong \mathbb{Z}_{2}\right) \longrightarrow \pi_{0}\left(T^{4}\right),\right.
$$

and as $T^{4}$ is connected, we see that $\phi$ is surjective.) We want to understand whether $\phi$ lifts to a homomorphism

$$
\psi: \pi_{1}\left(\left[T^{4} / \mathbb{Z}_{2}\right]\right) \longrightarrow \mathbb{Z}_{4}
$$

First note

$$
\pi_{1}\left(\left[T^{4} / \mathbb{Z}_{2}\right]\right)=\mathbb{Z}_{2} \rtimes \mathbb{Z}^{4}
$$

where the nontrivial element in $\mathbb{Z}_{2}$ acts as multiplication by -1 on $\mathbb{Z}^{4}$. The homomorphism $\phi$ is the projection to $\mathbb{Z}_{2}$. The maximal 2-group quotient of $\mathbb{Z}_{2} \rtimes \mathbb{Z}^{4}$ is $\mathbb{Z}_{2} \times\left(\mathbb{Z}_{2}\right)^{4}$, so any homomorphism $\mathbb{Z}_{2} \rtimes \mathbb{Z}^{8} \rightarrow \mathbb{Z}_{4}$ will factor through $\mathbb{Z}_{2} \times\left(\mathbb{Z}_{2}\right)^{4}$. But in the map $\mathbb{Z}_{4} \rightarrow \mathbb{Z}_{2}$, the generator of $\mathbb{Z}_{4}$ maps onto the generator of $\mathbb{Z}_{2}$. Since $\mathbb{Z}_{2} \times\left(\mathbb{Z}_{2}\right)^{4}$ does not contain any element of order 4 , there is no map $\mathbb{Z}_{2} \times\left(\mathbb{Z}_{2}\right)^{4} \rightarrow \mathbb{Z}_{4}$ that lifts the projection onto the first factor. Therefore, the $\mathbb{Z}_{2}$ gerbe is nontrivial. More generally, if $\left[T^{4} / \mathbb{Z}_{2 k}\right]$ is a $\mathbb{Z}_{k}$ gerbe over $\mathbb{Z}_{2}$, where the $Z_{2 k}$ acts by first projecting to $\mathbb{Z}_{2}$, then it is nontrivial. 
We will compute the spectrum, and discover not only that it is consistent, but in addition that it has the same form as the spectrum of a perturbative $E_{8} \times E_{8}$ compactification on a space, as expected from the duality proposal.

We use $X^{3-4}$ to denote the bosons in the $T^{4}$, and $\psi^{3-4}$ their right-moving superpartners. We shall use $\lambda^{1-8}$ to denote the free left-moving fermions and $\lambda^{9-16}$ to denote the left-moving fermions in the bundle above.

Let us begin the spectrum computation in the untwisted sector.

First, consider (NS, NS) states. Here, the left- and right-moving vacuum energies are given by $E_{\text {left }}=-1, E_{\text {right }}=-1 / 2$. The $\mathbb{Z}_{4}$-invariant states have the form

\begin{tabular}{cc} 
State & Count \\
\hline$\left(\lambda_{-1 / 2}^{1-8}, \bar{\lambda}_{-1 / 2}^{1-8}\right)^{2} \otimes\left(\psi_{-1 / 2}^{1-2}, \bar{\psi}_{-1 / 2}^{1-2}\right)$ & spacetime vector, valued in adjoint of $s o(16)$ \\
$\bar{\partial} X_{-1}^{1-2} \otimes\left(\psi_{-1 / 2}^{1-2}, \bar{\psi}_{-1 / 2}^{1-2}\right)$ & gravity, tensor multiplet contribution \\
$\left(\lambda_{-1 / 2}^{9-16} \bar{\lambda}_{-1 / 2}^{9-16}\right) \otimes\left(\psi_{-1 / 2}^{1-2}, \bar{\psi}_{-1 / 2}^{1-2}\right)$ & $(\mathbf{1}$ from the trace) \\
$\bar{\partial} X_{-1}^{3-4} \otimes\left(\psi_{-1 / 2}^{3-4}, \bar{\psi}_{-1 / 2}^{3-4}\right)$ & spacetime vector, valued in adjoint, $\mathbf{1}$ of $s u(8)$ \\
$\left(\left(\lambda_{-1 / 2}^{9-16}\right)^{2},\left(\bar{\lambda}_{-1 / 2}^{9-16}\right)^{2}\right) \otimes\left(\psi_{-1 / 2}^{3-4}, \bar{\psi}_{-1 / 2}^{3-4}\right)$ & $\begin{array}{c}\text { spacetime scalars (toroidal moduli), } \\
\text { forming } 4 \text { hypermultiplets }\end{array}$ \\
4 spacetime scalars, \\
valued in $\wedge^{2} \mathbf{8}=\mathbf{2 8}, \wedge^{2} \overline{\mathbf{8}}=\overline{\mathbf{2 8}}$ of $s u(8)$, \\
forming 1 hypermultiplet in $\mathbf{2 8}$, \\
another in $\overline{\mathbf{2 8}}$
\end{tabular}

There are no $(\mathrm{R}, \mathrm{NS})$ states in the untwisted sector, since $E_{\text {left }}>0$.

Next, consider the twisted sector $k=1$.

In the (NS,NS) sector, fields have the following boundary conditions:

$$
\begin{aligned}
X^{1-2}(\sigma+2 \pi) & =+X^{1-2}(\sigma), \\
X^{3-4}(\sigma+2 \pi) & =-X^{3-4}(\sigma) \\
\psi^{1-2}(\sigma+2 \pi) & =-\psi^{1-2}(\sigma) \\
\psi^{3-4}(\sigma+2 \pi) & =+\psi^{3-4}(\sigma) \\
\lambda^{1-8}(\sigma+2 \pi) & =-\lambda^{1-8}(\sigma) \\
\lambda^{9-16}(\sigma+2 \pi) & =-\exp \left(\frac{2 \pi i}{4}\right) \lambda^{9-16}(\sigma) .
\end{aligned}
$$

It is straightforward to compute $E_{\text {left }}=-1 / 2, E_{\text {right }}=0$. The available field modes are

$$
X_{-1 / 2}^{3-4}, \quad \lambda_{-1 / 2}^{1-8}, \bar{\lambda}_{-1 / 2}^{1-8}, \quad \lambda_{-1 / 4}^{9-16}, \bar{\lambda}_{-3 / 4}^{9-16}
$$


There is a multiplicity of right-moving Fock vacua, arising from the periodicity of $\psi^{3-4}$. Briefly, the vacua $| \pm \mp\rangle$ are invariant, and $| \pm \pm\rangle$ get a sign under the action of the generator of $\mathbb{Z}_{4}$.

In this sector, the $\mathbb{Z}_{4^{-}}$and GSO-invariant states are

\begin{tabular}{cc} 
State & Count \\
\hline $\bar{\partial} X_{-1 / 2}^{3-4} \otimes| \pm \pm\rangle$ & 8 spacetime scalars \\
$\left(\lambda_{-1 / 4}^{9-16}\right)^{2} \otimes| \pm \pm\rangle$ & 2 spacetime scalars valued in $\wedge^{2} \mathbf{8}=\mathbf{2 8}$ of $s u(8)$
\end{tabular}

There are no massless states in (R, NS) in this sector, as $E_{\text {left }}=+1 / 2$.

Copies of the states in the $k=1$ sector occur at each of the sixteen fixed points, hence the total state count should be obtained by multiplying the totals for this sector by sixteen.

Next, consider the twisted sector $k=2$.

In the (NS, NS) sector, fields have the following boundary conditions:

$$
\begin{aligned}
X^{1-4}(\sigma+2 \pi) & =+X^{1-4}(\sigma), \\
\psi^{1-4}(\sigma+2 \pi) & =-\psi^{1-4}(\sigma), \\
\lambda^{1-8}(\sigma+2 \pi) & =-\lambda^{1-8}(\sigma), \\
\lambda^{9-16}(\sigma+2 \pi) & =+\lambda^{9-16}(\sigma) .
\end{aligned}
$$

It is straightforward to compute $E_{\text {left }}=0, E_{\text {right }}=-1 / 2$. The available field modes are

$$
\psi_{-1 / 2}^{\mu}, \bar{\psi}_{-1 / 2}^{\mu}, \lambda_{-1 / 2}^{1-8}, \bar{\lambda}_{-1 / 2}^{1-8}
$$

There is a multiplicity of left Fock vacua, arising from $\lambda^{9-16}$. Let $|m, n\rangle$ denote a vacuum with $m+$ 's and $n$-'s (note $m+n=8$ ), then under the action of the generator of $\mathbb{Z}_{4}$, it is straightforward to check that $|m=0,4,8\rangle$ are invariant, $|m=2,6\rangle$ get a sign flip, and the others get other fourth roots of unity.

The $\mathbb{Z}_{4^{-}}$and GSO-invariant states in this sector are of the form

$$
\begin{array}{cc}
\text { State } & \text { Count } \\
\hline|m=0,4,8\rangle \otimes\left(\psi_{-1 / 2}^{1-2}, \bar{\psi}_{-1 / 2}^{1-2}\right) & \text { spacetime vectors, in the } \mathbf{1}, \mathbf{1}, \wedge^{4} \mathbf{8}=\mathbf{7 0} \text { of } s u(8) \\
|m=2,6\rangle \otimes\left(\psi_{-1 / 2}^{3-4}, \bar{\psi}_{-1 / 2}^{3-4}\right) & 1 \text { hypermultiplet in } \wedge^{2} \mathbf{8}=\mathbf{2 8}, \wedge^{2} \overline{\mathbf{8}}=\overline{\mathbf{2 8}} \text { of } s u(8)
\end{array}
$$


The (R, NS) sector in $k=2$ is closely related. Here, fields have the following boundary conditions:

$$
\begin{gathered}
X^{1-4}(\sigma+2 \pi)=+X^{1-4}(\sigma), \\
\psi^{1-4}(\sigma+2 \pi)=-\psi^{1-4}(\sigma), \\
\lambda^{1-8}(\sigma+2 \pi)=+\lambda^{1-8}(\sigma), \\
\lambda^{9-16}(\sigma+2 \pi)=-\lambda^{9-16}(\sigma) .
\end{gathered}
$$

Just as in the (NS, NS) sector, $E_{\text {left }}=0$ and $E_{\text {right }}=-1 / 2$. Here, the left Fock vacua form a spinor of the low-energy so(16).

The $\mathbb{Z}_{4}$-invariant states in this sector are of the form

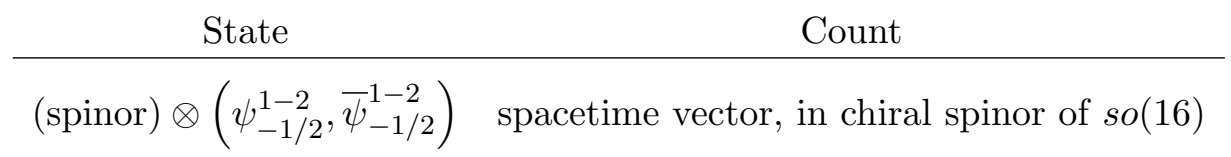

Finally, let us consider the $k=3$ sector. There are no massless states in (R, NS), so we only consider (NS, NS). Fields in this sector have the following boundary conditions:

$$
\begin{aligned}
X^{1-2}(\sigma+2 \pi) & =+X^{1-2}(\sigma), \\
X^{3-4}(\sigma+2 \pi) & =-X^{3-4}(\sigma), \\
\psi^{1-2}(\sigma+2 \pi) & =-\psi^{1-2}(\sigma), \\
\psi^{3-4}(\sigma+2 \pi) & =+\psi^{3-4}(\sigma), \\
\lambda^{1-8}(\sigma+2 \pi) & =-\lambda^{1-8}(\sigma), \\
\lambda^{9-16}(\sigma+2 \pi) & =\exp \left(\frac{\pi i}{2}\right) \lambda^{9-16}(\sigma) .
\end{aligned}
$$

It is straightforward to compute $E_{\text {left }}=-1 / 2, E_{\text {right }}=0$. The available field modes are

$$
\bar{\partial} X_{-1 / 2}^{3-4}, \quad \lambda_{-1 / 2}^{1-8}, \bar{\lambda}_{-1 / 2}^{1-8}, \quad \lambda_{-3 / 4}^{9-16}, \bar{\lambda}_{-1 / 4}^{9-16}
$$

Because $\psi^{3-4}$ is periodic, there is a multiplicity of right Fock vacua. The states $|+-\rangle,|-+\rangle$ are invariant under the generator of $\mathbb{Z}_{4}$, whereas the states $|++\rangle,|--\rangle$ get a sign flip. 
Putting this together, we find $\mathbb{Z}_{4^{-}}$and GSO-invariant massless states of the form:

\begin{tabular}{cc} 
State & Count \\
\hline$\left(\bar{\partial} X_{-1 / 2}^{3-4}, \bar{\partial}_{-1 / 2}^{3-4}\right) \otimes| \pm \pm\rangle$ & 8 scalars \\
$\left(\bar{\lambda}_{-1 / 4}^{9-16}\right)^{2} \otimes| \pm \pm\rangle$ & 2 sets of scalars each in the $\wedge^{2} \overline{\mathbf{8}}=\overline{\mathbf{2 8}}$ of $s u(8)$
\end{tabular}

Furthermore, copies of the states above occur at each fixed point, hence the total number of states is obtained by multiplying the tally above by sixteen.

Now, let us summarize our results so far. We have found the following states:

- 1 gravity multiplet,

- 1 tensor multiplet,

- vector multiplets transforming in the adjoint, chiral spinor of so(16),

- vector multiplets transforming in the adjoint, $\mathbf{7 0}, \mathbf{1}, \mathbf{1}, \mathbf{1}$ of $s u(8)$,

- 10 hypermultiplets in $\mathbf{2 8}$ of $s u(8)(k=0,1,2)$,

- 10 hypermultiplets in $\overline{\mathbf{2 8}}$ of $s u(8)(k=0,2,3)$,

- $4(k=0)$ plus $32(k=1)$ plus $32(k=3)$ singlet hypermultiplets.

We can describe this spectrum more compactly as follows. First, the vectors transforming in the adjoint and chiral spinor of $s o(16)$ clearly combine to form a vector in the adjoint of $e_{8}$. Second, under its $s u(8)$ subalgebra, the adjoint representation of $e_{7}$ decomposes as [73] [Table 52]

$$
133=63+70
$$

so we see that the remaining non-singlet vectors combine to form the adjoint of $e_{7}$. In the same decomposition,

$$
56=28+\overline{28}
$$

so we see that the hypermultiplets in the $\mathbf{2 8}$ and $\overline{\mathbf{2 8}}$ combine to form 10 hypermultiplets in the $\mathbf{5 6}$ of $e_{7}$.

Putting this together, we find that the spectrum can be described as follows:

- 1 gravity multiplet, 
- 1 tensor multiplet,

- vector multiplets transforming in the adjoint of $E_{7} \times E_{8} \times U(1)^{3}$,

- 10 hypermultiplets in the $\mathbf{5 6}$ of $E_{7}$,

- 68 hypermultiplets that are singlets under $E_{7} \times E_{8}$.

The number of hypermultiplets is greater than the number of vector multiplets by 244, which is a necessary condition for anomaly cancellation.

The duality proposal in this example predicts that the dual is defined by a heterotic $E_{8} \times E_{8}$ compactification on $\left[T^{4} / \mathbb{Z}_{2}\right]$, with $E_{8}$ bundle defined by $\mathcal{E}^{*} \otimes \mathcal{E}, \wedge^{\text {even }} \mathcal{E}$, for $\mathcal{E}=\mathcal{O}^{8}$ on $T^{4}$, but such that $\mathcal{E}^{*} \otimes \mathcal{E}$ and $\wedge^{\text {even }} \mathcal{E}$ are odd under the action of the $\mathbb{Z}_{2}$ defining $\left[T^{4} / \mathbb{Z}_{2}\right]$. We do not see how such an $E_{8}$ bundle on $\left[T^{4} / \mathbb{Z}_{2}\right]$ could be obtained from embedding an $S U(n)$ bundle in the usual fashion, and indeed, as remarked earlier, it need not be, the duals in general may only be describable by fibered WZW models. That said, the reader should note that the spectrum computed above is nearly the same as the massless spectrum of an $E_{8} \times E_{8}$ string compactified on a $(2,2)\left[T^{4} / \mathbb{Z}_{2}\right]$, which in general terms is consistent with the existence of a duality between the current $\operatorname{Spin}(32) / \mathbb{Z}_{2}$ gerbe compactification and an $E_{8} \times E_{8}$ compactification. So, although we cannot check the details at this time, certainly in broad brushstrokes this is consistent.

\subsection{Examples in Distler-Kachru models}

In Table 1 we tabulate the combinatorial data for a number of anomaly-free Distler-Kachru $(0,2)$ GLSM's of the pertinent form. Each describes a bundle $\mathcal{E}$ over a Calabi-Yau hypersurface in a weighted projective stack,

$$
\mathbb{P}_{\left[w_{0}, \ldots, w_{n}\right]}^{n}\left[w_{0}+\cdots+w_{n}\right]
$$

a $\mathbb{Z}_{2}$ gerbe over a Calabi-Yau space, where the (rank 8) bundle is given as a kernel of the form

$$
0 \longrightarrow \mathcal{E} \longrightarrow \oplus_{a} \mathcal{O}\left(n_{a}\right) \longrightarrow \oplus_{i} \mathcal{O}\left(m_{i}\right) \longrightarrow 0
$$

For example, the first entry in Table 1 describes a rank 8 bundle given as a kernel

$$
0 \longrightarrow \mathcal{E} \longrightarrow \oplus_{1}^{9} \mathcal{O}(1) \longrightarrow \mathcal{O}(9) \longrightarrow 0
$$

over the stack $\mathbb{P}_{[2,2,2,4]}^{3}[10]$, a $\mathbb{Z}_{2}$ gerbe over $\mathbb{P}_{[1,1,1,2]}^{3}[5]$. 


\begin{tabular}{ccc}
$w_{0}, \ldots, w_{4}$ & $n_{a}$ & $m_{i}$ \\
\hline $2,2,2,4$ & $1^{9}$ & 9 \\
$2,2,2,2,2$ & $1^{9}, 9$ & 7,11 \\
$2,2,2,2,2$ & $3^{9}, 19$ & 9,21 \\
$2,2,2,2,4$ & $3^{9}, 27$ & 9,29 \\
$2,2,2,4,6$ & $1^{8}, 5^{2}$ & 9,13 \\
$2,2,2,2,6$ & $1^{9}, 9$ & 3,15 \\
$2,2,2,4,4$ & $1^{9}, 15$ & 5,19
\end{tabular}

Table 1: This table lists combinatorial data for anomaly-free $(0,2)$ GLSM's describing rank 8 bundles over $\mathbb{Z}_{2}$ gerbes on Calabi-Yau's.

We list a few rank 9 examples over $\mathbb{Z}_{3}$ gerbes in Section 5.2. These rank 8 examples are listed in this section because we are enumerating rank 8 bundles over $\mathbb{Z}_{2}$ gerbes, and the rank 9 examples are not candidates for the dualities discussed here.

Curiously, we were unable to find solutions of the combinatorial consistency conditions for GLSM's for bundles of rank less than 8. We do not know whether this reflects a fundamental limitation of GLSM's, or merely the inadequacy of our parameter space search.

Given a Distler-Kachru model with a phase describing a LandauGinzburg model over an orbifold of a vector space, methods exist to compute the massless spectrum in that Landau-Ginzburg phase [74, 75]. When

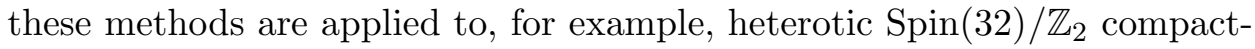
ifications on typical examples from the table above, we find a large number of single vectors and matter representations which likely combine to form representations of a larger nonabelian gauge symmetry, but unfortunately the corresponding worldsheet global symmetry does not seem to be visible in the UV. We conclude that in these examples, much of the needed worldsheet global symmetry appears in the IR, where we have no direct access. This is atypical of Distler-Kachru models, where spacetime gauge symmetries typically appear as worldsheet global symmetries visible in the UV, but is not contradicted by any physics we know. In any event, spectrum computations at Landau-Ginzburg points in these theories have not proven insightful.

\section{Class III: Twisted bundles}

The third fundamental class of examples we shall discuss involve cases in which the trivially-acting part of the gauge group acts nontrivially on the 
bundle, but is not one of the special cases discussed in Section 4 in which the effect is merely to recreate part of the ten-dimensional left-moving GSO projection.

One reason for interest is that examples of this form have the potential to define new heterotic string compactifications. Other reasons also exist, revolving around making sense of heterotic orbifolds with invariant nonequivariant bundles. We review such motivations in Subsection 5.1 .

In Subsection 5.2, we describe some (indirect) constructions of $(0,2)$ SCFT's of this form, via dimensional reduction of consistent four-dimensional theories, and via anomaly-free $(0,2)$ GLSM's.

Unfortunately, although there seem to exist consistent $(0,2)$ SCFT's, they do not seem to yield consistent perturbative heterotic string compactifications. The essential problem is that any finite group that acts only on leftmovers, locally looks like a modification of the ten-dimensional left-moving GSO projection, and as the consistent ten-dimensional GSO projections are already known, if it is not one of them, the results cannot be well-behaved. We will give several examples of six-dimensional compactifications of this form, in which the six dimensional theory has anomalies and cannot be consistent. We outline in detail some examples in which heterotic string compactifications on these $(0,2)$ SCFT's break down in Subsections 5.3, 5.4. and 5.5 .

That said, it may sometimes be possible to restore these theories by adding suitable phases to twisted sectors. For example, the ten-dimensional nonsupersymmetric $S O(8) \times S O(24)$ string seems to be obtainable by a procedure along these lines. Specifically, in the worldsheet theory, if one takes the $\operatorname{Spin}(32) / \mathbb{Z}_{2}$ string and performs an additional left-moving $\mathbb{Z}_{2}$ orbifold on 4 complex fermions, the result satisfies level-matching but does not define a modular-invariant theory; if one then adds phases to restore modular invariance, the result is the nonsupersymmetric ten-dimensional $S O(8) \times S O(24)$ string. (See e.g. [76, 77], 78] [Section 11.3] for more information on this nonsupersymmetric string.) Unfortunately, we do not have a procedure for finding such phases (or even checking whether they exist), and if they do, the previous example suggests that the results will not be supersymmetric. In addition, see e.g. [79, 80, for a different set of ideas which may be relevant, though we have not considered them carefully in this context.

In Subsection 5.6 we outline a few attempts to find a way to understand these issues in terms of some sort of anomaly cancellation. 


\subsection{Motivations}

One reason for interest in this class of examples is that they potentially could describe new $(0,2)$ SCFT's.

Another reason to be interested in them is that they may give a way of understanding heterotic compactifications on ordinary spaces but with non-equivariant bundles. In this section we will explain this motivation.

Let $X$ be a Calabi-Yau manifold, with stable holomorphic vector bundle $\mathcal{E} \rightarrow X$ satisfying anomaly cancellation, so that the pair $(X, \mathcal{E})$ defines a consistent large-radius heterotic Calabi-Yau compactification.

Now, suppose a finite group $G$ acts on $X$. In order to construct a $G$ orbifold of the heterotic string on $(X, \mathcal{E})$, we need for the bundle $\mathcal{E}$ to admit a $G$-equivariantizable structure, which means that for every $g \in G$, we need a lift $\tilde{g}: \mathcal{E} \rightarrow \mathcal{E}$ such that

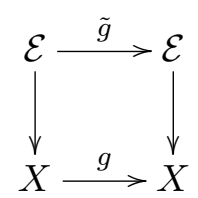

and also such that the lifts obey the group law: $\tilde{g} \circ \tilde{h}=\widetilde{g h}$.

We need such an equivariant structure on the bundle $\mathcal{E}$ for the following two reasons:

- In the worldsheet theory, such an equivariant structure enables us to define a group action on the worldsheet fermions/bosons describing the bundle, such that summing over twisted sectors in the orbifold yields an honest projection operator onto $G$-invariant states.

- In the low-energy supergravity, if $\mathcal{E}$ does not have an equivariant structure, then even if $G$ acts freely, on the quotient $X / G$ the bundle $\mathcal{E}$ will descend to a 'twisted' bundle, not an honest bundle, whose transition functions $g_{\alpha \beta}$ obey

$$
g_{\alpha \beta} g_{\beta \gamma} g_{\gamma \alpha}=h_{\alpha \beta \gamma} I
$$

on triple overlaps, and whose gauge field $A$ obeys

$$
A_{\beta}=g_{\alpha \beta} A_{\alpha} g_{\alpha \beta}^{-1}+g_{\alpha \beta}^{-1} d g_{\alpha \beta}+\Lambda_{\alpha \beta} I
$$

across intersections, for some affine translation $\Lambda_{\alpha \beta}$. As ten-dimensional super-Yang-Mills only describes honest bundles and ordinary gauge 
transformations, the structure above cannot be used to define a consistent string compactification.

However, there is a workaround. If the bundle $\mathcal{E}$ is invariant (meaning, its characteristic classes are invariant under the group action), but not equivariant, then we can find a larger group $\tilde{G}$, an extension of $G$ by a trivially-acting subgroup, such that $\mathcal{E}$ does admit a $\tilde{G}$-equivariant structure, and then take a $G^{\prime}$ orbifold. This is precisely an example of a heterotic string on a gerbe, in this case a gerbe over $[X / G]$.

First, let us review some generalities on the construction of $G^{\prime}$. There is a 'universal', 'maximal' extension $\tilde{G}_{\max }$, which extends $G$ by the group of all automorphisms of the total space of $\mathcal{E}$ that cover the action of the elements of $G$ on $X$. It fits into a short exact sequence

$$
1 \longrightarrow \operatorname{Aut}(\mathcal{E}) \longrightarrow \tilde{G}_{\max } \longrightarrow G \longrightarrow 1
$$

where $\operatorname{Aut}(\mathcal{E})$ is the group of global bundle automorphisms of $\mathcal{E}$. The group we want, $\tilde{G}$, will necessarily be a subgroup of this universal extension of $\tilde{G}_{\max }$.

In general, the extension defining $\tilde{G}_{\max }$ will not be central, but if $\mathcal{E}$ is stable or simple then $\operatorname{Aut}(\mathcal{E})=\mathbb{C}^{\times}$, and the extension is central. The group $\tilde{G}_{\max }$ acts by definition on $\mathcal{E}$ and so defines an equivariant structure. Every other group for which one has an equivariant structure will map to $\tilde{G}_{\max }$ and the equivariant structure will factor through that map.

Now, clearly, $\tilde{G}_{\max }$ is not a finite group, and we only want to consider cases in which the trivially-acting subgroup is finite. If $G$ is finite and $\mathcal{E}$ is stable or simple, then $\tilde{G}_{\max }$ is a central extension of $G$ by $\mathbb{C}^{\times}$and, because

$$
H^{2}\left(G, \mathbb{C}^{*}\right)=H^{2}(G, \mathbb{Q} / \mathbb{Z})
$$

for $G$ finite, the relevant $H^{2}$ is finite and so every extension is induced from some central extension $G_{\text {min }}$ of $G$ by a finite group of order bounded by the maximal order of elements in $G$. In this fashion, we can construct a $\tilde{G}$.

So far, we have described how, given a bundle that is invariant but not equivariant with respect to an orbifold group $G$, one can extend $G$ to a larger finite group $\tilde{G}$, where the extension acts trivially on the base. Now, not any $\tilde{G}$ will be acceptable: the orbifold by $\tilde{G}$ must, at minimum, satisfy levelmatching, and as discussed earlier, even more in order to define a consistent heterotic string compactification.

For completeness, let us now consider some possible examples. 
One example is described in the paper [81]. (See also [82 87].) In that paper, the authors first construct an elliptically-fibered Calabi-Yau threefold $Z$ with fundamental group $\mathbb{Z}_{2} \times \mathbb{Z}_{2}$, built as a freely-acting $15 \mathbb{Z}_{2} \times \mathbb{Z}_{2}$ quotient of a simply-connected Calabi-Yau threefold $X$ :

$$
Z=X /\left(\mathbb{Z}_{2} \times \mathbb{Z}_{2}\right)
$$

together with a bundle $V$ on $X$ that is not quite equivariant with respect to the $\mathbb{Z}_{2} \times \mathbb{Z}_{2}$ action, and so descends to a twisted bundle on $Z$.

Consider the gerbe presented as $[X / G]$, where

$$
1 \longrightarrow \mathbb{Z}_{2} \longrightarrow G \longrightarrow \mathbb{Z}_{2} \times \mathbb{Z}_{2} \longrightarrow 1
$$

with the $\mathbb{Z}_{2}$ kernel acting trivially. (Explicitly, the extension above is the Heisenberg extension, and $G=D_{4}$ [89].) The bundle $V$ above descends to a bundle on a gerbe. Furthermore, the entire bundle is an eigenbundle under the nontrivial element of the center of $D_{4}$, with eigenvalue - 1 (since it must square to the identity and can not itself be the identity) [89].

For completeness, let us now work through the example of [81] in more detail. Their Calabi-Yau manifold $X$ is an elliptic fibration over a rational elliptic surface, and in fact can be described as the fiber product over $\mathbb{P}^{1}$ of two rational elliptic surfaces $B, B^{\prime}$ :

$$
X=B \times_{\mathbb{P}^{1}} B^{\prime}
$$

where $\pi: X \rightarrow B^{\prime}, \pi^{\prime}: X \rightarrow B, \beta^{\prime}: B^{\prime} \rightarrow \mathbb{P}^{1}, \beta: B \rightarrow B$ :

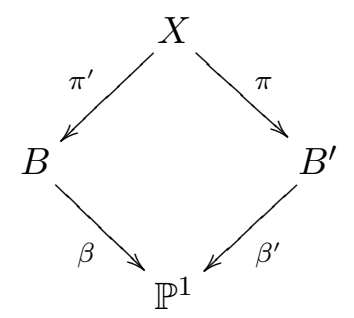

${ }^{15}$ For further examples of Calabi-Yau threefolds with this property, see e.g. 88. Examples include $\mathbb{P}^{7}[2,2,2,2]$ and $\left(\mathbb{P}^{1}\right)^{4}$ with a degree $(2,2,2,2)$ hypersurface. For both, the restriction of an ambient hyperplane class to the Calabi-Yau defines a line bundle which is invariant but not equivariant. 
$B$ and $B^{\prime}$ are both chosen to admit an automorphism group containing $\mathbb{Z}_{2} \times$ $\mathbb{Z}_{2}$. A stable rank four vector bundle $V \rightarrow X$ is constructed as an extension

$$
0 \longrightarrow V_{1} \longrightarrow V \longrightarrow V_{2} \longrightarrow 0,
$$

where

$$
V_{i}=\pi^{\prime *} W_{i} \otimes \pi^{*} L_{i},
$$

for $W_{i}$ a pair of rank 2 vector bundles on $B$ and $L_{i}$ a pair of line bundles on $B^{\prime}$.

Briefly, 81 first argues that each $V_{i}$ is $\mathbb{Z}_{2} \times \mathbb{Z}_{2}$-equivariant. As a result, the group of extensions $\operatorname{Ext}^{1}\left(V_{2}, V_{1}\right)$ decomposes into subspaces associated with characters of $\mathbb{Z}_{2} \times \mathbb{Z}_{2}$. By picking an extension in a subspace associated with the trivial representation, we get a bundle $V$ which is at least $\mathbb{Z}_{2} \times \mathbb{Z}_{2^{-}}$ invariant, though not necessarily $\mathbb{Z}_{2} \times \mathbb{Z}_{2}$-equivariant.

Again, for this example to be physically meaningful, the orbifold group would have to, at minimum, satisfy level-matching. As our purpose in this section was merely to outline one of the motivations for considering heterotic compactifications on gerbes, and we will argue later that these examples are, in most cases, not physically useful, we will end our discussion here.

\subsection{Constructions of consistent CFT's}

In this section, we will describe some constructions of what seem to be consistent $(0,2)$ SCFT's describing heterotic strings on gerbes with fractional gauge bundles. For reasons described elsewhere, these cannot be consistently used in supersymmetric heterotic string compactifications, but nevertheless they do seem to be examples of consistent $(0,2)$ SCFT's.

Our first example was discussed in [90]. Specifically, in [90] [Section 3.1], an $\mathcal{N}=2$ gauge theory in four dimensions with hypermultiplets transforming in the $R$ representation of the gauge group, was reduced along a Riemann surface $C$ to a two-dimensional $(0,4)$ theory, a heterotic nonlinear sigma model whose target is the Hitchin moduli space $\mathcal{M}_{H}(G, C)$ and with a twisted gauge bundle $\mathcal{R}$, defined by the representation $R$ in which the hypermultiplets transform. The four-dimensional theory was partially topologically twisted along a $U(1)_{R}$ (and only exists for superconformal field theories for which that $U(1)_{R}$ is nonanomalous).

In this example, the gauge bundle is twisted, in the sense that the transition functions only close to a cocycle on triple overlaps. Now, ordinarily heterotic strings cannot couple to such twisted bundles, only D-branes can 
couple to such twisted bundles, as described in Section 2.3. Despite that fact, it was claimed in [90] [Section 3.1] that the $(0,4)$ theory nevertheless consistently couples to a twisted gauge bundle. In order to make that possible, the nonlinear sigma model was restricted to maps such that the pullback of the twisted bundle, is an honest bundle.

Such nonlinear sigma models, with a restriction on nonperturbative sectors, are equivalent to sigma models on gerbes, as reviewed in e.g. Section 2 , and so this is an example of a heterotic string compactification on a gerbe with a non-pullback bundle.

More generally, more of the analysis of [90] can also be rephrased in this language, following a discussion in [17][Section 12.3], which discussed how gerbes could be used to slightly simplify the analysis of the physical realization of geometric Langlands. Briefly, Hitchin moduli spaces arising from $G$ gauge theories are defined by modding out adjoint actions, under which the center $Z(G)$ is trivial and so formally one can replace them with moduli stacks which are $Z(G)$-gerbes. After reduction to two dimensions, one obtains sigma models on gerbes, which physics sees [17] as a sigma model on a disjoint union of spaces, matching results of [91].

In any event, after performing the dimensional reduction from a fourdimensional $\mathcal{N}=2$ theory to two dimensions, one gets [90] [Section 3.1] a heterotic sigma model on the Hitchin moduli space $\mathcal{M}_{H}(G, C)$, with a twisted bundle over that moduli space, twisted by an element of $H^{2}(Z(G))$. Since the Hitchin moduli space is defined by modding out the adjoint action of $G$, the center is trivial, and so one could naturally replace the Hitchin moduli space with a moduli stack which is a $Z(G)$ gerbe, just as in [17] [Section 12.3]. A heterotic sigma model on such a stack would appear to be a sigma model on the moduli space but with a restriction on allowed maps, exactly as described in [90] [Section 3.1].

As these two-dimensional $(0,2)$ theories are constructed by dimensional reduction of a consistent four-dimensional theory, it is difficult to believe that they are not consistent.

Other naively-consistent examples can be constructed in $(0,2)$ GLSM's. For example, consider the two examples:

- The rank 9 bundle

$$
0 \longrightarrow \mathcal{E} \longrightarrow \bigoplus_{1}^{9} \mathcal{O}(1) \oplus \mathcal{O}(10) \longrightarrow \mathcal{O}(19) \longrightarrow 0
$$

over $\mathbb{P}_{[3,3,3,3,6]}^{4}[18]$, a $\mathbb{Z}_{3}$ gerbe over $\mathbb{P}_{[1,1,1,1,2]}^{4}[6]$, 
- The rank 9 bundle

$$
0 \longrightarrow \mathcal{E} \longrightarrow \bigoplus_{1}^{9} \mathcal{O}(1) \oplus \mathcal{O}(13) \longrightarrow \mathcal{O}(22) \longrightarrow 0
$$

over $\mathbb{P}_{[3,3,6,9]}^{3}[21]$, a $\mathbb{Z}_{3}$ gerbe over $\mathbb{P}_{[1,1,2,3]}^{3}[7]$.

It is straightforward to check, just at the level of combinatorics, that they satisfy the usual conditions for a GLSM to be anomaly-free. However, the usual danger with GLSM's is that we do not have perfect control over the RG flow - although we have described them in terms of data associated to twisted bundles, along the RG flow they might pick up 'phases' (as suggested earlier), for example.

In the next subsections, we shall show explicitly that examples of this form do not yield consistent supersymmetric heterotic string compactifications, unfortunately.

\subsection{Cautionary example}

Let $\mathcal{E}$ be a rank 4 bundle on a Calabi-Yau $X$, defining a consistent $(0,2)$ SCFT. Now, consider a $\mathbb{Z}_{2}$ orbifold in which the orbifold group acts trivially on $X$, but by a sign flip on $\mathcal{E}$ (so that all of $\mathcal{E}$ is an eigenbundle of weight $-1)$.

This example can be shown to satisfy level-matching in the sense of [59], as well as the conditions in Appendix A.4. However, in principle this theory is nevertheless deeply suspicious. Since the $\mathbb{Z}_{2}$ acts trivially on right-moving fields, and only on left-moving fields, we could just as well think of this as a compactification of a ten-dimensional theory in which the left-moving GSO projection has been altered. Since the resulting new GSO does not coincide with either the existing $\operatorname{Spin}(32) / \mathbb{Z}_{2}$ or $E_{8} \times E_{8}$ strings, this theory must be inconsistent. (Indeed, this is the starting point for one construction of the ten-dimensional nonsupersymmetric $S O(8) \times S O(24)$ string $[76-78]$, though this orbifold must be supplemented by further phases.)

Another argument for inconsistency arises from considering massless spectra. Specifically, if we take $X$ to be a K3 surface, and consider a compactification of a ten-dimensional $E_{8} \times E_{8}$ string, in which the gauge bundle is embedded in one $E_{8}$, then the six-dimensional spectrum is anomalous. We summarize the details below, following the methods outlined in Appendix A. (The integer $n$ is the dimension of $X$; we will quickly specialize to $n=2$, but will remain general for as long as possible.) 
Following the appendix, there are two components in the inertia stack, which are identical:

$$
I_{\mathfrak{X}}=\mathfrak{X} \amalg \mathfrak{X} .
$$

Below we list results for both states and left and right $U(1)_{R}$ charges.

First, consider the untwisted sector.

In the $(\mathrm{R}, \mathrm{R})$ sector, the vacuum energy $E_{\mathrm{Id}}=0$. The massless charged states are

- $H^{m}\left(X, \wedge^{\text {even }} \mathcal{E}\right)$, charge $($ even $-2, m-n / 2)$, giving spacetime states valued in a spinor of $s o(8)$.

In the (NS, R) sector, the vacuum energy $E_{\mathrm{Id}}=-1$. The massless charged states are

- $H^{m}\left(X, \mathcal{E}^{*} \otimes \mathcal{E}\right)$, charge $(0, m-n / 2)$, spacetime gauge neutral,

- $H^{m}\left(X, \wedge^{2} \mathcal{E}\right)$, charge $(2, m-n / 2)$, spacetime gauge neutral,

- $H^{m}(X, \mathcal{O})$, charge $(0, m-n / 2)$, in the adjoint representation of $s o(8)$,

- $H^{m}\left(X, \wedge^{2} \mathcal{E}^{*}\right)$, charge $(-2, m-n / 2)$, spacetime gauge neutral.

Now, consider the twisted sector. Here, all of $\mathcal{E}$ is an eigenbundle with eigenvalue -1 .

In the $(\mathrm{R}, \mathrm{R})$ sector, $E=-1 / 2$. There are no massless charged states in this sector.

In the (NS, R) sector, $E=-1 / 2$. Again, there are no massless charged states in this sector.

States above are listed with charges $\left(q_{-}, q_{+}\right)$. The $q_{+}$charge distinguishes chiral multiplets from vector multiplets; the $q_{-}$charge is the charge of the $u(1)$ that combines with $s o(8)$ to build $s o(10)$.

For a compactification to four dimensions, $(n=3$,$) states with q_{+}=$ $-1 / 2$ would be spacetime fermions in chiral multiplets (and $q_{+}=+1 / 2$ their antichiral partners); states with $q_{+}=+3 / 2$ would be spacetime fermions in vector multiplets (and $q_{+}=-3 / 2$ their partners).

For a compactification to six dimensions, $(n=2$,) which is the pertinent case, states with $q_{+}= \pm 1$ are spacetime fermions in vector multiplets; states with $q_{+}=0$ are spacetime fermions in hypermultiplets.

Since we have a rank 4 bundle, in principle the $E_{8}$ should be broken to $\operatorname{Spin}(10)$, which in the worldsheet theory will be assembled from representations of $s o(8) \times u(1)$ (the $s o(8)$ rotating the remaining free left-moving fermions in the first $E_{8}$, and the $u(1)$ being an overall phase rotation on the bundle fermions, which on the $(2,2)$ locus would become the left $\mathrm{R}$ 
symmetry). Under the $s o(8) \times u(1)$ subalgebra, representations of $s o(10)$ decompose as follows:

$$
\begin{aligned}
\mathbf{4 5} & =\mathbf{8}_{-2} \oplus \mathbf{2 8}_{0} \oplus \mathbf{1}_{0} \oplus \mathbf{8}_{2}, \\
\mathbf{1 6} & =\mathbf{8}_{-1} \oplus \mathbf{8}_{+1}, \\
\mathbf{1 0} & =\mathbf{1}_{-2} \oplus \mathbf{8}_{0} \oplus \mathbf{1}_{2}, \\
\mathbf{1} & =\mathbf{1}_{0},
\end{aligned}
$$

where the subscript indicates the $q_{-}$charge.

We arrange the (untwisted sector) states into so(10) representations, with the following results:

- The adjoint of $s o(10)$ arises from $H^{*}(X, \mathcal{O})$. Contributing terms are:

- $H^{*}(X, \mathcal{O})$ in $(\mathrm{R}, \mathrm{R})$, transforming as $\mathbf{8}_{-2}$,

- $H^{*}\left(X, \wedge^{4} \mathcal{E} \cong \mathcal{O}\right)$ in $(\mathrm{R}, \mathrm{R})$, transforming as $\mathbf{8}_{+2}$,

- $H^{*}\left(X, \operatorname{Tr} \mathcal{E}^{*} \otimes \mathcal{E} \cong \mathcal{O}\right)$ in (NS, R), transforming as $\mathbf{1}_{0}$,

- $H^{*}(X, \mathcal{O})$ in (NS, R), transforming as $\mathbf{2 8}$.

- Copies of $\mathbf{1 0}$ of so(10) arise from $H^{*}\left(X, \wedge^{2} \mathcal{E}\right)$. Contributing terms are:

- $H^{*}\left(X, \wedge^{2} \mathcal{E}\right)$ in $(\mathrm{R}, \mathrm{R})$, transforming as $\mathbf{8}_{0}$,

- $H^{*}\left(X, \wedge^{2} \mathcal{E}\right)$ in (NS, R), transforming as $\mathbf{1}_{2}$,

- $H^{*}\left(X, \wedge^{2} \mathcal{E}^{*} \cong \wedge^{2} \mathcal{E}\right)$ in (NS, R), transforming as $\mathbf{1}_{-2}$.

- Gauge singlets, arising as $H^{*}(X$, End $\mathcal{E}$ ) (where we use End to denote the traceless endomorphisms), arising in the (NS, R) sector.

In addition, there is one vector in the adjoint representation of the second $E_{8}$, which is always present in computations of the form of Appendix A.

In any event, altogether in this six-dimensional theory we have

- $h^{0}(X, \mathcal{O})=1$ vector multiplets in the adjoint of $\operatorname{Spin}(10)$,

- One vector multiplet in the adjoint of $E_{8}$,

- $h^{1}\left(X, \wedge^{2} \mathcal{E}\right)=36$ half-hypermultiplet: ${ }^{16}$ in the $\mathbf{1 0}$ of $\operatorname{Spin}(10)$,

- 20 singlet hypermultiplets for $K 3$ moduli,

- $h^{1}($ End $\mathcal{E})=162$ singlet half-hypermultiplets for bundle moduli ${ }^{17}$.

16 The dimension of this sheaf cohomology group can be determined from index theory, and applies to any stable irreducible rank 4 bundle $\mathcal{E}$ on a K3 surface.

${ }^{17} \mathrm{It}$ is a standard result that the moduli in an irreducible rank $r$ vector bundle $\mathcal{E}$ on $\mathrm{K} 3$ with $c_{1}(\mathcal{E})=0, c_{2}(\mathcal{E})=c_{2}(T K 3)$ is encoded in $24 r+1-r^{2}$ hypermultiplets, or $2\left(24 r+1-r^{2}\right.$ half-hypermultiplets. Here, $r=4$. 
so that we find

$$
\begin{aligned}
n_{V} & =45+248=293, \\
n_{H} & =(1 / 2)((36)(10)+162)+20=281, \\
n_{H}-n_{V} & =-12 \neq 244,
\end{aligned}
$$

and so we see that this cannot satisfy anomaly cancellation, mechanically verifying our previous observation that this theory cannot be consistent.

More generally, any heterotic compactification on a gerbe, in which the bundle is twisted, will be of this same general type, unless the bundle has rank 8 and the trivially-acting group is $\mathbb{Z}_{2}$. Locally each theory will look like a compactification of a ten-dimensional theory with an altered GSO projection, and except for the case that the GSO projection switches between $\operatorname{Spin}(32) / \mathbb{Z}_{2}$ and $E_{8} \times E_{8}$, the resulting theory cannot be consistent.

For purposes of comparison, and to help illuminate the methods encoded in Appendix A, let us also outline the results in a closely related consistent compactification. If we did not orbifold, if we took a compactification of an $E_{8} \times E_{8}$ heterotic string on a smooth large-radius $K 3$ with a rank 4 vector bundle, then from a similar computation we would find

- $h^{0}(X, \mathcal{O})=1$ vector multiplets in the adjoint of $\operatorname{Spin}(10)$,

- One vector multiplet in the adjoint of $E_{8}$,

- $h^{1}(X, \mathcal{E})=16$ half-hypermultiplets in the $\mathbf{1 6}$ of $\operatorname{Spin}(10)$,

- $h^{1}\left(X, \Lambda^{2} \mathcal{E}\right)=36$ half-hypermultiplets in the $\mathbf{1 0}$ of $\operatorname{Spin}(10)$,

- $h^{1}\left(X, \Lambda^{3} \mathcal{E}=\mathcal{E}^{*}\right)=16$ half-hypermultiplets in the $\mathbf{1 6}$ of $\operatorname{Spin}(10)$,

- 20 singlet hypermultiplets for $K 3$ moduli,

- $h^{1}($ End $\mathcal{E})=162$ singlet half-hypermultiplets for bundle moduli,

where the representations of $\operatorname{Spin}(10)$ are constructed in the same fashion. Altogether, we find that

$$
\begin{aligned}
n_{V} & =45+248=293, \\
n_{H} & =(1 / 2)((16)(16)+(36)(10)+(16)(16)+162)+20=537, \\
n_{H}-n_{V} & =244,
\end{aligned}
$$

consistent with anomaly cancellation, in that standard compactification. Unfortunately, our gerbe example is not so well-behaved. 


\subsection{Second cautionary example}

For completeness, we give here a second cautionary example, here involving a heterotic $\operatorname{Spin}(32) / \mathbb{Z}_{2}$ compactification on a nontrivial toroidal orbifold. This will involve a rank 10 bundle over a $\mathbb{Z}_{2}$ gerbe on $\left[T^{4} / \mathbb{Z}_{2}\right]$, and although level matching holds, the spectrum is anomalous in six dimensions.

The $\mathbb{Z}_{2}$ gerbe is defined by $\left[T^{4} / \mathbb{Z}_{4}\right]$, where the $\mathbb{Z}_{4}$ acts on the $T^{4}$ by

$$
x \mapsto \exp \left(\frac{2 \pi i(2 k) k}{4}\right) x=(-)^{k} x
$$

so that there is a trivially-acting $\mathbb{Z}_{2}$ subgroup. (This is the same $\mathbb{Z}_{2}$ gerbe discussed in a different context in Section 4.2.) The gauge bundle is a rank 10 bundle, where the generator of $\mathbb{Z}_{4}$ acts on an $\mathcal{O}^{\oplus 2}$ factor by multiplication by $\exp (2 \pi i(2 / 4))=-1$, and on the $\mathcal{O}^{\oplus 8}$ factor by $\exp (2 \pi i / 4)$. It is straightforward to check that this satisfies level-matching, in the sense of [59], as well as the conditions in Appendix A.4.

Let us now outline the massless spectrum.

In the untwisted sector, there are massless states in the (NS, NS) sector. It is straightforward to compute $E_{\text {left }}=-1, E_{\text {right }}=-1 / 2$, and one has $\mathbb{Z}_{4^{-}}$ invariant states of the form

$$
\begin{array}{cc}
\text { State } & \text { Count } \\
\left(\lambda_{-1 / 2}^{1-6}, \bar{\lambda}_{-1 / 2}^{1-6}\right)^{2} \otimes\left(\psi_{-1 / 2}^{1-2}, \bar{\psi}_{-1 / 2}^{1-2}\right) & \begin{array}{c}
\text { spacetime vector, } \\
\bar{\partial} X_{-1}^{1-2} \otimes\left(\psi_{-1 / 2}^{1-2}, \bar{\psi}_{-1 / 2}^{1-2}\right)
\end{array} \\
\left(\lambda_{-1 / 2}^{7-14} \bar{\lambda}_{-1 / 2}^{7-14}\right) \otimes\left(\psi_{-1 / 2}^{1-2}, \bar{\psi}_{-1 / 2}^{1-2}\right) & \begin{array}{c}
\text { valued in adjoint of } s o(12) \\
\text { gravity, tensor multiplet contributions }
\end{array} \\
\left(\lambda_{-1 / 2}^{15-16}, \bar{\lambda}_{-1 / 2}^{15-16}\right)^{2} \otimes\left(\psi_{-1 / 2}^{1-2}, \bar{\psi}_{-1 / 2}^{1-2}\right) & \text { spacetime vector, } \\
\left(\lambda_{-1 / 2}^{1-6}, \bar{\lambda}_{-1 / 2}^{1-6}\right)\left(\lambda_{-1 / 2}^{15-16}, \bar{\lambda}_{-1 / 2}^{15-16}\right) \otimes\left(\psi_{-1 / 2}^{3-4}, \bar{\psi}_{-1 / 2}^{3-4}\right) & \text { valued in adjoint, } \mathbf{1} \text { (trace) of } s u(8) \\
\bar{\partial} X_{-1}^{3-4} \otimes\left(\psi_{-1 / 2}^{3-4}, \bar{\psi}_{-1 / 2}^{3-4}\right) & \text { spacetime vector, } \\
\text { valued in adjoint of } s o(4) & 4 \text { sets of scalars, } \\
\left(\left(\lambda_{-1 / 2}^{7-14},{ }^{2},\left(\bar{\lambda}_{-1 / 2}^{7-14}\right)^{2}\right) \otimes\left(\psi_{-1 / 2}^{3-4}, \bar{\psi}_{-1 / 2}^{3-4}\right)\right. & \text { valued in }(\mathbf{1 2}, \mathbf{4}) \text { of } s o(12) \times s o(4) \\
16 \text { scalars (toroidal moduli) }
\end{array}
$$

There are no massless states in the untwisted (R, NS) sector, and in fact also no massless states in the $k=1$ or $k=3$ sectors. 
All of the remaining massless states are in the $k=2$ sector. In the (NS, NS) sector, fields have the following boundary conditions:

$$
\begin{aligned}
X^{1-2}(\sigma+2 \pi) & =+X^{1-2}(\sigma), \\
X^{3-4}(\sigma+2 \pi) & =+X^{3-4}(\sigma), \\
\psi^{1-2}(\sigma+2 \pi) & =-\psi^{1-2}(\sigma), \\
\psi^{3-4}(\sigma+2 \pi) & =-\psi^{3-4}(\sigma), \\
\lambda^{1-6}(\sigma+2 \pi) & =-\lambda^{1-6}(\sigma) \\
\lambda^{7-14}(\sigma+2 \pi) & =-\exp \left(2 \pi i \frac{2}{4}\right) \lambda^{7-14}(\sigma)=+\lambda^{7-14}(\sigma), \\
\lambda^{15-16}(\sigma+2 \pi) & =-\lambda^{15-16}(\sigma) .
\end{aligned}
$$

It is straightforward to compute that $E_{\text {left }}=0, E_{\text {right }}=-1 / 2$. There is a multiplicity of left vacua, arising from $\lambda^{7-14}$. Let $|m\rangle$ denote a vacuum with $m+$ 's and $8-m$-'s, i.e. annihilated by $m \lambda^{\prime}$ 's and $8-m \bar{\lambda}$ 's, then under the action of the generator of $\mathbb{Z}_{4}$, it is straightforward to check that $|m=0,4,8\rangle$ are invariant, $|m=2,6\rangle$ get a sign flip, and the others are multiplied by various fourth roots of unity.

The $\mathbb{Z}_{4}$-invariant states in this sector are of the form

\begin{tabular}{cc} 
State & Count \\
\hline$|m=0,4,8\rangle \otimes\left(\psi_{-1 / 2}^{1-2}, \bar{\psi}_{-1 / 2}^{1-2}\right)$ & spacetime vector, valued in $\mathbf{1}, \mathbf{1}, \wedge^{4} \mathbf{8}=\mathbf{7 0}$ of $s u(8)$ \\
$|m=6,2\rangle \otimes\left(\psi_{-1 / 2}^{3-4}, \bar{\psi}_{-1 / 2}^{3-4}\right)$ & 4 sets of scalars, in $\wedge^{2} \mathbf{8}=\mathbf{2 8}, \wedge^{2} \overline{\mathbf{8}}=\overline{\mathbf{2 8}}$ of $s u(8)$
\end{tabular}
tions:

In the $k=2(\mathrm{R}, \mathrm{NS})$ sector, fields have the following boundary condi-

$$
\begin{aligned}
X^{1-2}(\sigma+2 \pi) & =+X^{1-2}(\sigma), \\
X^{3-4}(\sigma+2 \pi) & =+X^{3-4}(\sigma), \\
\psi^{1-2}(\sigma+2 \pi) & =-\psi^{1-2}(\sigma), \\
\psi^{3-4}(\sigma+2 \pi) & =-\psi^{3-4}(\sigma), \\
\lambda^{1-6}(\sigma+2 \pi) & =+\lambda^{1-6}(\sigma), \\
\lambda^{7-14}(\sigma+2 \pi) & =+\exp \left(2 \pi i \frac{2}{4}\right) \lambda^{7-14}(\sigma)=-\lambda^{7-14}(\sigma), \\
\lambda^{15-16}(\sigma+2 \pi) & =+\lambda^{15-16}(\sigma) .
\end{aligned}
$$

It is straightforward to compute that $E_{\text {left }}=0, E_{\text {right }}=-1 / 2$. There is a multiplicity of left vacua, as $\lambda^{1-6}$ and $\lambda^{15-16}$ are periodic. In particular, 
$| \pm \mp\rangle_{15-16}$ are invariant under $\mathbb{Z}_{4}$, whereas $| \pm \pm\rangle_{15-16}$ get a sign flip. Therefore, the $\mathbb{Z}_{4}$-invariant massless states are of the form

$$
\begin{array}{cc}
\text { State } & \text { Count } \\
\hline| \pm \cdots \pm\rangle_{1-6}| \pm \mp\rangle_{15-16} \otimes\left(\psi_{-1 / 2}^{1-2}, \bar{\psi}_{-1 / 2}^{1-2}\right) & \text { spacetime vector } \\
| \pm \cdots \pm\rangle_{1-6}| \pm \pm\rangle_{15-16} \otimes\left(\psi_{-1 / 2}^{3-4}, \bar{\psi}_{-1 / 2}^{3-4}\right) & \text { in }(\mathbf{3 2}, \mathbf{2}) \text { of } s o(12) \times s o(4) \\
& \text { in sets of scalars } \\
\left(\mathbf{3 2}^{\prime}, \mathbf{2}^{\prime}\right) \text { of } s o(12) \times s o(4)
\end{array}
$$

We can rearrange the spacetime vectors more sensibly as follows. The $s o(12) \times s o(4) \cong s o(12) \times s u(2) \times s u(2)$ is enhanced to an $e_{7} \times s u(2)$, using the fact that the adjoint representation of $e_{7}$ decomposes under $s o(12) \times$ $s u(2)$ as [73][Table 52]

$$
133=(66,1) \oplus(32,2) \oplus(\mathbf{1}, 3) .
$$

The 66 is the adjoint representation of $s o(12)$, which arises in $k=0$, as does the $\mathbf{3}$ of $s u(2)$ (half of the adjoint representation of so(4)), and the $(\mathbf{3 2}, \mathbf{2})$ arises in the sector $k=2$. Similarly, the $s u(8)$ is enhanced to $e_{7}$. The adjoint representation of $e_{7}$ decomposes under $s u(8)$ as [73] [Table 52]

$$
133=63 \oplus 70 .
$$

The $\mathbf{6 3}$ arises in the $k=0$ sector, and the $\mathbf{7 0}$ in $k=2$. In addition, there are three remaining vector multiplets, in the $k=0$ and $k=2$ sectors. Therefore, the complete gauge (algebra) symmetry in this compactification is $e_{7} \times e_{7} \times$ $s u(2) \times u(1)^{3}$.

The matter fields align themselves with the gauge algebra above. In the $k=0$ and $k=2$ sectors, the hypermultiplets valued in $\mathbf{2 8 ,} \overline{\mathbf{2 8}}$ of $s u(8)$ form hypermultiplets in the $\mathbf{5 6}$ of $e_{7}$, using the fact that under the $s u(8)$ subalgebra [73] [Table 52],

$$
\mathbf{5 6}=\mathbf{2 8} \oplus \overline{\mathbf{2 8}} .
$$

Similarly, since under the $s o(12) \times s u(2)$ subalgebra [73] [Table 52],

$$
56=\left(32^{\prime}, 1\right) \oplus(12,2),
$$

the $k=0$ hypermultiplet valued in $(\mathbf{1 2}, \mathbf{4})$ of $s o(12) \times s o(4)$ and the $k=2$ hypermultiplet valued in $\left(\mathbf{3 2}^{\prime}, \mathbf{2}^{\prime}\right)$ form a hypermultiplet valued in $(\mathbf{5 6}, \mathbf{2})$ of $e_{7} \times s u(2)$.

Let us summarize our results so far. We have found the following states: 
- 1 gravity multiplet,

- 1 tensor multiplet,

- 1 vector multiplet in the adjoint representation of $e_{7} \times e_{7} \times s u(2) \times$ $u(1)^{3}$,

- 2 hypermultiplets in the $(\mathbf{5 6}, \mathbf{1}, \mathbf{1})$ of $e_{7} \times e_{7} \times s u(2)$,

- 1 hypermultiplet in the $(\mathbf{1}, \mathbf{5 6}, \mathbf{2})$ of $e_{7} \times e_{7} \times s u(2)$,

- 4 singlet hypermultiplets.

It is straightforward to compute that there are 272 vector multiplets and 228 hypermultiplets. Since the difference is not 244 , this six-dimensional theory is anomalous.

\subsection{Third cautionary example}

Now consider an $E_{8} \times E_{8}$ string on a $\mathbb{Z}_{3}$ gerbe over a different $\left[T^{4} / \mathbb{Z}_{2}\right]$, constructed as $\left[T^{4} / \mathbb{Z}_{6}\right]$. Let the generator $g$ of $\mathbb{Z}_{6}$ act on the $T^{4}$ with coordinates $\left(X^{3}, X^{4}\right)$ as

$$
g:\left(X^{3}, X^{4}\right) \mapsto(\exp (+4 \pi i / 3), \exp (-4 \pi i / 3))
$$

Define a rank 2 bundle over this stack by taking $\mathcal{O}^{\oplus 2}$ over $T^{4}$, and let $g$ act with eigenvalues

$$
(\exp (-2 \pi i / 3), \exp (-4 \pi i / 3))
$$

It is straightforward to check that this satisfies anomaly cancellation in the sense of [59], and also the constraints in Appendix A.4.

In an $E_{8} \times E_{8}$ compactification, we can describe this as the following action on fields:

$$
\begin{aligned}
g \cdot X^{1-2} & =+X^{1-2}, \\
g \cdot X^{3} & =\exp (+4 \pi i / 3) X^{3}, \\
g \cdot X^{4} & =\exp (-4 \pi i / 3) X^{4}, \\
g \cdot \psi^{1-2} & =+\psi^{1-2} \\
g \cdot \psi^{3} & =\exp (+4 \pi i / 3) \psi^{3},
\end{aligned}
$$




$$
\begin{aligned}
g \cdot \psi^{4} & =\exp (-4 \pi i / 3) \psi^{4}, \\
g \cdot \lambda^{1-6} & =+\lambda^{1-6} \\
g \cdot \lambda^{7} & =\exp (-2 \pi i / 3) \lambda^{7}, \\
g \cdot \lambda^{8} & =\exp (-4 \pi i / 3) \lambda^{8} .
\end{aligned}
$$

Let us now outline the massless spectrum.

\begin{tabular}{|c|c|}
\hline State & Count \\
\hline $\bar{\partial} X_{-1}^{1-2} \otimes\left(\psi_{-1 / 2}^{1-2}, \bar{\psi}_{-1 / 2}^{1-2}\right)$ & gravity, tensor multiplet contributions \\
\hline$\left(\lambda_{-1 / 2}^{1-6}, \bar{\lambda}_{-1 / 2}^{1-6}\right)^{2} \otimes\left(\psi_{-1 / 2}^{1-2}, \bar{\psi}_{-1 / 2}^{1-2}\right)$ & vector in adjoint of $s o(12)$ \\
\hline$\left(\lambda_{-1 / 2}^{7} \lambda_{-1 / 2}^{8}, \bar{\lambda}_{-1 / 2}^{7} \bar{\lambda}_{-1 / 2}^{8}\right) \otimes\left(\psi_{-1 / 2}^{1-2}, \bar{\psi}_{-1 / 2}^{1-2}\right)$ & vectors in adjoint of $U(1)^{2}$ \\
\hline$\left(\lambda_{-1 / 2}^{7} \bar{\lambda}_{-1 / 2}^{7}, \lambda_{-1 / 2}^{8} \bar{\lambda}_{-1 / 2}^{8}\right) \otimes\left(\psi_{-1 / 2}^{1-2}, \bar{\psi}_{-1 / 2}^{1-2}\right)$ & vectors in adjoint of $U(1)^{2}$ \\
\hline$\lambda_{-1 / 2}^{8}\left(\lambda_{-1 / 2}^{1-6}, \bar{\lambda}_{-1 / 2}^{1-6}\right) \otimes\left(\psi_{-1 / 2}^{3}, \bar{\psi}_{-1 / 2}^{4}\right)$ & half-hypermultiplet in $\mathbf{1 2}$ of $s o(12)$ \\
\hline $\bar{\lambda}_{-1 / 2}^{8}\left(\lambda_{-1 / 2}^{1-6}, \bar{\lambda}_{-1 / 2}^{1-6}\right) \otimes\left(\bar{\psi}_{-1 / 2}^{3}, \psi_{-1 / 2}^{4}\right)$ & half-hypermultiplet in $\mathbf{1 2}$ of so(12) \\
\hline$\lambda_{-1 / 2}^{7}\left(\lambda_{-1 / 2}^{1-6}, \bar{\lambda}_{-1 / 2}^{1-6}\right) \otimes\left(\bar{\psi}_{-1 / 2}^{3}, \psi_{-1 / 2}^{4}\right)$ & half-hypermultiplet in $\mathbf{1 2}$ of so(12) \\
\hline $\bar{\lambda}_{-1 / 2}^{7}\left(\lambda_{-1 / 2}^{1-6}, \bar{\lambda}_{-1 / 2}^{1-6}\right) \otimes\left(\psi_{-1 / 2}^{3}, \bar{\psi}_{-1 / 2}^{4}\right)$ & half-hypermultiplet in $\mathbf{1 2}$ of $s o(12)$ \\
\hline$\left(\bar{\partial} X^{3}, \bar{\partial} X^{4}\right) \otimes\left(\bar{\psi}_{-1 / 2}^{3}, \psi_{-1 / 2}^{4}\right)$ & 1 singlet hypermultiplet \\
\hline$\left(\overline{\partial X}^{3}, \bar{\partial} X^{4}\right) \otimes\left(\psi_{-1 / 2}^{3}, \bar{\psi}_{-1 / 2}^{4}\right)$ & 1 singlet hypermultiplet \\
\hline$\lambda_{-1 / 2}^{7} \bar{\lambda}_{-1 / 2}^{8} \otimes\left(\psi_{-1 / 2}^{3}, \bar{\psi}_{-1 / 2}^{4}\right)^{\prime}$ & $1 / 2$ singlet hypermultiplet \\
\hline $\bar{\lambda}_{-1 / 2}^{7} \lambda_{-1 / 2}^{8} \otimes\left(\bar{\psi}_{-1 / 2}^{3}, \psi_{-1 / 2}^{4}\right)$ & $1 / 2$ singlet hypermultiplet \\
\hline
\end{tabular}

In the untwisted sector, there are massless states in the (NS, NS) sector. It is straightforward to compute that $E_{\text {left }}=-1, E_{\text {right }}=-1 / 2$, and one has $\mathbb{Z}_{6}$-invariant states of the form

There are no massless states in the untwisted (R, NS) sector, and no massless states in $k=1, k=2$ sectors. The $k=3,4,5$ sectors are copies of the $k=0,1,2$ sectors, respectively. Thus, altogether, the spectrum is two copies of the states above.

Note that since there are no $(\mathrm{R}, \mathrm{NS})$ states, the nonabelian gauge symmetry is only $s o(12)$; it is not enhanced to $e_{7}$. Also, since the spectrum is two copies of the states above, the spectrum contains two gravitons, and hence would be a likely candidate for decomposition.

Unfortunately, the spectrum is also anomalous. The gauge symmetry is $s o(12) \times e_{8} \times u(1)^{4}$ (including the second $E_{8}$, which until now has been suppressed), so the total number of vector multiplets is 318 , and the number 
of hypermultiplets is 27 . Clearly $n_{H}-n_{V} \neq 244$, so this model is anomalous in six dimensions.

\subsection{Potential refinements of anomaly cancellation}

So far we have described some consistent $(0,2)$ SCFT's of the class III form, and also illustrated in detail how theories of this form cannot be consistently used in supersymmetric heterotic string compactifications. This begs the question of whether there exists a criterion, perhaps a generalization of anomaly cancellation, that can be used to distinguish theories of this form. In this section, we will examine one such possibility.

In Appendix C we describe a modified notion of Chern classes and characters, labelled $c^{\text {rep }}$ and $\mathrm{ch}^{\text {rep}}$, that contain extra information in twisted sectors. It is tempting to speculate that one might be able to use these to obtain additional finite-group anomaly constraints on theories by demanding matching $\operatorname{ch}_{2}^{\text {rep }}$ 's. Let us check this by studying GLSM's, for which anomaly cancellation conditions are more or less well understood. We will argue that

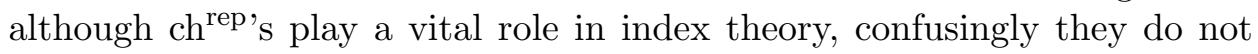
seem to define any new anomaly-cancellation conditions.

Consider a $(0,2)$ theory over the hypersurface $\mathfrak{X}=\mathbb{P}_{[k, k, \ldots, k]}^{n}[d]$, with gauge bundle $\mathcal{E}$ :

$$
0 \longrightarrow \mathcal{E} \longrightarrow \oplus_{a} \mathcal{O}\left(n_{a}\right) \longrightarrow \mathcal{O}(m) \longrightarrow 0
$$

It is straightforward to compute that

$$
\begin{aligned}
\left.c_{1}^{\mathrm{rep}}(T \mathfrak{X})\right|_{\alpha} & =(n+1) \frac{k}{k} J-\frac{d}{k} \alpha^{-d} J \\
\left.\operatorname{ch}_{2}^{\mathrm{rep}}(T \mathfrak{X})\right|_{\alpha} & =\left.\operatorname{ch}_{2}^{\mathrm{rep}}\left(\oplus_{n+1} \mathcal{O}(k)\right)\right|_{\alpha}-\left.\operatorname{ch}_{2}^{\mathrm{rep}}(\mathcal{O}(d))\right|_{\alpha}, \\
& =\frac{1}{2}(n+1)\left(\frac{k}{k} J\right)^{2}-\frac{1}{2}\left(\frac{d}{k} J\right)^{2} \alpha^{-d},
\end{aligned}
$$

and for the bundle $\mathcal{E}$,

$$
\begin{aligned}
\left.c_{1}^{\mathrm{rep}}(\mathcal{E})\right|_{\alpha} & =\sum_{a} \frac{n_{a}}{k} J \alpha^{-n_{a}}-\frac{m}{k} J \alpha^{-m} \\
\left.\operatorname{ch}_{2}^{\mathrm{rep}}(\mathcal{E})\right|_{\alpha} & =\left.\operatorname{ch}_{2}^{\mathrm{rep}}\left(\oplus_{a} \mathcal{O}\left(n_{a}\right)\right)\right|_{\alpha}-\left.\operatorname{ch}_{2}^{\mathrm{rep}}(\mathcal{O}(m))\right|_{\alpha}, \\
& =\frac{1}{2} \sum_{a}\left(\frac{n_{a}}{k} J\right)^{2} \alpha^{-n_{a}}-\frac{1}{2}\left(\frac{m}{k} J\right)^{2} \alpha^{-m}
\end{aligned}
$$


By contrast, anomaly cancellation in the GLSM is merely the statement that

$$
\sum_{a} n_{a}^{2}-m^{2}=(n+1) k^{2}-d^{2}
$$

a much weaker statement than demanding $\operatorname{ch}_{2}^{\text {rep }}(\mathcal{E})=\operatorname{ch}_{2}^{\text {rep }}(T \mathfrak{X})$ in each sector $\alpha$. Anomaly cancellation in the GLSM is well-understood - in the present case, this is just the gauge anomaly in a $U(1)$ gauge theory, which is

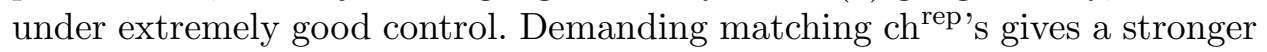
condition - some theories that would satisfy GLSM anomaly cancellation, would not satisfy the constraint of matching $\mathrm{ch}^{\text {rep's. }}$

For this reason, we do not believe that one should demand matching

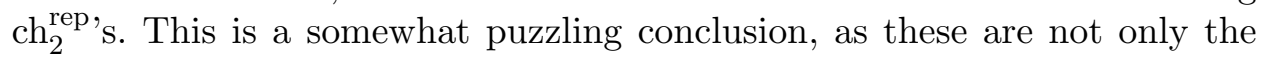
most natural notion of Chern classes on stacks, but they are also vital in index theory, which ordinarily would be a route to deriving their utility. (On the other hand, we briefly remark on a possible application of $c_{1}^{\text {rep }}$ in Appendix A.4.)

\section{Combinations}

So far we have discussed three fundamental classes of examples of heterotic string compactifications on gerbes.

Those three classes do not exhaust all possibilities; rather, one should think of them as 'building blocks' that can be used to assemble more complicated possibilities.

For one example, consider a string on a $\mathbb{Z}_{4}$ gerbe, of which a $\mathbb{Z}_{2}$ subgroup acts on a rank 8 bundle, but the $\mathbb{Z}_{2}$ coset leaves the bundle invariant. A version of the decomposition conjecture should apply here, relating this $(0,2)$ SCFT to a disjoint union of two $(0,2)$ SCFT's, each of which would involve a heterotic string on a $\mathbb{Z}_{2}$ gerbe with a nontrivial action on the gauge bundle. Those individual SCFT's might be dual to a different string compactification (class II), or might not define a consistent heterotic string compactification (class III).

It is straightforward to assemble more complicated possibilities, following similar patterns.

\section{Conclusions}

In this paper we have examined general aspects of heterotic string compactifications on generalized spaces known as stacks, focusing on the particularly interesting special case of stacks that are gerbes. 
Briefly, we have described how heterotic string compactifications on gerbes are built from three basic classes:

- In the special case that the gauge bundle on the gerbe is a pullback from an underlying space, the heterotic theory on the gerbe is equivalent to a heterotic theory on a disjoint union of spaces, the same sort of decomposition as type II strings on gerbes [17].

- In the special case that the gauge bundle on the gerbe is twisted in such a way as to locally duplicate a different ten-dimensional GSO projection, the gerbe compactification seems to be dual to a compactification of the corresponding different heterotic string.

- In other cases in which the gauge bundle is different from a pullback from the base, although at least sometimes one can define consistent $(0,2)$ SCFT's, there do not seem to be any viable perturbative heterotic string compactifications.

There are several open questions that would be interesting to pursue. For example,

- We have not identified a complete set of sufficient conditions for a stack $\mathfrak{X}$ with bundle $\mathcal{E} \rightarrow \mathfrak{X}$ to define a consistent heterotic string compactification. We have identified a number of necessary conditions, such as anomaly cancellation on the cover and level-matching in orbifolds, we have derived additional necessary conditions from well-definedness of Fock vacua, but we have also observed that these conditions do not suffice in general. We have speculated on some enhancements of anomaly cancellation (involving the $\mathrm{ch}^{\text {rep's that }}$ can be defined on stacks), but do not at this time have any definitive statements to make.

- We have discussed a heterotic analogue of the decomposition conjecture for banded gerbes, with bundle a pullback from the base. We do not at this time have an analogue for nonbanded gerbes.

These questions are left for future work.

\section{Acknowledgements}

Various parts of this work have been in progress for approximately five years, and so we have a number of people to thank, including M. Ando (for discussions of elliptic genera with twisted bundles), P. Clarke (for assistance constructing Distler-Kachru examples), K. Dienes (for discussions of 
free fermion models and sufficiency of level matching), J. Distler, D. Freed

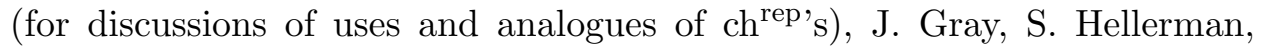
I. Melnikov, E. Scheidegger, K. Wendland (for discussions of the nonsupersymmetric $S O(8) \times S O(24)$ string), and especially T. Pantev for very many useful discussions and collaborations revolving around stacks.

L. Anderson was supported by the Fundamental Laws Initiative of the Center for the Fundamental Laws of Nature, Harvard University. B. Ovrut was supported in part by the DOE under contract DE-AC02-76-ER-03071 and the NSF under grant 1001296. Over the course of this work, E. Sharpe was partially supported by NSF grants DMS-0705381, PHY-0755614, and PHY-1068725.

\section{Appendix A. Massless spectra of heterotic strings on stacks}

\section{A.1. Basic definitions}

In this section, we will describe the computation of the massless spectrum of a perturbative heterotic $E_{8} \times E_{8}$ string compactified on a smooth DeligneMumford stack $\mathfrak{X}$ with suitable gauge bundle $\mathcal{E} \rightarrow \mathfrak{X}$, following (in spirit when not detail) 65] and [92. Not only will this be useful for computations, but the existence of such a computational method is a good consistency check for the existence of heterotic string compactifications on stacks.

Let $\mathfrak{X}$ be a smooth Deligne-Mumford stack of complex dimension ${ }^{18} n \leq$ 4 , and $\mathcal{E}$ a holomorphic vector bundle over $\mathfrak{X}$ of rank $r$, satisfying suitable anomaly-cancellation conditions. We will embed the bundle in one of the $E_{8}$ 's of the ten-dimensional heterotic string, so we will assume that $r<8$. As in 65, all our computations will be in a right-moving $\mathrm{R}$ sector (hence, spacetime fermions), but spacetime supersymmetry can be used to derive the NS sector (spacetime bosons) in principle.

Let $I_{\mathfrak{X}}$ denote the inertia stack associated to $\mathfrak{X}$. Roughly speaking, the inertia stack is a geometric mechanism for encoding twisted sectors; it has multiple components, each of which corresponds to a twisted sector in a standard global orbifold. For example, if $\mathfrak{X}=\left[\mathbb{C}^{2} / \mathbb{Z}_{2}\right]$, where the $\mathbb{Z}_{2}$ acts by sign flips, then

$$
I_{\mathfrak{X}}=\left[\mathbb{C}^{2} / \mathbb{Z}_{2}\right] \amalg\left[\text { point } / \mathbb{Z}_{2}\right] .
$$

\footnotetext{
${ }^{18}$ For simplicity, as we wish to work in light-cone gauge, we will assume that the complex dimension is bounded by 4 .
} 
For another example, if $\mathfrak{X}=\left[\mathbb{C} / \mathbb{Z}_{3}\right]$, where the $\mathbb{Z}_{3}$ acts by multiplying by phases, then

$$
I_{\mathfrak{X}}=\left[\mathbb{C} / \mathbb{Z}_{3}\right] \amalg\left[\operatorname{point} / \mathbb{Z}_{3}\right] \amalg\left[\text { point } / \mathbb{Z}_{3}\right] .
$$

For yet another example, if $\mathfrak{X}=\left[\mathbb{C} / \mathbb{Z}_{2}\right]$, where the $\mathbb{Z}_{2}$ acts trivially (so that all of $\mathbb{C}$ is fixed), then

$$
I_{\mathfrak{X}}=\left[\mathbb{C} / \mathbb{Z}_{2}\right] \amalg\left[\mathbb{C} / \mathbb{Z}_{2}\right] .
$$

(See e.g. [48 51, 53, 58] for more information on the inertia stack.) In general, points in the inertia stack are pairs $(x, \alpha)$, where $x$ is a point of $\mathfrak{X}$, and $\alpha$ is an automorphism of $x$, which for an orbifold $[Y / G]$ by $G$ a finite group, would define the twisted sectors. In the $\left[\mathbb{C}^{3} / \mathbb{Z}_{3}\right]$ example, if $g$ generates $\mathbb{Z}_{3}$, then the two copies of $\left[\right.$ point $\left./ \mathbb{Z}_{3}\right]$ correspond to $\alpha=g, g^{2}$. The inertia stack $I_{\mathfrak{X}}$ always contains a copy of $\mathfrak{X}$ as one component, corresponding to $\alpha=\mathrm{Id}$.

Let us describe how to compute the spectrum on each component $\alpha$ of $I_{\mathfrak{X}}$. (We will use $\alpha$ to denote both a component of $I_{\mathfrak{X}}$ and the automorphism defining that component.)

First, let $q: I_{\mathfrak{X}} \rightarrow \mathfrak{X}$ denote the natural projection onto a single component, and for $\alpha \neq \mathrm{Id}$, decompose the pullback bundles into eigenbundle: 19 of $\langle\alpha\rangle$ :

$$
\begin{aligned}
\left.q^{*} T \mathfrak{X}\right|_{\alpha} & =\oplus_{n} T_{n}^{\alpha}, \\
\left.q^{*} \mathcal{E}\right|_{\alpha} & =\oplus_{n} \mathcal{E}_{n}^{\alpha} .
\end{aligned}
$$

Define $t_{\alpha}$ to be the order of the corresponding automorphism, and take $T_{n}^{\alpha}$ and $\mathcal{E}_{n}^{\alpha}$ to be associated with character

$$
\exp \left(2 \pi i n / t_{\alpha}\right)
$$

By this we mean that the (R-sector) worldsheet fermions corresponding to $T_{n}^{\alpha}$ and $\mathcal{E}_{n}^{\alpha}$ have boundary conditions of the form

$$
\psi(\sigma+2 \pi)=\exp \left(2 \pi i n / t_{\alpha}\right) \psi(\sigma) .
$$

We will denote fermions couplings to $T_{n}^{\alpha}$ (respectively, $\mathcal{E}_{n}^{\alpha}$ ) by $\psi_{+, n}$ (respectively, $\left.\lambda_{-, n}\right)$.

19 Since $\alpha$ leaves the points invariant, this component of the inertia stack must have a $\langle\alpha\rangle$ gerbe structure, and bundles on such gerbes have an eigenbundle decomposition as given here. 
Let us pause to briefly discuss some concrete examples, to illuminate these abstract definitions. For global orbifolds by finite groups, it should hopefully be clear that the description above is an abstraction of the standard prescription for distinguishing various worldsheet fermions with different boundary conditions. Let us turn to an example which does not have such a realization, but which is relevant to $(0,2)$ GLSMs. Take $\mathfrak{X}=\mathbb{P}_{[1,1,1,2,2]}^{4}$, with bundle

$$
0 \longrightarrow \mathcal{E} \longrightarrow \oplus_{a} \mathcal{O}\left(n_{a}\right) \stackrel{F_{a}}{\longrightarrow} \mathcal{O}(m) \longrightarrow 0
$$

where $\operatorname{det} \mathcal{E}^{*} \cong K_{\mathfrak{X}}$ :

and second Chern classes match:

$$
\sum n_{a}-m=7
$$

$$
\sum n_{a}^{2}-m^{2}=11
$$

This is not Calabi-Yau, so it would not be directly useful for a string compactification, but can help illuminate some general aspects. This stack has a $\mathbb{P}^{1}$ of $\mathbb{Z}_{2}$ orbifolds, so the inertia stack has the form

$$
I_{\mathfrak{X}}=\mathfrak{X} \amalg \mathbb{P}_{[2,2]}^{1} .
$$

On the nontrivial component $\mathbb{P}_{[2,2]}^{1}$, call it $\alpha$, we can work out the decomposition of the gauge bundle. Suppose, for example, that $m$ is odd. For any given $a$, if $n_{a}$ is even, then $F_{a}$ is odd, so $F_{a}=0$; if $n_{a}$ is even on the other hand, there is no constraint on $F_{a}$. In this case, we can decompose

$$
\left.q^{*} \mathcal{E}\right|_{\alpha}=\mathcal{E}_{+} \oplus \mathcal{E}_{-}
$$

where $\mathcal{E}_{+}$is invariant, $\mathcal{E}_{-}$anti-invariant under $\mathbb{Z}_{2}$, and specifically

$$
\begin{aligned}
& \mathcal{E}_{+}=\oplus \mathcal{O}\left(n_{a} \text { even }\right) \\
& \mathcal{E}_{-}=\operatorname{ker}\left(\oplus \mathcal{O}\left(n_{a} \text { odd }\right) \longrightarrow \mathcal{O}(m)\right)
\end{aligned}
$$

A closely related decomposition exists for $m$ even.

Now that we have illuminated the definitions, let us return to our description of the general procedure for spectrum computation. At this point, the computation of spectra becomes more or less identical to that in an ordinary global orbifold by a finite group, if we think of $\alpha$ as denoting a twisted sector. We will walk through the details, as there are a few important subtleties for general cases not usually discussed in the literature, especially regarding Fock vacua, but the rest of the computation is nearly standard, once one masters the description. 


\section{A.2. Vacuum energies}

We need to compute left- and right-moving zero point energies in each twisted sector. Recall that a complex worldsheet fermion $\psi$ with boundary conditions

$$
\psi(\sigma+2 \pi)=\exp (i(\pi+\theta)) \psi(\sigma), \quad-\pi \leq \theta \leq \pi
$$

contributes

$$
-\frac{1}{24}+\frac{1}{8}\left(\frac{\theta}{\pi}\right)^{2}
$$

to the vacuum energy, and a complex boson with the same boundary conditions contributes with the opposite sign.

Let $\theta_{n}^{T, \alpha}$ denote the $\theta$ corresponding to worldsheet fermions associated with $T_{n}^{\alpha}$, and $\theta_{n}^{\mathcal{E}, \alpha}$ denote the $\theta$ corresponding to worldsheet fermions associated with $\mathcal{E}_{n}^{\alpha}$. For the moment, we will assume that we are in an $(\mathrm{R}, \mathrm{R})$ sector (meaning, left-moving fermions in the first $E_{8}$ and right-moving fermions in an R sector, second $E_{8}$ will be held fixed in an NS sector). In an (NS, R) sector (left-moving fermions in the first $E_{8}$ in an NS sector instead), we would modify the $\theta$ 's for left-moving worldsheet fermions to take into account an extra sign in boundary conditions.

Then, in an $(R, R)$ sector, the left-moving vacuum energy is

$$
\begin{aligned}
E_{(\mathrm{R}, \mathrm{R}) \mathrm{Id}} & =8\left(-\frac{1}{24}\right)+8\left(+\frac{1}{12}\right)+4\left(-\frac{1}{12}\right), \\
& =0,
\end{aligned}
$$

in the untwisted sector $(\alpha=\mathrm{Id})$ and in twisted sectors,

$$
\begin{aligned}
E_{(\mathrm{R}, \mathrm{R}), \alpha}= & 8\left(-\frac{1}{24}\right)+\sum_{n}\left(\operatorname{rk} \mathcal{E}_{n}^{\alpha}\right)\left(-\frac{1}{24}+\frac{1}{8}\left(\frac{\theta_{n}^{\mathcal{E}, \alpha}}{\pi}\right)^{2}\right)+(8-r)\left(+\frac{1}{12}\right) \\
& +\sum_{n}\left(\operatorname{rk} T_{n}^{\alpha}\right)\left(+\frac{1}{24}-\frac{1}{8}\left(\frac{\theta_{n}^{T, \alpha}}{\pi}\right)^{2}\right)+(4-n)\left(-\frac{1}{12}\right), \\
= & \frac{n-r}{8}+\frac{1}{8} \sum_{n}\left(\operatorname{rk} \mathcal{E}_{n}^{\alpha}\right)\left(\frac{\theta_{n}^{\mathcal{E}, \alpha}}{\pi}\right)^{2}-\frac{1}{8} \sum_{n}\left(\operatorname{rk} T_{n}^{\alpha}\right)\left(\frac{\theta_{n}^{T, \alpha}}{\pi}\right)^{2} .
\end{aligned}
$$

In all cases the right-moving vacuum energy vanishes, since the right-moving bosons and fermions make equal and opposite contributions. 
Vacuum energies in (NS, R) sectors (meaning, left-moving fermions of the first $E_{8}$ in an NS sector) can be computed similarly. For completeness, we list them below: in an untwisted sector,

$$
\begin{aligned}
E_{(\mathrm{NS}, \mathrm{R}), \mathrm{Id}} & =8\left(-\frac{1}{24}\right)+8\left(-\frac{1}{24}\right)+4\left(-\frac{1}{12}\right), \\
& =-1,
\end{aligned}
$$

and in a twisted sector,

$$
\begin{aligned}
E_{(\mathrm{NS}, \mathrm{R}), \alpha}= & 8\left(-\frac{1}{24}\right)+\sum_{n}\left(\operatorname{rk} \mathcal{E}_{n}^{\alpha}\right)\left(-\frac{1}{24}+\frac{1}{8}\left(\frac{\tilde{\theta}_{n}^{\mathcal{E}, \alpha}}{\pi}\right)^{2}\right)+(8-r)\left(-\frac{1}{24}\right) \\
& +\sum_{n}\left(\operatorname{rk} T_{n}^{\alpha}\right)\left(+\frac{1}{24}-\frac{1}{8}\left(\frac{\theta_{n}^{T, \alpha}}{\pi}\right)^{2}\right)+(4-n)\left(-\frac{1}{12}\right) \\
= & -1+\frac{n}{8}+\frac{1}{8} \sum_{n}\left(\operatorname{rk} \mathcal{E}_{n}^{\alpha}\right)\left(\frac{\tilde{\theta}_{n}^{\mathcal{E}, \alpha}}{\pi}\right)^{2}-\frac{1}{8} \sum_{n}\left(\operatorname{rk} T_{n}^{\alpha}\right)\left(\frac{\theta_{n}^{T, \alpha}}{\pi}\right)^{2},
\end{aligned}
$$

where $\tilde{\theta}$ denotes $\theta$ 's as modified to include a sign in the boundary conditions. Vacuum energies in (NS, R) sectors (meaning, left-moving fermions of the first $E_{8}$ in an NS sector) can be computed similarly.

\section{A.3. Fock vacua}

The fractional charges of the Fock vacua can and should be understood in terms of coupling to nontrivial bundles. Recall (see e.g. [74]) that a complex left-moving fermion $\lambda$ with boundary conditions

$$
\lambda(\sigma+2 \pi)=e^{-i \theta} \lambda(\sigma)
$$

contributes fractional fermion number

$$
\frac{\theta}{2 \pi}-\left[\frac{\theta}{2 \pi}\right]-\frac{1}{2}
$$

and a complex right-moving fermion $\psi$ with the same boundary conditions contributes fractional fermion number

$$
-\left(\frac{\theta}{2 \pi}-\left[\frac{\theta}{2 \pi}\right]-\frac{1}{2}\right)
$$


In the present case, in the sector defined by automorphism $\alpha$, we have complex left-moving fermions $\lambda_{-, n}$ coupling to bundle $\mathcal{E}_{n}^{\alpha}$, with boundary conditions

$$
\lambda_{-, n}(\sigma+2 \pi)=\exp \left(2 \pi i n / t_{\alpha}\right) \lambda_{-, n}(\sigma)
$$

and complex right-moving fermions $\psi_{+, n}$ coupling to bundle $T_{n}^{\alpha}$, with boundary conditions

$$
\psi_{+, n}(\sigma+2 \pi)=\exp \left(2 \pi i n / t_{\alpha}\right) \psi_{+, n}(\sigma)
$$

Putting this together, we see that from each set of $\lambda_{-, n}$, the Fock vacuum couples to

$$
\left(\operatorname{det} \mathcal{E}_{n}^{\alpha}\right)^{-\frac{n}{t_{\alpha}}-\left[-\frac{n}{t_{\alpha}}\right]-\frac{1}{2}}
$$

and from each set of $\psi_{+, n}$, the Fock vacuum couples to

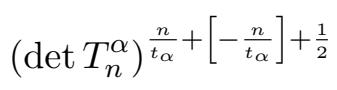

Since the $\alpha$-sector has components which are $t_{\alpha}$ gerbes, $t_{\alpha}$-th roots of bundles might exist, though not necessarily. (See Appendix D for examples of bundles on $\mathbb{Z}_{n}$-gerbes which do and do not admit $n$th roots.) Existence of these roots is a necessary condition for the existence of the physical theories. When multiple roots exist, as will happen if the components are not simplyconnected, the roots must be specified as part of the data defining the sigma model.

When there are periodic fermions, there are multiple Fock vacua, each with different (fractional) charges. The different Fock vacua are defined by which subset of the fermi zero modes annihilate. In our case, we will work in conventions in which our Fock vacuum $|0\rangle$ has the properties

$$
\lambda_{-, 0}^{a}|0\rangle=0=\psi_{+, 0}^{\bar{\imath}}|0\rangle .
$$

As before, reflecting the fact that the $\lambda$ 's and $\psi$ 's couple to nontrivial bundles, this Fock vacuum is itself a section of a line bundle. From those periodic fermions, the Fock vacuum behaves as a section of a square root of 
the determinant of the periodic modes, specifically,

$$
\sqrt{K_{\alpha} \otimes \operatorname{det} \mathcal{E}_{0}^{\alpha}}
$$

(square root chosen with periodic boundary conditions), where

$$
K_{\alpha}=\operatorname{det}\left(T_{0}^{\alpha}\right)^{*}
$$

i.e. the canonical bundle of the $\alpha$ component of $I_{\mathfrak{X}}$. (Note that in an (NS, R) sector, the 'invariant' subbundle $\mathcal{E}_{0}$ is defined to be invariant under the combination of spacetime group action and spin state boundary condition, and hence will be different from the $\mathcal{E}_{0}$ in an $(\mathrm{R}, \mathrm{R})$ sector.) If the square root above does not exist, then the orbifold is not well-defined, which we shall come back to after we derive the expression above.

We can derive the result above for periodic fermions as follows. Different choices of Fock vacua act as sections of different line bundles, related by fermions acting as raising and lowering operators. Just as in fractional charges, the square root and bundles above are constrained by the fact that the set of Fock vacua must be consistent with those raising and lowering operations. For example, the 'opposite' Fock vacuum $|0\rangle^{\text {op }}$ is defined by applying raising operators maximally:

$$
|0\rangle^{\mathrm{op}}=\lambda_{-, 0}^{\bar{a}_{1}} \cdots \lambda_{-, 0}^{\bar{a}_{r}} \psi_{+, 0}^{i_{1}} \cdots \psi_{+, 0}^{i_{d}}|0\rangle
$$

(where $r$ is the rank of $\mathcal{E}_{0}^{\alpha}$ and $d$ the rank of $T_{0}^{\alpha}$ ), so if our Fock vacuum $|0\rangle$ couples to a line bundle $\mathcal{L}$, then the opposite or dual Fock vacuum above must couple to

$$
\left(\operatorname{det} \mathcal{E}_{0}^{\alpha *}\right) \otimes\left(\operatorname{det} T_{0}^{\alpha}\right) \otimes \mathcal{L}
$$

which, by symmetry, should also be the same as $\mathcal{L}^{*}$. In other words,

$$
\left(\operatorname{det} \mathcal{E}_{0}^{\alpha *}\right) \otimes\left(\operatorname{det} T_{0}^{\alpha}\right) \otimes \mathcal{L} \cong \mathcal{L}^{*}
$$

or more simply

$$
\mathcal{L}^{2} \cong\left(\operatorname{det} \mathcal{E}_{0}^{\alpha}\right) \otimes\left(\operatorname{det} T_{0}^{\alpha *}\right)=K_{\alpha} \otimes \operatorname{det} \mathcal{E}_{0}^{\alpha}
$$

from which our claim is derived. In particular, taking $\mathcal{L}=\mathcal{O}$ will not, in general, be consistent. 
In passing, note that the set of all Fock vacua in sector $\alpha$ form a vector bundle

$$
\begin{aligned}
\left(\wedge^{\bullet} \mathcal{E}_{0}^{\alpha *}\right) \otimes\left(\wedge^{\bullet} T_{0}^{\alpha}\right) & \otimes \sqrt{K_{\alpha} \otimes \operatorname{det} \mathcal{E}_{0}^{\alpha}} \\
& \otimes \otimes_{n>0}\left(\left(\operatorname{det} \mathcal{E}_{n}^{\alpha}\right)\left(\operatorname{det} T_{n}^{\alpha}\right)^{-1}\right)^{-\frac{n}{t_{\alpha}}-\left[-\frac{n}{t_{\alpha}}\right]-\frac{1}{2}}
\end{aligned}
$$

over $\left.I_{\mathfrak{X}}\right|_{\alpha}$, taking into account contributions from all boundary conditions.

The phenomenon of Fock vacua coupling to nontrivial bundles has also been noted in this context in [92, 93] [Section 2.1]. However, aside from those two sources, we are not aware of many discussions of Fock vacua as sections of line bundles over target spaces ${ }^{20}$ in the literature, so it is perhaps useful to elaborate on this point. As we shall see in the present case and also in [92], it plays a crucial role in closing the spectrum under Serre duality of the sheaf cohomology groups, a basic symmetry of the spectra discussed in [65]. The same behavior also arises elsewhere. For example, in open string theories, the Fock vacuum also transforms as a section of a line bundle, a square root of the canonical bundle of the D-brane worldvolume $B$ (assumed Spin), if the D-brane worldvolume is not Calabi-Yau. This can be understood simply from the matter representations: a spinor in the worldvolume theory can be represented mathematically in the form [96]

$$
\left(\wedge^{\bullet} T B\right) \otimes \sqrt{K_{B}}
$$

In terms of the worldsheet RNS formalism, perturbative modes realize the $T B$ factors, and the $\sqrt{K_{B}}$ is implemented by the Fock vacuum itself. This phenomenon is also reminiscent of factors arising from the Freed-Witten anomaly [97, 98], though we shall not pursue that direction here.

\section{A.4. Consistency conditions derived from existence of Fock vacua}

In some cases, the $t_{\alpha}$ th roots A.1, A.2 or the square root A.3 might not ${ }^{21}$ exist as honest equivariant line bundles. In such a case, the heterotic string on the stack is not well-defined. In an ordinary orbifold, this is the

${ }^{20}$ Fock vacua have been much more commonly described in terms of sections of bundles over CFT moduli spaces, see e.g. [94, 95, but descriptions as sections of bundles over target spaces are much more rare.

${ }^{21}$ Since the $\alpha$-sector has components which are $t_{\alpha}$ gerbes, $t_{\alpha}$-th roots of bundles might exist, though not necessarily. (See Appendix D for examples of bundles on $\mathbb{Z}_{n}$-gerbes which do and do not admit $n$th roots.) 
case that the Fock vacua (and hence perturbative states built from them) form a merely projective representation of the orbifold group, instead of an honest representation, and the projection operator built implicitly in the structure of the string one-loop partition function no longer functions. This condition represents a new (to our knowledge) consistency condition, so let us take a few paragraphs to elaborate on this point.

At least morally, this condition is a generalization to stacks of the old requirement that " $c_{1} \equiv 0 \bmod 2$ " for bundles embedded in $E_{8}$ in the standard fashion. That constraint could be understood in two ways:

- In low-energy supergravity, this is ultimately the statement that the $U(n)$ bundle can be lifted to $\operatorname{Spin}(16)$, realized by the left GSO projection, whose embeddeding into $E_{8}$ then factors through $\operatorname{Spin}(16) / \mathbb{Z}_{2}$,

- On the worldsheet, this is the statement that the Fock vacua are welldefined in a left $\mathrm{R}$ sector. The Fock vacua couple to a square root of the gauge bundle; that square root will exist if and only if " $c_{1} \equiv 0 \bmod$ 2."

(For another recent discussion of constraints of this form, see for example [28.)

In toroidal orbifolds, this constraint is very mild, but illustrates an important point: not only the bundle must admit a square root, but also the equivariant structure. For a typical toroidal orbifold, the bundle factors are all trivial, only the equivariant structures are nontrivial. In typical such orbifolds, $K_{\alpha}$ is the trivial line bundle with trivial connection, but although $\operatorname{det} \mathcal{E}_{0}^{\alpha}$ is a trivial bundle, the equivariant structure may be nontrivial. In left $\mathrm{R}$ sector, $\mathcal{E}_{0}^{\alpha}$ describes couples to fermions that are both periodic and invariant under the orbifold group, so the equivariant structure is trivial. In a left NS sector, on the other hand, $\mathcal{E}_{0}^{\alpha}$ describes periodic fermions, which are anti-invariant under the orbifold group. In a left NS sector, if the rank of $\mathcal{E}_{0}^{\alpha}$ is even, the induced equivariant structure on $\operatorname{det} \mathcal{E}_{0}^{\alpha}$ is trivial; if the rank of $\mathcal{E}_{0}^{\alpha}$ is odd, then the induced equivariant structure is nontrivial, and does not admit a square root, hence there is an obstruction to the existence of the orbifold in this case.

We can build an example of a toroidal orbifold in which this condition appears nontrivially as follows. Consider an $E_{8} \times E_{8}$ string on a $\left[T^{4} / \mathbb{Z}_{6}\right]$ orbifold, in which the generator $g$ of $\mathbb{Z}_{6}$ act on $T^{4}$ by multiplication by -1 . Define a rank 4 bundle over this stack by taking $\mathcal{O}^{\oplus 4}$ over $T^{4}$, and let $g$ act 
with eigenvalues

$$
(\exp (6 \pi i / 6)=-1, \exp (4 \pi i / 6)=\exp (2 \pi i / 3), \exp (2 \pi i / 3), \exp (-2 \pi i / 6))
$$

It is straightforward to check that this satisfies level-matching, in the sense of [59]. In the $g$-twisted left NS sector, there will be one periodic fermion, which is problematic as above.

It is tempting to speculate that a necessary condition for the existence of the square root A.3 can be written in the form

$$
c_{1}^{\mathrm{rep}}(\mathcal{E}) \equiv c_{1}^{\mathrm{rep}}(T \mathfrak{X}) \bmod 2
$$

applying the Chern-rep's discussed in Sections 5.6 and Appendix C. We will leave such an interpretation to future work.

\section{A.5. Spectrum result and Serre duality}

Finally, we are ready to associate sheaf cohomology groups to elements of the spectrum. A general element of the spectrum will have the form

$$
\lambda_{-}^{a_{1}} \cdots \lambda_{-}^{a_{m}} \psi_{+}^{\bar{\tau}_{1}} \cdots \psi_{+}^{\bar{\tau}_{k}}|0\rangle
$$

where each $\lambda$ and $\psi$ has some unspecified moding, such that the sum of the modings equals the vacuum energy computed earlier. Canonical commutation relations descend to statements of the form

$$
\left\{\lambda_{p}^{a}, \lambda_{-p}^{\bar{b}}\right\} \propto h^{a \bar{b}}, \quad\left\{\psi_{p}^{i}, \psi_{-p}^{\bar{\jmath}}\right\} \propto g^{i \bar{\jmath}}
$$

where $p$ is a moding. So long as the modings are all negative, both holomorphic and antiholomorphic-indexed fermions can appear in states. For zero modes, our Fock vacuum conventions are such that only $\lambda_{-, 0}^{\bar{a}}$ and $\psi_{+, 0}^{i}$ contribute.

In any event, it should now be clear, following [65], that on component $\alpha$, states of the form 22

$$
\prod_{n}\left(\lambda_{-, n}^{a_{1}} \cdots \lambda_{-, n}^{a_{m_{n}}} \lambda_{-, n}^{\bar{b}_{1}} \cdots \lambda_{-, n}^{\bar{b}_{p_{n}}} \psi_{+, n}^{j_{1}} \cdots \psi_{+, n}^{j_{\ell_{n}}} \psi_{+, n}^{\bar{\tau}_{1}} \cdots \psi_{+, n}^{\bar{\imath}_{k_{n}}}\right)|0\rangle
$$

${ }^{22}$ We have omitted modings for reasons of notational sanity. 
(where the fermion modings add up to the vacuum energy in the $\alpha$ sector) are counted by the sheaf cohomology group

$$
H^{k_{0}}\left(\left.I_{\mathfrak{X}}\right|_{\alpha},\left(\wedge^{m_{0}} \mathcal{E}_{0}^{\alpha *}\right) \otimes_{n>0}\left(\wedge^{m_{n}} \mathcal{E}_{n}^{\alpha} \otimes \wedge^{p_{n}} \mathcal{E}_{n}^{\alpha *} \otimes \wedge^{\ell_{n}} T_{n}^{\alpha} \otimes \wedge^{k_{n}} T_{n}^{\alpha *}\right) \otimes \mathcal{F}\right)
$$

where

$$
\mathcal{F}^{\alpha}=\sqrt{K_{\alpha} \otimes \operatorname{det} \mathcal{E}_{0}^{\alpha}} \otimes_{n>0}\left(\left(\operatorname{det} \mathcal{E}_{n}^{\alpha}\right)\left(\operatorname{det} T_{n}^{\alpha}\right)^{-1}\right)^{-\frac{n}{t_{\alpha}}-\left[-\frac{n}{t_{\alpha}}\right]-\frac{1}{2}}
$$

(reflecting the Fock vacuum). Strictly speaking, not all states need be of the form above - for example, one might also be able to multiply in bosonic $\partial \phi$ modes. As their inclusion is standard and their treatment should now be clear, for reasons of brevity we shall move on.

For example, if $\left.I_{\mathfrak{X}}\right|_{\alpha}=\left[\right.$ point $\left./ \mathbb{Z}_{2}\right]$, then this becomes

$$
H^{k_{0}}\left(\text { point },\left(\wedge^{m_{0}} \mathcal{E}_{0}^{\alpha *}\right) \otimes_{n>0}\left(\wedge^{m_{n}} \mathcal{E}_{n}^{\alpha *} \otimes \wedge^{p_{n}} \mathcal{E}_{n}^{\alpha *} \otimes \wedge^{\ell_{n}} T_{n}^{\alpha} \otimes \wedge^{k_{n}} T_{n}^{\alpha *}\right) \otimes \mathcal{F}^{\alpha}\right)^{\mathbb{Z}_{2}}
$$

(Taking group invariants is encoded implicitly in taking sheaf cohomology on the quotient stack.) This group vanishes if $k_{0} \neq 0$, and when $k_{0}=0$, is the dimension of the $\mathbb{Z}_{2}$-invariant part of the vector space fibers.

Finally, in a physical computation, one must impose the left- and rightGSO projections. For states of the form above, this will amount to a chirality constraint on $k_{0}$ and $m_{0}$. As the procedure is standard, we will say no more.

One of the central observations of the heterotic spectrum computation on smooth manifolds in [65] is that it is closed under Serre duality. The same is true here. First, for any component of the inertia stack indexed by an automorphism $\alpha$, there is another (not necessarily distinct) component indexed by $\alpha^{-1}$, which is isomorphic:

$$
\left.\left.I_{\mathfrak{X}}\right|_{\alpha} \cong I_{\mathfrak{X}}\right|_{\alpha^{-1}}
$$

Eigenbundle decompositions are closely related:

$$
\begin{aligned}
& T_{n}^{\alpha^{-1}} \cong T_{-n}^{\alpha}, \quad T_{0}^{\alpha^{-1}} \cong T_{0}^{\alpha} \\
& \mathcal{E}_{n}^{\alpha^{-1}} \cong \mathcal{E}_{-n}^{\alpha}, \quad \mathcal{E}_{0}^{\alpha^{-1}} \cong \mathcal{E}_{0}^{\alpha}
\end{aligned}
$$

(in conventions where $-n$ denotes the component associated to the character of the inverse). Let us now consider the following factor in the Fock vacuum 
bundle,

$$
\mathcal{F}_{+}^{\alpha}=\otimes_{n>0}\left(\left(\operatorname{det} \mathcal{E}_{n}^{\alpha}\right)\left(\operatorname{det} T_{n}^{\alpha}\right)^{-1}\right)^{-\frac{n}{t_{\alpha}}-\left[-\frac{n}{t_{\alpha}}\right]-\frac{1}{2}}
$$

(where the tensor product runs over all nontrivial representations of $\mathbb{Z}_{t^{\alpha}}$ ). Using relations such as $\mathcal{E}_{n}^{\alpha^{-1}} \cong \mathcal{E}_{-n}^{\alpha}$, we see that each factor in $\mathcal{F}_{+}^{\alpha^{-1}}$ is equivalent to a factor in $\mathcal{F}_{+}^{\alpha}$, but with an exponent of the opposite sign, hence

$$
\mathcal{F}_{+}^{\alpha^{-1}} \cong\left(\mathcal{F}_{+}^{\alpha}\right)^{*}
$$

As the combinatorics in these exponents is slightly complicated, let us consider some special cases to explicitly confirm this prediction. When $t_{\alpha}=2$,

$$
\begin{aligned}
\mathcal{F}_{+}^{\alpha} & =\left(\left(\operatorname{det} \mathcal{E}_{1}^{\alpha}\right)\left(\operatorname{det} T_{1}^{\alpha}\right)^{-1}\right)^{-\frac{1}{2}-\left[-\frac{1}{2}\right]-\frac{1}{2}} \\
& =\left(\left(\operatorname{det} \mathcal{E}_{1}^{\alpha}\right)\left(\operatorname{det} T_{1}^{\alpha}\right)^{-1}\right)^{0} \cong \mathcal{O} \cong\left(\mathcal{F}_{+}^{\alpha^{-1}}\right)^{*}
\end{aligned}
$$

When $t_{\alpha}=3$,

$$
\begin{aligned}
\mathcal{F}_{+}^{\alpha} & =\left(\left(\operatorname{det} \mathcal{E}_{1}^{\alpha}\right)\left(\operatorname{det} T_{1}^{\alpha}\right)^{-1}\right)^{-\frac{1}{3}-\left[-\frac{1}{3}\right]-\frac{1}{2}} \otimes\left(\left(\operatorname{det} \mathcal{E}_{2}^{\alpha}\right)\left(\operatorname{det} T_{2}^{\alpha}\right)^{-1}\right)^{-\frac{2}{3}-\left[-\frac{2}{3}\right]-\frac{1}{2}} \\
& =\left(\left(\operatorname{det} \mathcal{E}_{1}^{\alpha}\right)\left(\operatorname{det} T_{1}^{\alpha}\right)^{-1}\right)^{+1 / 6} \otimes\left(\left(\operatorname{det} \mathcal{E}_{2}^{\alpha}\right)\left(\operatorname{det} T_{2}^{\alpha}\right)^{-1}\right)^{-1 / 6}
\end{aligned}
$$

and

$$
\begin{aligned}
\mathcal{F}_{+}^{\alpha^{-1}} & =\left(\left(\operatorname{det} \mathcal{E}_{1}^{\alpha^{-1}}\right)\left(\operatorname{det} T_{1}^{\alpha^{-1}}\right)^{-1}\right)^{+1 / 6} \otimes\left(\left(\operatorname{det} \mathcal{E}_{2}^{\alpha^{-1}}\right)\left(\operatorname{det} T_{2}^{\alpha^{-1}}\right)^{-1}\right)^{-1 / 6} \\
& =\left(\left(\operatorname{det} \mathcal{E}_{2}^{\alpha}\right)\left(\operatorname{det} T_{2}^{\alpha}\right)^{-1}\right)^{+1 / 6} \otimes\left(\left(\operatorname{det} \mathcal{E}_{1}^{\alpha}\right)\left(\operatorname{det} T_{1}^{\alpha}\right)^{-1}\right)^{-1 / 6} \\
& =\left(\mathcal{F}_{+}^{\alpha}\right)^{*}
\end{aligned}
$$

In this fashion we confirm equation A.5 explicitly.

Vacuum energies are invariant: if a fermion boundary condition in sector $\alpha$ is determined by $\theta$, then in $\alpha^{-1}$ it is determined by $-\theta$, but vacuum energies only depend upon $(\theta)^{2}$, and so are invariant. Contributions to the spectrum from sector $\alpha$ are matched by Serre duals in sector $\alpha^{-1}$. In terms of global quotients by finite groups, this means the untwisted sector closes into itself under Serre duality, but twisted sectors are exchanged. For example, 
the Serre duals to A.4 are given by

$$
\begin{aligned}
H^{\operatorname{dim}-k_{0}}\left(\left.I_{\mathfrak{X}}\right|_{\alpha},\left(\wedge^{m_{0}} \mathcal{E}_{0}^{\alpha}\right) \otimes_{n>0}\left(\wedge^{m_{n}} \mathcal{E}_{n}^{\alpha *} \otimes \wedge^{p_{n}} \mathcal{E}_{n}^{\alpha} \otimes \wedge^{\ell_{n}} T_{n}^{\alpha *} \otimes \wedge^{k_{n}} T_{n}^{\alpha}\right)\right. \\
\otimes\left(\mathcal{F}_{+}^{\alpha}\right)^{*} \otimes \sqrt{\left.K_{\alpha}^{*} \otimes \operatorname{det} \mathcal{E}_{0}^{\alpha *} \otimes K_{\alpha}\right)^{*}} \\
=H^{\operatorname{dim}-k_{0}}\left(\left.I_{\mathfrak{X}}\right|_{\alpha^{-1}},\left(\wedge^{\mathrm{rk}-m_{0}} \mathcal{E}_{0}^{\alpha^{-1}}\right)\right. \\
\otimes_{n>0}\left(\wedge^{m_{n}} \mathcal{E}_{n}^{\alpha^{-1} *} \otimes \wedge^{p_{n}} \mathcal{E}_{n}^{\alpha^{-1}} \otimes \wedge^{\ell_{n}} T_{n}^{\alpha^{-1} *} \otimes \wedge^{k_{n}} T_{n}^{\alpha^{-1}}\right) \\
\left.\otimes \mathcal{F}_{+}^{\alpha^{-1}} \otimes \sqrt{K_{\alpha^{-1}} \otimes \operatorname{det} \mathcal{E}_{0}^{\alpha^{-1}}}\right)^{*},
\end{aligned}
$$

which is of the same form as equation A.4, as desired. Note that the Fock vacuum contribution is essential for the spectrum to close under Serre duality in this fashion: otherwise, Serre duality would generate a factor of $K_{\alpha}$ in the coefficients, unmatched by anything else, and which is nontrivial if the $\alpha$ component is not Calabi-Yau23. Our computations so far have focused on the $(\mathrm{R}, \mathrm{R})$ sector, but one should note that identical considerations hold in the (NS, R) sector as well.

In the special case that the stack $\mathfrak{X}$ is a smooth Calabi-Yau manifold $X$, these computational methods reduce to those of [65]. In this case, the inertia stack $I_{\mathfrak{X}}$ has no nontrivial components: $I_{\mathfrak{X}}=X$. Furthermore, we typically take $\operatorname{det} \mathcal{E}$ to be trivial, so the Fock vacuum is a section of a trivial line bundle.

In the special case that the stack $\mathfrak{X}$ is a toroidal orbifold, again these methods reduce to known results. In this case, all of the bundles involved are trivial, so sheaf cohomology is nontrivial only in degree zero, and sheaf cohomology on a stack just takes group invariants of the coefficients.

A less trivial example is discussed in Section 5.3. Further examples and computational techniques will appear in [92].

Just as in 65, in principle the number of generations can be computed as an index based on the spectrum. We shall not work through details here, but Appendix C contains general results on index theory computations on stacks.

23 To make it clear that this condition is nontrivial, here is an example of a global orbifold in which a twisted sector has support on a non-Calabi-Yau subvariety. Let $X$ be a branched double cover of $\mathbb{P}^{n}$, branched over a degree $2 n+2$ locus. Now, orbifold by the globally-acting $\mathbb{Z}_{2}$ that exchanges the sheets of the cover. This leaves invariant the degree $2 n+2$ branch locus, which is not Calabi-Yau. 


\section{A.6. A/2 model spectra}

In this appendix we have focused on physical heterotic string spectra. It is possible to apply the same methods to the $\mathrm{A} / 2$ model to formulate a mathematical theory of sheaf cohomology of orbifolds, and this has been done in $[92$.

Briefly, the A/2 model is a heterotic analogue of the A model topological field theory. If $X$ is a smooth space and $\mathcal{E} \rightarrow X$ a holomorphic vector bundle, then the $\mathrm{A} / 2$ model is well-defined if both 24

$$
\operatorname{ch}_{2}(\mathcal{E})=\operatorname{ch}_{2}(T X) \text { and } \operatorname{det} \mathcal{E}^{*} \cong K_{X}
$$

See e.g. 99 107] for more information on the A/2 and B/2 models. As this is no longer a physical theory, constraints on the dimension of $X$ and rank of $\mathcal{E}$ are dropped. When $X$ is smooth, the massless spectrum consists of sheaf cohomology groups of the form

$$
H^{\bullet}\left(X, \wedge \bullet \mathcal{E}^{*}\right)
$$

When $X$ is a stack $\mathfrak{X}$, reference [92] applies methods similar to those in this appendix (modulo restricting to $(\mathrm{R}, \mathrm{R}$ ) sector states and omitting the GSO projections) to define a generalization, which broadly speaking adds in various sheaf cohomology groups associated to twisted sectors (nontrivial components of the inertia stack).

\section{Appendix B. Line bundles on gerbes over projective spaces}

For any stack $\mathfrak{X}$ presented as $\mathfrak{X}=[X / G]$ for some space $X$ and group $G$, a vector bundle (sheaf) on $\mathfrak{X}$ is the same as a $G$-equivariant vector bundle

24 The second condition arises from the need to make the path integral measure a scalar, ultimately. On stacks, one might wonder whether one should impose an analogous condition in each individual twisted sector, something of the form

$$
\operatorname{det} \mathcal{E}_{0}^{\alpha *} \cong K_{\alpha}
$$

Reference 92] does not impose a stronger condition of this sort. One reason is that there is no analogue of such a condition in GLSM's (whereas the original condition $\operatorname{det} \mathcal{E}^{*} \cong K_{\mathfrak{X}}$ on the entire stack does manifest in GLSM's). In terms of making sense of path integral measures, in twisted sectors one must insert twist fields to get nonzero results, and which would modify any such constraint one wished to impose on individual twisted sectors. 
(sheaf) on $X$. Suppose that $G$ is an extension

$$
1 \longrightarrow K \longrightarrow G \longrightarrow H \longrightarrow 1
$$

where $K$ acts trivially on $X$, and $G / K \cong H$ acts effectively. In this case, $\mathfrak{X}=[X / G]$ is a $K$-gerbe. A vector bundle on $\mathfrak{X}$ is a $G$-equivariant vector bundle on $X$, and as such, the $K$ action is defined by a representation of $K$ on the fibers of that vector bundle.

In this section, we will discuss in greater detail the special case of line bundles on gerbes over projective spaces.

\section{B.1. Generalities}

Let us first review some basic properties of line bundles on gerbes over projective spaces, and then we will outline their sheaf cohomology.

First, let us consider some simple explicit examples. The total space of the line bundle $\mathcal{O}(-m)$ over the projective space $\mathbb{P}^{n}$ can be described ${ }^{25}$ by a gauged linear sigma model with fields of $U(1)$ charges

$$
\begin{array}{cccc}
x_{1} & \cdots & x_{n+1} & p \\
\hline 1 & \cdots & 1 & -m
\end{array}
$$

Now, a $\mathbb{Z}_{k}$ gerbe over $\mathbb{P}^{n}$ can be described by a gauged linear sigma model in which the $n+1$ fields/homogeneous coordinates have weight $k$ instead of weight 1 , as discussed in e.g. [16]. Then, for example, the GLSM with fields and $U(1)$ charges

$$
\begin{array}{cccc}
x_{1} & \cdots & x_{n+1} & p \\
\hline k & \cdots & k & -k
\end{array}
$$

is surely going to be the pullback of $\mathcal{O}(-1) \rightarrow \mathbb{P}^{n}$ to the gerbe.

However, how does one interpret GLSM's defined by, for example:

$$
\begin{array}{cccc}
x_{1} & \cdots & x_{n+1} & p \\
\hline k & \cdots & k & -1
\end{array}
$$

\footnotetext{
${ }^{25}$ For $m>0$. The total spaces of line bundles of positive degree over projective spaces do not seem to admit a GLSM description, even though they are toric varieties - they can be described as GIT quotients of open subsets of $\mathbb{C}^{n+2}$ by $\mathbb{C}^{\times}$, but not as a GIT quotient of the full complex vector space, and they naturally compactify to $\mathbb{P}_{[1, \ldots, 1, m]}^{n+1}$. We would like to thank D. Skinner for asking a question that made this manifest.
} 
This is the total space of what is sometimes referred to as the "OOO $(1 / k)$ " line bundle over the $\mathbb{Z}_{k}$ gerbe $\mathbb{P}_{[k, \ldots, k]}^{n}$. It is an example of a line bundle on the gerbe that is not a pullback of a line bundle on the base space - the gerbe has more bundles than the base space. More to the point, it can only be understood as the total space of a line bundle on a gerbe - so a physicist who was very careful in a study of GLSM's would eventually be forced to discover gerbes in order to make sense of this example.

In addition to being a line bundle over the stack, the total space of the $\mathcal{O}(1 / k)$ line bundle is also a fibered orbifold over the projective space $\mathbb{P}^{n}$ - it is a type of fiber bundle over $\mathbb{P}^{n}$, in which the fibers are the orbifolds $\left[\mathbb{C} / \mathbb{Z}_{k}\right]$. For this reason, these are sometimes known as 'orbibundles;' see e.g. [108] for references to the literature under this name. (This same structure has also been discussed in connection with interpreting hybrid Landau-Ginzburg models, see e.g. [109].)

Not all $\mathbb{Z}_{k}$ gerbes on projective spaces are of the form of weighted projective stacks. A more general class was discussed in e.g. [16] [Section 3.3], and, roughly, are given by $\mathbb{C}^{\times}$quotients of principal $\mathbb{C}^{\times}$bundles over $\mathbb{P}^{n}$. Specifically, consider a GLSM with fields $x_{i}, z$, and two $\mathbb{C}^{\times}$actions, as follows:

\begin{tabular}{c|cc} 
& $x_{i}$ & $z$ \\
\hline$\lambda$ & 1 & $-n$ \\
$\mu$ & 0 & $k$
\end{tabular}

The first $\mathbb{C}^{\times}, \lambda$, defines the total space of a line bundle on $\mathbb{P}^{n}$ of degree $-n$. The second $\mathbb{C}^{\times}, \mu$, quotients out the fibers, leaving a $\mathbb{Z}_{k}$ kernel. The result is a $\mathbb{Z}_{k}$ gerbe over $\mathbb{P}^{n}$, of characteristic class $-n \bmod k$. The weighted projective stacks we have been discussing correspond to an alternative presentation in the special case that $n=1$. One can define line bundles over these gerbes in the obvious fashion.

The notation $\mathcal{O}(1 / k)$, while initially catchy, is unfortunately ambiguous - for example, it does not distinguish a twisted bundle of $c_{1}=k$ over the gerbe from the pullback from $\mathbb{P}^{n}$ of an ordinary line bundle of $c_{1}=1$. Let us introduce a more precise notation.

We will use " $\mathcal{O}_{\Lambda}(m)$ " to denote a line bundle defined by a superfield of charge $m$. For bundles on, say, ordinary projective spaces, the $k=1$ case, a superfield of charge $m$ couples to the line bundle $\mathcal{O}(m)$.

To understand the meaning of this notation, let us first consider a $\mathbb{Z}_{2}$ gerbe over $\mathbb{P}^{n}$ defined by the weighted projective stack $\mathbb{P}_{[2,2, \ldots, 2]}^{n}$. Let $G \mathbb{P}^{n}$ denote the gerbe, and $\pi: G \mathbb{P}^{n} \rightarrow \mathbb{P}^{n}$ the natural projection from the gerbe onto the underlying projective space. 
Now, coherent sheaves on the gerbe decompose into twisted sheaves on the underlying space (see Section 2.3 or [17]). Formally, if $\alpha \in H^{2}\left(\mathbb{P}^{n}, \mathbb{Z}_{2}\right)$ is the characteristic class of the gerbe, then

$$
\operatorname{Coh}\left(G \mathbb{P}^{n}\right)=\operatorname{Coh}\left(\mathbb{P}^{n}, 1(\alpha)\right) \cup \operatorname{Coh}\left(\mathbb{P}^{n}, \chi(\alpha)\right)
$$

where $\operatorname{Coh}(X, \lambda)$ denotes coherent sheaves on $X$ twisted by a 2-cocycle $\lambda$. In the notation above, 1 and $\chi$ are the two irreducible representations of $\mathbb{Z}_{2}$, so $1(\alpha)$ is the vanishing 2 -cocycle and $\chi(\alpha)$ is a cocycle that does not vanish identically. Note that both cocycles are cohomologous to the identity both components of $\operatorname{Coh}\left(G \mathbb{P}^{n}\right)$ are isomorphic to ordinary coherent sheaves $\operatorname{Coh}\left(\mathbb{P}^{n}\right)$. (This resolves a potential contradiction, in that the rank of a bundle twisted by a cohomologically nontrivial cocycle, must be divisible by the order of the cocycle, and so here would need to be divisible by $k$ - truly twisted line bundles do not exist.)

In this language, we can immediately read off that

$$
\mathcal{O}_{\Lambda}(k)= \begin{cases}\operatorname{Coh}\left(\mathbb{P}^{n}, 1(\alpha)\right) & k \text { even } \\ \operatorname{Coh}\left(\mathbb{P}^{n}, \chi(\alpha)\right) & k \text { odd }\end{cases}
$$

In other words, if $k$ is even, then $\mathcal{O}_{\Lambda}(k)$ is a pullback to the gerbe from a line bundle on the base. For other values of $k$, the bundle is twisted by an action of the $\mathbb{Z}_{2}$.

Now, the projection map $\pi: G \mathbb{P}^{n} \rightarrow \mathbb{P}^{n}$ defines a functor

$$
\pi^{*}: \operatorname{Coh}\left(\mathbb{P}^{n}\right) \stackrel{\sim}{\longrightarrow} \operatorname{Coh}\left(\mathbb{P}^{n}, 1(\alpha)\right)
$$

In addition, there is another functor

$$
\pi_{1}^{*} \equiv \pi^{*} \otimes \mathcal{O}_{\Lambda}(1): \operatorname{Coh}\left(\mathbb{P}^{n}\right) \stackrel{\sim}{\longrightarrow} \operatorname{Coh}\left(\mathbb{P}^{n}, \chi(\alpha)\right)
$$

(In fact, there is an analogue of $\pi_{1}^{*}$ for every $\mathcal{O}_{\Lambda}$ (odd).)

To determine $\pi^{*} \mathcal{O}(m)$ in terms of $\mathcal{O}_{\Lambda}$ 's, consider the commutative diagram

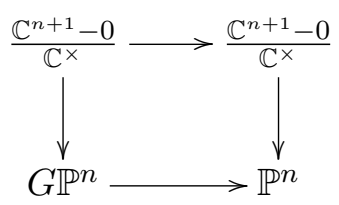


The line bundle $\mathcal{O}(k)$, defined by weights $1, \ldots, 1, k$, pulls back to weights $2, \ldots, 2,2 k$, from which we deduce that

$$
\pi^{*} \mathcal{O}(k)=\mathcal{O}_{\Lambda}(2 k)
$$

which implies

$$
\pi_{1}^{*} \mathcal{O}(k)=\mathcal{O}_{\Lambda}(2 k+1)
$$

Note that although $\pi^{*}$ preserves tensor products, $\pi_{1}^{*}$ does not preserve tensor products:

$$
\begin{aligned}
\pi_{1}^{*}(\mathcal{O}(k) \otimes \mathcal{O}(m)) & \cong \pi_{1}^{*} \mathcal{O}(k+m) \\
& \cong \mathcal{O}_{\Lambda}(2 k+2 m+1) \\
& ¥ \mathcal{O}_{\Lambda}(2 k+2 m+2) \cong\left(\pi_{1}^{*} \mathcal{O}(k)\right) \otimes\left(\pi_{1}^{*} \mathcal{O}(m)\right)
\end{aligned}
$$

Indeed, this is an immediate consequence of the definition of $\pi_{1}^{*}$. In addition, for the same reason, $\pi_{1}^{*}$ does not commute with duality of bundles

$$
\pi_{1}^{*}\left(\mathcal{L}^{\vee}\right) \approx\left(\pi_{1}^{*} \mathcal{L}\right)^{\vee}
$$

Now, for any finite gerbe over any space, the tangent bundle of the gerbe is just the pullback (under $\pi$ ) of the tangent bundle to the space. One way to see this is to work locally on the atlas, which is just a finite cover, and so the tangent bundle should be the same. We can see this explicitly in the present case as follows. For the $\mathbb{Z}_{2}$ gerbe $G \mathbb{P}^{n}=\mathbb{P}_{[2, \ldots, 2]}^{n}$, the tangent bundle seen by the gauged linear sigma model is

$$
0 \longrightarrow \mathcal{O}_{\Lambda} \longrightarrow \mathcal{O}_{\Lambda}(2)^{n+1} \longrightarrow T G \mathbb{P}^{n} \longrightarrow 0
$$

Using the isomorphisms above, we see this short exact sequence is the same as

$$
0 \longrightarrow \pi^{*} \mathcal{O} \longrightarrow \pi^{*} \mathcal{O}(1)^{n+1} \longrightarrow T G \mathbb{P}^{n} \longrightarrow 0
$$

which is just $\pi^{*}$ of the Euler sequence for the tangent bundle

$$
0 \longrightarrow \mathcal{O} \longrightarrow \mathcal{O}(1)^{n+1} \longrightarrow T \mathbb{P}^{n} \longrightarrow 0
$$

For $\mathbb{Z}_{k}$ gerbes over $\mathbb{P}^{n}$ built as the weighted projective stack $\mathbb{P}_{[k, \ldots, k]}^{n}$, there is a closely analogous story. Here, coherent sheaves on $G \mathbb{P}^{n}$ decompose as

$$
\operatorname{Coh}\left(G \mathbb{P}^{n}\right)=\cup_{\chi} \operatorname{Coh}\left(\mathbb{P}^{n}, \chi(\alpha)\right)
$$


where the union is over irreducible representations of $\mathbb{Z}_{k}$, and there are $k$ different pullbacks, first the canonical

$$
\pi^{*}: \operatorname{Coh}\left(\mathbb{P}^{n}\right) \stackrel{\sim}{\longrightarrow} \operatorname{Coh}\left(\mathbb{P}^{n}, 1(\alpha)\right)
$$

followed by $\pi_{i}^{*}(-) \equiv \pi^{*}(-) \otimes \mathcal{O}_{\Lambda}(i)$. Identifying $\pi_{0}^{*}$ with $\pi^{*}$, we have the general relation

$$
\pi_{i}^{*} \mathcal{O}(m)=\mathcal{O}_{\Lambda}(k m+i)
$$

An argument nearly identical to the one above shows that the tangent bundle $T G \mathbb{P}^{n}$ seen by a gauged linear sigma model is given by $\pi^{*} T \mathbb{P}^{n}$, exactly as must be true on general grounds.

\section{B.2. Sheaf cohomology}

On a global quotient stack $\mathfrak{X}=[X / G]$, for $G$ finite, given a vector bundle $\mathcal{E} \rightarrow \mathfrak{X}$, (equivalently, a $G$-equivariant bundle on $X$,)

$$
H^{\bullet}(\mathfrak{X}, \mathcal{E})=H^{\bullet}(X, \mathcal{E})^{G}
$$

In our discussion of massless spectra of heterotic strings on stacks, this is ultimately the reason why in orbifolds one gets $G$-invariants.

Now, nontrivial gerbes over projective spaces have a global quotient description as some $[X / G]$ for $G$ nonfinite, and the simple description of sheaf cohomology above in terms of $G$-invariants is only valid for $G$ finite, so for general cases a different approach is required. For example, let $\mathfrak{X}=\mathbb{P}_{[k, \ldots, k]}^{n}$, and $\mathcal{O}_{\mathfrak{X}}(m)$ as above, then

$$
H^{i}\left(\mathfrak{X}, \mathcal{O}_{\mathfrak{X}}(m)\right)= \begin{cases}0 & k \nmid m, \\ H^{i}\left(\mathbb{P}^{n}, \mathcal{O}_{\mathbb{P}^{n}}(m / k)\right) & k \mid m .\end{cases}
$$

For $m \geq 0$, we can check this as follows. First,

$$
H^{i}\left(\mathfrak{X}, \mathcal{O}_{\mathfrak{X}}(m)\right)=H_{\mathbb{C}^{\times}}^{i}\left(\mathbb{C}^{n+1}-\{0\}, \mathcal{O}\right),
$$

where the $\mathcal{O}$ coefficients have weight $m$ under the $\mathbb{C}^{\times}$. In principle, there is a spectral sequence converging to the right-hand side, with level-two terms

$$
H^{p}\left(\mathbb{C}^{\times}, H^{q}\left(\mathbb{C}^{n+1}-\{0\}, \mathcal{O}\right)\right)
$$

but $H^{q}\left(\mathbb{C}^{n+1}-\{0\}, \mathcal{O}\right)=0$ for $q \neq 0, n$, and

$$
H^{0}\left(\mathbb{C}^{n+1}-\{0\}, \mathcal{O}\right)=\mathbb{C}\left[x_{0}, \ldots, x_{n}\right] .
$$


(The degree $n$ cohomology is also nonzero and infinite-dimensional, but it will not contribute any invariants for $m \geq 0$, only for $m<0$, so we omit it from this discussion.) For $\lambda \in \mathbb{C}^{\times}$, the representation

$$
\rho: \mathbb{C}^{\times} \longrightarrow \mathrm{GL}\left(\mathbb{C}\left[x_{0}, \ldots, x_{n}\right]\right)
$$

is defined by

$$
\rho_{\lambda}(f(x))=\lambda^{-m} f\left(\lambda^{k} x_{0}, \ldots, \lambda^{k} x_{n}\right) .
$$

The group $H^{p}\left(\mathbb{C}^{\times},\left(\mathbb{C}\left[x_{0}, \ldots, x_{n}\right], \rho\right)\right)$ is zero unless $p=0$, since it is a reductive group, and for $p=0$ is given by the invariants.

Next, let us compute the invariants. Decompose

$$
f=f_{0}+\cdots+f_{N}
$$

where $f_{d}$ denotes a homogeneous polynomial of degree $d$. Under the $\mathbb{C}^{\times}$ action,

$$
\rho_{\lambda}(f)=\lambda^{-m} f_{0}+\lambda^{-m+k} f_{1}+\cdots+\lambda^{-m+k N} f_{N} .
$$

Thus, $\mathbb{C}^{\times}$invariants only exist in the case that $k$ divides $m$, and in that case, are counted by degree $m / k$ polynomials in $n+1$ variables.

Now, let us compare to the original claim. It is a standard result that for $\ell>0$,

$$
H^{i}\left(\mathbb{P}^{n}, \mathcal{O}_{\mathbb{P}^{n}}(\ell)\right)= \begin{cases}0 & i \neq 0 \\ \operatorname{Sym}^{\ell} \mathbb{C}^{n+1} & i=0 .\end{cases}
$$

In other words, the only nonzero cohomology is in degree zero, and in that degree, it is counted by homogeneous polynomials of degree $\ell$ in $n+1$ variables. The desired result follows.

\section{Appendix C. Chern classes on the inertia stack}

As we are manipulating bundles on stacks, it is worth spending a little time reviewing corresponding Chern classes. It is possible to define Chern classes on a stack itself; for example, Chern classes of a vector bundle $\mathcal{E}$ on a quotient stack $[X / G]$ are simply $G$-equivariant Chern classes of $\mathcal{E}$ on $X$. However, these Chern classes do not always behave well under mathematical manipulations, and in any event a different notion of Chern classes and Chern characters, denoted $c^{\text {rep }}$ and $\mathrm{ch}^{\mathrm{rep}}$, exists and is relevant for index theory. These alternative notions of Chern classes do not live in the cohomology of the original stack, but rather of the inertia stack, which encodes 
twisted sectors of string orbifolds. (See Appendix A for more information on the inertia stack.)

In this section, we will illustrate how to compute such Chern classes and characters (denoted $c^{\text {rep }}$ and $\mathrm{ch}^{\text {rep}}$ ) and describe their appearance in index theory in some examples. It is tempting to wonder whether one could derive extra anomaly constraints on orbifolds from these stack Chern classes over nontrivial components of the inertia stack, but we argue that does not seem to happen in heterotic compactifications in Section 5.6 (though see Section A.4 for a possible application of $c_{1}^{\text {rep }}$ ).

For any stack $\mathfrak{X}$, let $V$ be a vector bundle over $\mathfrak{X}$, and $I_{\mathfrak{X}}$ the inertia stack of $\mathfrak{X}$. Let $q: I_{\mathfrak{X}} \rightarrow \mathfrak{X}$ denote the natural projection operator onto one component.

We define Chern classes of $V$ as follows. First, pullback $V$ to $I_{\mathfrak{X}}$ along $q$. Then, on each component $\alpha$ of $I_{\mathfrak{X}}, q^{*} V$ will decompose into eigenbundles of the action of the stabilizer for that component:

$$
\left.q^{*} V\right|_{\alpha}=\oplus_{\chi} V_{\alpha, \chi}
$$

(When $\alpha$ is the identity, our conventions are that there is only one component, associated to the trivial character.) Define $\operatorname{ch}^{\mathrm{rep}}(V)$ over a component $\alpha$ to be

$$
\left.\operatorname{ch}^{\mathrm{rep}}(V)\right|_{\alpha} \equiv \bigoplus_{\chi} \operatorname{ch}\left(V_{\alpha, \chi}\right) \otimes \chi,
$$

where $\chi$ is the eigenvalue of that component of $q^{*} V$ under the stabilizer, and ch denotes the naive notion of Chern classes, living in equivariant cohomology pertinent to the stack itself. (These seem to be the same as the Chern classes in "delocalized cohomology" described in e.g. [110 112, though our starting point is different.)

Intuitively, the idea is that on any component of the inertia stack determined by some generic automorphism, the bundle should decompose into eigenbundles, and $\chi$ is the eigenvalue associated with the action of that automorphism on the bundle. Slightly more generally, one can define a "diagonalization map"

$$
d: K^{0}\left(I_{\mathfrak{X}}\right) \otimes \mathbb{C} \longrightarrow K^{0}\left(I_{\mathfrak{X}}\right) \otimes \mathbb{C}
$$

which on a component $\alpha$ maps a sheaf $\mathcal{F}$ to its isotypic decomposition, weighted by characters:

$$
\left.d([\mathcal{F}])\right|_{\alpha}=\sum_{\chi} \mathcal{F}_{\alpha, \chi} \otimes \chi .
$$


In this language,

$$
\operatorname{ch}^{\mathrm{rep}}(V)=\operatorname{ch}\left(d\left(q^{*} V\right)\right) .
$$

To clarify these ideas, let us work through some examples.

First, we shall consider a vector bundle on a trivial gerbe. Consider a vector bundle $V \rightarrow \mathfrak{X} \equiv X \times B \mathbb{Z}_{k}$, so $V=p_{1}^{*} E \otimes p_{2}^{*} \zeta$ for some bundle $E \rightarrow$ $X$ and representation $\zeta \in \mathbb{Z}_{k}^{\vee}$.

The inertia stack $I_{\mathfrak{X}}$ is given by

$$
I_{\mathfrak{X}}=\coprod_{g \in \mathbb{Z}_{k}} X \times B \mathbb{Z}_{k} \times\{g\} .
$$

There is a forgetful map $q: I_{\mathfrak{X}} \rightarrow X \times B \mathbb{Z}_{k}$.

Consider

$$
q^{*} V=\oplus_{\chi \in \mathbb{Z}_{k}^{\vee}} V_{\chi},
$$

where $V_{\chi}$ is the $\chi$ eigenspace for the $g$ action on $q^{*} V$ :

$$
\begin{aligned}
\left.q^{*} V\right|_{X \times B \mathbb{Z}_{k} \times\{g\}} & =V, \\
\left.V_{\chi}\right|_{X \times B \mathbb{Z}_{k} \times\{g\}} & = \begin{cases}V & \text { if } \chi(g)=\zeta(g), \\
0 & \text { else. }\end{cases}
\end{aligned}
$$

Now, we want to compute $\operatorname{ch}^{\mathrm{rep}}(V) \in H^{\bullet}\left(I_{\mathfrak{X}}, \mathbb{C}\right)$.

$$
V \mapsto q^{*} V=\oplus_{\chi} V_{\chi} \mapsto \oplus_{\chi} V_{\chi} \otimes \chi
$$

where $V_{\chi} \otimes \chi \in K^{0}\left(I_{\mathfrak{X}}\right) \otimes \mathbb{C}$. (We think of $V_{\chi} \in K^{0}\left(I_{\mathfrak{X}}\right)$, and $\chi \in \mathbb{C}$.)

Then,

$$
\begin{array}{r}
\operatorname{ch}^{\mathrm{rep}}(V)=\operatorname{ch}\left(\oplus_{\chi} V_{\chi} \otimes \chi\right) \in H^{\bullet}\left(I_{\mathfrak{X}}, \mathbb{C}\right)=\oplus_{g} H^{\bullet}(X), \\
\left.V_{\chi} \otimes \chi\right|_{X \times B \mathbb{Z}_{k} \times\{g\}}= \begin{cases}V \otimes \chi & \text { if } \chi(g)=\zeta(g), \\
0 & \text { else. }\end{cases}
\end{array}
$$

Putting this together, we find

$$
\operatorname{ch}^{\mathrm{rep}}(V)=\left(\left.\operatorname{ch}^{\mathrm{rep}}(V)\right|_{(g)}\right)_{g \in \mathbb{Z}_{k}},
$$

where

$$
\left.\operatorname{ch}^{\mathrm{rep}}(V)\right|_{(g)}=\oplus_{\chi \text { s.t. } \chi(g)=\zeta(g)} \operatorname{ch}(V) \otimes \chi .
$$

Similarly,

$$
\left.\operatorname{ch}^{\text {rep }}(T \mathfrak{X})\right|_{(g)}=\oplus_{\chi \text { s.t. } \chi(g)=1} \operatorname{ch}(T \mathfrak{X}) \otimes \chi .
$$


For $g=1$,

$$
\left.\operatorname{ch}^{\mathrm{rep}}(V)\right|_{(1)}=\oplus_{\chi} \operatorname{ch}(V) \otimes \chi
$$

and similarly for $\operatorname{ch}^{\mathrm{rep}}(T X)^{(1)}$.

Now, suppose $k$ is prime. Then $\chi(g)=1$ implies $\chi=1$. Thus,

$$
\begin{aligned}
\left.\operatorname{ch}^{\mathrm{rep}}(V)\right|_{(g)} & =\operatorname{ch}(V) \otimes \zeta(g), \\
\left.\operatorname{ch}^{\mathrm{rep}}(T \mathfrak{X})\right|_{(g)} & =\operatorname{ch}(T \mathfrak{X}) \otimes 1,
\end{aligned}
$$

for all $g$.

Next, let us consider a line bundle on a nontrivial gerbe. Consider the prototypical example of a $\mathbb{Z}_{k}$ gerbe on $\mathbb{P}^{n}: \mathfrak{X}=\mathbb{P}_{[k, k, \ldots, k]}^{n}$. Let $\mathcal{O}_{\mathfrak{X}}(m)$ denote the holomorphic line bundle defined by $\mathbb{C}^{\times}$weight $-m$. In other words, if $m$ is divisible by $k$, then $\mathcal{O}_{\mathfrak{X}}(m)$ is the pullback of $\mathcal{O}_{\mathbb{P}^{n}}(m / k)$ under the projection map from the gerbe $\mathfrak{X}$ to the underlying space $\mathbb{P}^{n}$.

The components of the inertia stack are labelled by $k$ th roots of unity (not characters, but group elements). The Chern classes ch $^{\text {rep }}$ have $k$ components, each component in a cohomology class (with complex coefficients) on the stack. If we let $\alpha$ denote a $k$ th root of unity, then on that component of the inertia stack,

$$
\left.c_{1}^{\mathrm{rep}}\left(\mathcal{O}_{\mathfrak{X}}(m)\right)\right|_{\alpha}=\frac{m}{k} \alpha^{-m} J
$$

where $J$ is the pullback to the gerbe of the hyperplane class, and the total Chern character is

$$
\left.\operatorname{ch}^{\mathrm{rep}}\left(\mathcal{O}_{\mathfrak{X}}(m)\right)\right|_{\alpha}=\alpha^{-m} \exp \left(\frac{m}{k} J\right) .
$$

To derive this, remember that for a line bundle $L$ over the stack $\mathfrak{X}$, if $\pi$ : $I_{\mathfrak{X}} \rightarrow \mathfrak{X}$ denotes the projection from the inertia stack to $\mathfrak{X}$, then the Chern characters are

$$
\left.\operatorname{ch}^{\mathrm{rep}}(L)\right|_{\mathfrak{X} \times\{\alpha\}}=\left.\pi^{*} \operatorname{ch}(L)\right|_{\mathfrak{X} \times\{\alpha\}} \otimes \chi,
$$

where $\chi$ is the eigenvalue of the stabilizer $\alpha$ on $\left.\pi^{*} L\right|_{\mathfrak{X} \times\{\alpha\}}$. Here, $\chi=\alpha^{-m}$.

More generally, over all components, we write

$$
c_{1}^{\mathrm{rep}}\left(\mathcal{O}_{\mathfrak{X}}(m)\right)=\left(\frac{m}{k} J, \ldots, \frac{m}{k} \alpha^{-m} J, \ldots\right) .
$$

Multiplication of components of $\mathrm{ch}^{\mathrm{rep}}$ multiplies not only the cohomology classes, but also the coefficients. For example,

$$
\left(\left.c_{1}^{\mathrm{rep}}(\mathcal{O}(m))\right|_{\mathfrak{X} \times\{\alpha\}}\right)^{2}=\left(\frac{m}{k} J\right)^{2} \alpha^{-2 m} .
$$


Now, for a line bundle $L$ on an ordinary space,

$$
\operatorname{ch}_{2}(L)=(1 / 2) c_{1}^{2}(L)
$$

but here, by contrast,

$$
\begin{aligned}
\left.\operatorname{ch}_{2}^{\text {rep }}(\mathcal{O}(m))\right|_{\mathfrak{X} \times\{\alpha\}} & =\frac{1}{2}\left(\frac{m}{k} J\right)^{2} \alpha^{-m} \\
& =\alpha^{+m} \frac{1}{2}\left(\left.c_{1}^{\text {rep }}(\mathcal{O}(m))\right|_{\mathfrak{X} \times\{\alpha\}}\right)^{2}
\end{aligned}
$$

so that the usual relation between Chern classes and Chern characters is modified on a stack. (In fact, if we were computing Chern classes of a bundle that split as several different eigenbundles, the relation would be much more complicated than just an additional complex phase.)

As a consistency check, let us compute the index of this line bundle, using Hirzebruch-Riemann-Roch. For any bundle $\mathcal{E} \rightarrow \mathfrak{X}$, the HirzebruchRiemann-Roch index theorem says

$$
\chi(\mathcal{E})=\int_{I_{\mathfrak{X}}} \operatorname{ch}^{\mathrm{rep}}(\mathcal{E}) \operatorname{Td}(\mathfrak{X})
$$

where

$$
\chi(\mathcal{E})=\sum_{i}(-)^{i} h^{i}(\mathfrak{X}, \mathcal{E})
$$

and

$$
\operatorname{Td}(\mathfrak{X})=\alpha_{\mathfrak{X}}^{-1} \operatorname{Td}\left(T I_{\mathfrak{X}}\right),
$$

where

$$
\alpha_{\mathfrak{X}}=\operatorname{ch}\left(d\left(\lambda_{q}\right)\right), \quad \lambda_{q}=\sum_{k}(-)^{k} \wedge^{k} N_{q}^{*},
$$

for $N_{q}$ the normal bundle. (As $\lambda_{q}$ is not a pullback from $\mathfrak{X}$, but rather is defined intrinsically on $I_{\mathfrak{X}}, \operatorname{ch}^{\mathrm{rep}}\left(\lambda_{q}\right)$ is not well-defined, so instead the pertinent Chern character is defined via the diagonalization map $d$.)

In the present case, since each component of the inertia stack $I_{\mathfrak{X}}$ is isomorphic to the original stack $\mathfrak{X}$, the normal bundle $N_{q}$ vanishes, and each component of $\operatorname{ch}\left(d\left(\lambda_{q}\right)\right)$ is 1 . Furthermore, as $\mathfrak{X}$ is essentially a $k$-fold 
quotient of $\mathbb{P}^{n}$,

$$
\int_{\mathfrak{X}}=\frac{1}{k} \int_{\mathbb{P}^{n}} .
$$

Plugging into the index formula,

$$
\begin{aligned}
\int_{I_{\mathfrak{X}}} \operatorname{ch}^{\mathrm{rep}}\left(\mathcal{O}_{X}(m)\right) \operatorname{Td}\left(T I_{\mathfrak{X}}\right) & =\sum_{\alpha} \int_{\mathfrak{X}} \alpha^{-m} \operatorname{ch}\left(\mathcal{O}_{\mathfrak{X}}(m)\right) \operatorname{Td}(T \mathfrak{X}), \\
& =\sum_{\alpha} \alpha^{-m} \int_{\mathfrak{X}} \sum_{i} \operatorname{ch}_{i}\left(\mathcal{O}_{\mathfrak{X}}(m)\right) \operatorname{Td}_{n-i}(T \mathfrak{X}) .
\end{aligned}
$$

Now, since $\alpha$ is a $k$ th root of unity, the sum

$$
\sum_{\alpha} \alpha^{-m}
$$

will vanish unless $m$ is divisible by $k$. Thus, if $m$ is not divisible by $k$, we find that $\chi\left(\mathcal{O}_{\mathfrak{X}}(m)\right)$ vanishes. Next, suppose that $m=n k$ for some integer $n$. Then,

$$
\begin{aligned}
\int_{I_{\mathfrak{X}}} \operatorname{ch}^{\mathrm{rep}}\left(\mathcal{O}_{\mathfrak{X}}(m)\right) \operatorname{Td}\left(I_{\mathfrak{X}}\right) & =\sum_{\alpha} \int_{\mathfrak{X}} \alpha^{-m} \operatorname{ch}\left(\mathcal{O}_{\mathfrak{X}}(m)\right) \operatorname{Td}(T \mathfrak{X}) \\
& =\sum_{\alpha} \int_{\mathfrak{X}} \pi^{*} \operatorname{ch}\left(\mathcal{O}_{\mathbb{P}^{n}}(n)\right) \operatorname{Td}\left(T \mathbb{P}^{n}\right) \\
& =\sum_{\alpha} \frac{1}{k} \int_{\mathbb{P}^{n}} \operatorname{ch}\left(\mathcal{O}_{\mathbb{P}^{n}}(n)\right) \operatorname{Td}\left(T \mathbb{P}^{n}\right) \\
& =\int_{\mathbb{P}^{n}} \operatorname{ch}\left(\mathcal{O}_{\mathbb{P}^{n}}(n)\right) \operatorname{Td}\left(T \mathbb{P}^{n}\right) \\
& =\chi\left(\mathbb{P}^{n}, \mathcal{O}_{\mathbb{P}^{n}}(n)\right)
\end{aligned}
$$

Now, let us compare to expectations. In the present case, if $m$ is not divisible by $k$, then all the sheaf cohomology groups of $\mathcal{O}_{\mathfrak{X}}(m)$ should vanish, so the Euler class $\chi\left(\mathcal{O}_{\mathfrak{X}}(m)\right)$ should vanish, exactly as we have computed. If $m$ is divisible by $k$, then $\chi\left(\mathcal{O}_{\mathfrak{X}}(m)\right)=\chi\left(\mathcal{O}_{\mathbb{P}^{n}}(m / k)\right)$, again matching the result of the computation.

Another example ${ }^{26}$ will be handy to understand.

${ }^{26}$ We would like to thank T. Pantev for explaining this example to us. 
Take $\mathfrak{X}=\left[T^{4} / \mathbb{Z}_{2}\right]$, where the $\mathbb{Z}_{2}$ acts by sign flips (and so has 16 fixed points). Let us compute

$$
\chi\left(\mathcal{O}_{\mathfrak{X}}[0]\right), \quad \chi\left(\mathcal{O}_{\mathfrak{X}}[1 / 2]\right),
$$

where $\mathcal{O}_{\mathfrak{X}}[0]$ denotes the structure sheaf with trivial $\mathbb{Z}_{2}$-equivariant structure, and $\mathcal{O}_{\mathfrak{X}}[1 / 2]$ denotes the structure sheaf with nontrivial equivariant structure. For this $\mathfrak{X}, I_{\mathfrak{X}}$ has 17 components: one copy of $\mathfrak{X}$, and 16 copies of $\left[\mathrm{pt} / \mathbb{Z}_{2}\right]$. From the definition

$$
\left.\operatorname{ch}^{\mathrm{rep}}(L)\right|_{\alpha}=\left.\pi^{*} \operatorname{ch}(L)\right|_{\alpha} \otimes \chi
$$

where $\alpha$ is a component of $I_{\mathfrak{X}}$ and $\chi$ the eigenvalue of $\alpha$ 's stabilizer on $\pi^{*} L$, it is straightforward to compute that

$$
\begin{aligned}
\operatorname{ch}^{\mathrm{rep}}(\mathcal{O}[0]) & =(1, \overrightarrow{0}, 0 ; 1, \ldots, 1), \\
\operatorname{ch}^{\mathrm{rep}}(\mathcal{O}[1 / 2]) & =(1, \overrightarrow{0}, 0 ;-1, \ldots,-1),
\end{aligned}
$$

where the leading three entries are for the $\mathfrak{X}$ component, corresponding to elements of $H^{0}(\mathfrak{X})=\mathbb{C}, H^{2}(\mathfrak{X})=\mathbb{C}^{6}, H^{4}(\mathfrak{X})=\mathbb{C}$, respectively, and the remaining sixteen entries are each for a copy of $\left[\mathrm{pt} / \mathbb{Z}_{2}\right]$.

The normal bundle $N$ is 0 for the trivial component $\left[T^{4} / \mathbb{Z}_{2}\right]$ of $I_{\mathfrak{X}}$, and is $\mathbb{C}^{2}$ with $\mathbb{Z}_{2}$ acting by sign flips for the other components of $I_{\mathfrak{X}}$. From that, we read off that

$$
\begin{aligned}
\operatorname{ch}\left(d\left(\wedge^{0} N\right)\right) & =(1, \overrightarrow{0}, 0 ; 1, \ldots, 1) \\
\operatorname{ch}(d(N)) & =(0, \overrightarrow{0}, 0 ;-2, \ldots,-2), \\
\operatorname{ch}\left(d\left(\wedge^{2} N\right)\right) & =(0, \overrightarrow{0}, 0 ; 1, \ldots, 1) \\
\operatorname{ch}\left(d\left(\wedge^{k} N\right)\right) & =0 \quad \text { for } k>2 .
\end{aligned}
$$

From this we find

$$
\alpha_{\mathfrak{X}}=\operatorname{ch}\left(d\left(\lambda_{q}\right)\right)=\operatorname{ch}\left(d\left(\sum_{i}(-)^{i} \wedge^{i} N^{*}\right)\right)=(1, \overrightarrow{0}, 0 ; 4, \ldots, 4) .
$$

In addition,

$$
\operatorname{ch}^{\mathrm{rep}}\left(\operatorname{Td}\left(T I_{\mathfrak{X}}\right)\right)=(1, \overrightarrow{0}, 0 ; 1, \ldots, 1)
$$

hence

$$
\operatorname{Td}(\mathfrak{X})=\alpha_{\mathfrak{X}}^{-1} \operatorname{Td}\left(T I_{\mathfrak{X}}\right)=(1, \overrightarrow{0}, 0 ; 1 / 4, \ldots, 1 / 4) .
$$


Putting this together, we find

$$
\begin{aligned}
\chi\left(\mathcal{O}_{\mathfrak{X}}[0]\right) & =\int_{I_{\mathfrak{X}}} \operatorname{ch}^{\mathrm{rep}}\left(\mathcal{O}_{\mathfrak{X}}[0]\right) \operatorname{Td}(\mathfrak{X}) \\
& =\int_{\left[T^{4} / \mathbb{Z}_{2}\right]}(1)(1)+16 \int_{\left[\mathrm{pt} / \mathbb{Z}_{2}\right]}(1)(1 / 4), \\
& =0+4 \int_{\left[\mathrm{pt} / \mathbb{Z}_{2}\right]} 1, \\
& =4\left(\frac{1}{2}\right)=2, \\
\chi\left(\mathcal{O}_{\mathfrak{X}}[1 / 2]\right) & =\int_{I_{\mathfrak{X}}} \operatorname{ch}^{\mathrm{rep}}\left(\mathcal{O}_{\mathfrak{X}}[1 / 2]\right) \operatorname{Td}(\mathfrak{X}) \\
& =\int_{\left[T^{4} / \mathbb{Z}_{2}\right]}(1)(1)+16 \int_{\left[\mathrm{pt} / \mathbb{Z}_{2}\right]}(-1)(1 / 4), \\
& =0-4 \int_{\left[\mathrm{pt} / \mathbb{Z}_{2}\right]} 1, \\
& =-4\left(\frac{1}{2}\right)=-2 .
\end{aligned}
$$

Let $Y$ denote a minimal resolution of $T^{4} / \mathbb{Z}_{2}$. Applying the McKay correspondence [113], it can be shown [89] that the bundle $\mathcal{O}_{\mathfrak{X}}[0]$ maps to $\mathcal{O}_{Y}$, and $\mathcal{O}_{\mathfrak{X}}[1 / 2]$ maps to $\mathcal{O}_{Y}\left(-(1 / 2) \sum E_{a}\right)$ where the $E_{a}$ are the exceptional divisors. Furthermore, it can be shown that on $Y, \chi\left(\mathcal{O}_{Y}\right)=+2$ and $\chi\left(\mathcal{O}_{Y}\left(-(1 / 2) \sum E_{a}\right)\right)=-2$, matching the Euler characteristics above.

So far we have discussed the index of the operator $\bar{\partial}$. We are not aware of rigorous results concerning the Dirac index, which would be of direct relevance for physics. That said, it is very natural to conjecture that, by analogy with smooth manifolds, the Dirac index is computed by a closely analogous expression, except that $\operatorname{Td}\left(T I_{\mathfrak{X}}\right)$ is replaced by

$$
\operatorname{Td}\left(T I_{\mathfrak{X}}\right) \exp \left(-\frac{1}{2} c_{1}^{\mathrm{rep}}\left(T I_{\mathfrak{X}}\right)\right),
$$

following the usual pattern that

$$
\hat{A}(M)=\operatorname{Td}(M) \exp \left(-(1 / 2) c_{1}(M)\right)
$$

for a smooth manifold $M$.

See also e.g. [114 116] and references therein for more information on index theorems on stacks. 


\section{Appendix D. Roots of canonical bundles}

On a $\mathbb{Z}_{k}$ gerbe, sometimes there exist $k$ th roots of the canonical bundle, and sometimes not, depending upon the gerbe. Let us work through some examples.

First, consider a nontrivial $\mathbb{Z}_{k}$ gerbe over $\mathbb{P}^{1}$. In particular, let us consider the gerbe defined by the quotient

$$
\frac{\mathbb{C}^{2}-0}{\mathbb{C}^{\times}}
$$

where the $\mathbb{C}^{\times}$acts with weight $k$. We will show that the pullback of any line bundle on $\mathbb{P}^{1}$ to this gerbe does admit a $k$ th root.

A line bundle over this gerbe will have a total space of the form

$$
\frac{\left(\mathbb{C}^{2}-0\right) \times \mathbb{C}}{\mathbb{C}^{\times}}
$$

where $\mathbb{C}^{\times}$acts on $([x, y], z)$ as

$$
([x, y], z) \mapsto\left(\left[\lambda^{k} x, \lambda^{k} y\right], \lambda^{n} z\right)
$$

and $n$ classifies the line bundle. The pullback of $\mathcal{O}(m)$ on $\mathbb{P}^{1}$ to the gerbe has $n=k m$, so a line bundle on the gerbe with $n=m$ has the property that its $k$ th tensor power with itself is the pullback of $\mathcal{O}(m)$.

Thus, on this $\mathbb{Z}_{k}$ gerbe, $k$ th roots of pullbacks of any line bundle on the base space do exist.

Next, let us consider the trivial $\mathbb{Z}_{k}$ gerbe over $\mathbb{P}^{1}$. Here, the total space any line bundle over this gerbe can be described as

$$
\frac{(\operatorname{Tot} L) \times \mathbb{C}^{\times}}{\mathbb{C}^{\times}}
$$

where $L$ is a line bundle on $\mathbb{P}^{1}$, and the $\mathbb{C}^{\times}$acts only on $\mathbb{C}^{\times}$. Here, there is clearly no way to construct a $k$ th root of $L$ (unless $L$ already had a $k$ th root on $\left.\mathbb{P}^{1}\right)$.

\section{References}

[1] R. Donagi, Y. -H. He, B. A. Ovrut and R. Reinbacher, Moduli dependent spectra of heterotic compactifications. Phys. Lett., B598 (2004), 279-284, arXiv:hep-th/0403291. 
[2] R. Donagi, Y.-H. He, B. A. Ovrut and R. Reinbacher, The particle spectrum of heterotic compactifications. JHEP, 0412 (2004), 054, arXiv:hep-th/0405014.

[3] R. Donagi, Y.-H. He, B. A. Ovrut and R. Reinbacher, Higgs doublets, split multiplets and heterotic $S U(3)(C) \times S U(2)(L) \times U(1)(Y)$ spectra. Phys. Lett., B618 (2005), 259-264, arXiv:hep-th/0409291.

[4] R. Donagi, Y. -H. He, B. A. Ovrut and R. Reinbacher, The spectra of heterotic standard model vacua. JHEP, 0506 (2005), 070, arXiv: hep-th/0411156.

[5] V. Bouchard and R. Donagi, An SU(5) heterotic standard model. Phys. Lett., B633 (2006), 783-791, arXiv:hep-th/0512149.

[6] V. Braun, Y.-H. He, B. A. Ovrut and T. Pantev, A heterotic standard model. Phys. Lett., B618 (2005), 252-258, arXiv:hep-th/0501070.

[7] V. Braun, Y.-H. He, B. A. Ovrut and T. Pantev, A standard model from the $E(8) \times E(8)$ heterotic superstring. JHEP, 0506 (2005), 039, arXiv:hep-th/0502155.

[8] V. Braun, Y.-H. He, B. A. Ovrut and T. Pantev, Vector bundle extensions, sheaf cohomology, and the heterotic standard model. Adv. Theor. Math. Phys., 10 (2006), 525-589, arXiv: hep-th/0505041.

[9] L. B. Anderson, J. Gray, Y.-H. He and A. Lukas, Exploring positive monad bundles and a new heterotic standard model. JHEP, 1002 (2010), 054, arXiv:0911.1569.

[10] L. B. Anderson, J. Gray, A. Lukas and E. Palti, Two hundred heterotic standard models on smooth Calabi-Yau threefolds. Phys. Rev., D84 (2011), 106005, arXiv:1106.4804.

[11] L. B. Anderson, J. Gray, A. Lukas and E. Palti, Heterotic line bundle standard models. JHEP, 1206 (2012), 113, arXiv:1202.1757.

[12] V. Braun, P. Candelas, R. Davies and R. Donagi, The MSSM spectrum from $(0,2)$-deformations of the heterotic standard embedding. JHEP, 1205 (2012), 127, arXiv:1112.1097.

[13] S. Katz, T. Pantev and E. Sharpe, D-branes, orbifolds, and Ext groups. Nucl. Phys., B673 (2003), 263-300, arXiv: hep-th/0212218.

[14] T. Pantev and E. Sharpe, Notes on gauging noneffective group actions. arXiv: hep-th/0502027. 
[15] T. Pantev and E. Sharpe, String compactifications on Calabi-Yau stacks. Nucl. Phys., B733 (2006), 233-296, arXiv:hep-th/0502044.

[16] T. Pantev, E. Sharpe, GLSM's for gerbes (and other toric stacks). Adv. Theor. Math. Phys., 10 (2006), 77-121, arXiv:hep-th/0502053.

[17] S. Hellerman, A. Henriques, T. Pantev, E. Sharpe and M. Ando, Cluster decomposition, T-duality, and gerby CFT's. Adv. Theor. Math. Phys., 11 (2007), 751-818, arXiv:hep-th/0606034.

[18] A. Caldararu, J. Distler, S. Hellerman, T. Pantev and E. Sharpe, Non-birational twisted derived equivalences in abelian GLSMs. Comm. Math. Phys., 294 (2010), 605-645, arXiv:0709.3855.

[19] R. Karp, On the $\mathbf{C}^{n} / \mathbf{Z}_{m}$ fractional branes. J. Math. Phys., 50 (2009), 022304, arXiv:hep-th/0602165.

[20] C. Herzog and R. Karp, On the geometry of quiver gauge theories: Stacking exceptional collections. arXiv:hep-th/0605177.

[21] E. Sharpe, Derived categories and stacks in physics. Contribution to the proceedings of the ESI research conference on homological mirror symmetry (Vienna, Austria, June 2006), arXiv: hep-th/0608056.

[22] E. Sharpe, Landau-Ginzburg models, gerbes, and Kuznetsov's homological projective duality. To appear in the proceedings of Topology, $\mathbf{C}^{*}$ algebras, string duality (Texas Christian University, May 18-22, 2009).

[23] E. Sharpe, GLSM's, gerbes, and Kuznetsov's homological projective duality. Contribution to the proceedings of Quantum theory and symmetries 6, arXiv:1004.5388.

[24] K. Hori, Duality in two-dimensional $(2,2)$ supersymmetric non-abelian gauge theories. arXiv:1104.2853.

[25] N. Addington, E. Segal and E. Sharpe, D-brane probes, branched double covers, and noncommutative resolutions. arXiv:1211.2446.

[26] E. Sharpe, Predictions for Gromov-Witten invariants of noncommutative resolutions. arXiv:1212.5322.

[27] J. Halverson, V. Kumar and D. Morrison, New methods for characterizing phases of $2 d$ supersymmetric gauge theories. arXiv:1305.3278.

[28] E. Sharpe, A few Ricci-flat stacks as phases of exotic GLSMs. arXiv: 1306.5440. 
[29] E. Andreini, Y. Jiang and H.-H. Tseng, On Gromov-Witten theory of root gerbes. arXiv:0812.4477.

[30] E. Andreini, Y. Jiang and H.-H. Tseng, Gromov-Witten theory of product stacks. arXiv:0905.2258.

[31] E. Andreini, Y. Jiang and H.-H. Tseng, Gromov-Witten theory of etale gerbes, $i$ : root gerbes. arXiv:0907.2087.

[32] H.-H. Tseng, On degree zero elliptic orbifold Gromov-Witten invariants. arXiv:0912.3580.

[33] A. Gholampour and H.-H. Tseng, On Donaldson-Thomas invariants of threefold stacks and gerbes. arXiv:1001.0435.

[34] X. Tang and H.-H. Tseng, Duality theorems of étale gerbes on orbifolds. arXiv: 1004.1376.

[35] K. Dienes and B. Thomas, On the inconsistency of Fayet-Iliopoulos terms in supergravity theories. Phys. Rev., D81 (2010), 065023, arXiv:0911.0677.

[36] N. Seiberg, Modifying the sum over topological sectors and constraints on supergravity. arXiv:1005.0002.

[37] J. Distler and E. Sharpe, Quantization of Fayet-Iliopoulos parameters in supergravity. Phys. Rev., D83 (2011), 085010, arXiv:1008.0419.

[38] T. Banks and N. Seiberg, Symmetries and strings in field theory and gravity. arXiv: 1011.5120 .

[39] S. Hellerman and E. Sharpe, Sums over topological sectors and quantization of Fayet-Iliopoulos parameters. Adv. Theor. Math. Phys., 15 (2011), 1141-1199, arXiv:1012.5999.

[40] E. Witten and J. Bagger, Quantization of Newton's constant in certain supergravity thoeries. Phys. Lett., B115 (1982), 202-206.

[41] B. Jia and E. Sharpe, Rigidly supersymmetric gauge theories on curved superspace. JHEP, 1204 (2012), 139, arXiv:1109.5421.

[42] M. Berasaluce-González, P. G. Cámara, F. Marchesano, D. Regalado and A. M. Uranga, Non-abelian discrete gauge symmetries in $4 d$ string models. JHEP, 1209 (2012), 059, arXiv:1206.2383.

[43] M. Berasaluce-González, P. G. Cámara, F. Marchesano and A. M. Uranga, $Z p$ charged branes in flux compactifications. JHEP, 1304 (2013), 138, arXiv:1211.5317. 
[44] W.-M. Chen and Y.-B. Ruan, A new cohomology theory for orbifold. Comm. Math. Phys., 248 (2004), 1-31, arXiv:math/0004129.

[45] D. Abramovich, T. Graber and A. Vistoli, Gromov-Witten theory of Deligne-Mumford stacks. Amer. J. Math., 130 (2008), 1337-1398, arXiv:math.AG/0603151.

[46] T. Coates, A. Corti, Y. Lee and H. Tseng, The quantum orbifold cohomology of weighted projective spaces. Acta. Math., 202 (2009), 139-193, arXiv:math.AG/0608481.

[47] E. Mann, Orbifold quantum cohomology of weighted projective spaces. J. Alg. Geom., 17 (2008), 137-166, arXiv:math.AG/0610965.

[48] A. Vistoli, Intersection theory on algebraic stacks and on their moduli spaces. Inv. Math., 97 (1989), 613-670.

[49] T. Gomez, Algebraic stacks. Proc. Indian Acad. Sci. Math. Sci., 111 (2001), 1-31, arXiv:math.AG/9911199.

[50] G. Laumon and L. Moret-Bailly, Champs algébriques. Springer, 1999.

[51] K. Behrend and P. Xu, Differentiable stacks and gerbes. J. Sympl. Geom., 9 (2011), 285-341, arXiv:math/0605694.

[52] J. Heinloth, Notes on differentiable stacks, Mathematisches Institut, Georg-August-Universität Göttingen, Seminars Winter Term 2004/ 2005, 1-32, Universitätsdrucke Göttingen, Göttingen, 2005, available at http://www . uni-due.de/ hm0002/stacks.pdf.

[53] D. Metzler, Topological and smooth stacks. arXiv:math/0306176.

[54] B. Noohi, Foundations of topological stacks I. arXiv:math/0503247.

[55] B. Noohi, Fundamental groups of topological stacks with slice property. arXiv:0710.2615.

[56] B. Noohi, Homotopy types of topological stacks. arXiv:0808.3799.

[57] J. Heinloth, Twisted Chern classes and $\mathbf{G}_{m}$ gerbes. C. R. Math. Acad. Sci. Paris, 341 (2005), 623-626.

[58] U. Bunke, T. Schick and M. Spitzweck, Sheaf theory for stacks in manifolds and twisted cohomology for $S^{1}$-gerbes. Algebr. Geom. Topol., 7 (2007), 1007-1062.

[59] D. Freed and C. Vafa, Global anomalies on orbifolds. Comm. Math. Phys., 110 (1987), 349-389. 
[60] K. Dienes, private communication.

[61] H. Kawai, D. Lewellen, J. Schwartz and S. Tye, The spin structure construction of string models and multiloop modular invariance. Nucl. Phys., B299 (1988), 431-470.

[62] N. Hitchin, Lectures on special Lagrangian submanifolds. arXiv: math.DG/9907034.

[63] A. Caldararu, S. Katz and E. Sharpe, D-branes, B fields, and Ext groups. Adv. Theor. Math. Phys., 7 (2004), 381-404, arXiv:hep-th/ 0302099.

[64] P. Ginsparg, On toroidal compactification of heterotic superstrings. Phys. Rev., D35 (1987), 648-654.

[65] J. Distler and B. Greene, Aspects of $(2,0)$ string compactifications. Nucl. Phys., B304 (1988), 1-62.

[66] J. F. Adams, Lectures on exceptional Lie groups. University of Chicago Press, 1996.

[67] J. Distler and E. Sharpe, Heterotic compactifications with principal bundles for general groups and general levels. Adv. Theor. Math. Phys., 14 (2010), 335-398, arXiv:hep-th/0701244.

[68] S. J. Gates, Jr. and W. Siegel, Leftons, rightons, nonlinear sigma models, and superstrings. Phys. Lett., B206 (1988), 631-638.

[69] D. Depireux, S. J. Gates, Jr. and Q-H. Park, Lefton-righton formulation of massless Thirring models. Phys. Lett., B224 (1989), 364-372.

[70] S. Bellucci, D. Depireux and S. J. Gates, Jr., (1,0) Thirring models and the coupling of spin-zero fields to the heterotic string. Phys. Lett., B232 (1989), 67-74.

[71] S. J. Gates, Jr., S. Ketov, S. Kuzenko and O. Soloviev, Lagrangian chiral coset construction of heterotic string theories in $(1,0)$ superspace. Nucl. Phys., B362 (1991), 199-231.

[72] S. J. Gates, Jr., Strings, superstrings, and two-dimensional lagrangian field theory. In: Functional integration, geometry, and strings (Z. Haba and J. Sobczyk eds.), proceedings of the XXV Winter School of Theoretical Physics, pp. 140-184, Karpacz, Poland (Feb. 1989), Birkhäuser, 1989 . 
[73] R. Slansky, Group theory for unified model building. Phys. Rept., 79 (1981), 1-128.

[74] S. Kachru and E. Witten, Computing the complete massless spectrum of a Landau-Ginzburg orbifold. Nucl. Phys., B407 (1993), 637-666, arXiv:hep-th/9307038.

[75] J. Distler and S. Kachru, (0,2) Landau-Ginzburg theory. Nucl. Phys., B413 (1994), 213-243, arXiv:hep-th/9309110.

[76] L. Dixon and J. Harvey, String theories in ten dimensions without spacetime supersymmetry. Nucl. Phys., B274 (1986), 93-105.

[77] H. Kawai, D. Lewellen and H. Tye, Classification of closed fermionic string models. Phys. Rev., D34 (1986), 3794-3804.

[78] J. Polchinski, String theory, Volume 2. Cambridge University Press, 2005.

[79] G. Aldazabal, A. Font, L. Ibanez and A. Uranga, Nonperturbative heterotic $D=6, D=4, N=1$ orbifold vacua. Nucl. Phys., B519 (1998), 239-281, arXiv:hep-th/9706158.

[80] G. Aldazabal, A. Font, L. Ibanez, A. Uranga and G. Violero, Giving up modular invariance constraints in heterotic orbifold vacua. Nucl. Phys. Proc. Suppl., 68 (1998), 128-139.

[81] R. Donagi, B. Ovrut, T. Pantev and R. Reinbacher, SU(4) instantons on Calabi-Yau threefolds with $\mathbf{Z}_{2} \times \mathbf{Z}_{2}$ fundamental group. arXiv: hep-th/0307273.

[82] R. Donagi, B. A. Ovrut, T. Pantev and D. Waldram, Standard model bundles on nonsimply connected Calabi-Yau threefolds. JHEP, 0108 (2001), 053, arXiv:hep-th/0008008.

[83] R. Donagi, B. A. Ovrut, T. Pantev and D. Waldram, Standard model bundles. Adv. Theor. Math. Phys., 5 (2002), 563-615, arXiv:math/ 0008010 [math-ag].

[84] R. Donagi, B. A. Ovrut, T. Pantev and D. Waldram, Spectral involutions on rational elliptic surfaces. Adv. Theor. Math. Phys., 5 (2002) 499-561, arXiv:math/0008011 [math-ag].

[85] B. A. Ovrut, T. Pantev and R. Reinbacher, Torus fibered Calabi-Yau threefolds with nontrivial fundamental group. JHEP, 0305 (2003), 040, arXiv: hep-th/0212221. 
[86] B. A. Ovrut, T. Pantev and R. Reinbacher, Invariant homology on standard model manifolds. JHEP, 0401 (2004), 059, arXiv:hep-th/ 0303020.

[87] V. Braun, B. A. Ovrut, T. Pantev and R. Reinbacher, Elliptic CalabiYau threefolds with $Z(3) \times Z(3)$ Wilson lines. JHEP, 0412 (2004), 062, arXiv:hep-th/0410055.

[88] P. Candelas and R. Davies, New Calabi-Yau manifolds with small Hodge numbers. arXiv:0809.4681.

[89] T. Pantev, private communication.

[90] A. Kapustin, Holomorphic reduction of $\mathcal{N}=2$ gauge theories, Wilson't Hooft operators, and S-duality. arXiv:hep-th/0612119.

[91] A. Kapustin and E. Witten, Electric-magnetic duality and the geometric Langlands program. arXiv:hep-th/0604151.

[92] R. Manion, Additive parts of heterotic analogues of Chen-Ruan cohomology. To appear.

[93] M. Ando and E. Sharpe, Elliptic genera of Landau-Ginzburg models over nontrivial spaces. arXiv:0905.1285.

[94] J. Distler, Notes on $N=2$ sigma models. In: Trieste 1992, Proceedings, String theory and quantum gravity '92, pp. 234-256, Princeton University Press, arXiv:hep-th/9212062.

[95] M. Bershadsky, S. Cecotti, H. Ooguri and C. Vafa, Kodaira-Spencer theory of gravity and exact results for quantum string amplitudes. Comm. Math. Phys., 165 (1994), 311-428, arXiv:hep-th/9309140.

[96] H. B. Lawson and M.-L. Michelsohn, Spin geometry. Princeton University Press, 1990.

[97] S. Katz and E. Sharpe, D-branes, open string vertex operators, and Ext groups. Adv. Theor. Math. Phys., 6 (2003), 979-1030, arXiv: hep-th/0208104.

[98] E. Sharpe, Lectures on D-branes and sheaves. arXiv:hep-th/0307245.

[99] S. Katz and E. Sharpe, Notes on certain $(0,2)$ correlation functions. Comm. Math. Phys., 262 (2006), 611-644, arXiv:hep-th/0406226.

[100] A. Adams, J. Distler and M. Ernebjerg, Topological heterotic rings. Adv. Theor. Math. Phys., 10 (2006), 657-682, arXiv:hep-th/0506 263. 
[101] E. Sharpe, Notes on certain other $(0,2)$ correlation functions. Adv. Theor. Math. Phys., 13 (2009), 33-70, arXiv:hep-th/0605005.

[102] J. McOrist and I. Melnikov, Summing the instantons in half-twisted linear sigma models. JHEP, 0902 (2009), 026, arXiv:0810.0012.

[103] R. Donagi, J. Guffin, S. Katz and E. Sharpe, A mathematical theory of quantum sheaf cohomology. arXiv:1110.3751.

[104] R. Donagi, J. Guffin, S. Katz and E. Sharpe, Physical aspects of quantum sheaf cohomology for deformations of tangent bundles of toric varieties. arXiv:1110.3752.

[105] I. Melnikov, S. Sethi and E. Sharpe, Recent developments in $(0,2)$ mirror symmetry. SIGMA, 8 (2012), 068, arXiv:1209.1134.

[106] M.-C. Tan, Two-dimensional twisted sigma models and the theory of chiral differential operators. Adv. Theor. Math. Phys., 10 (2006), 759 851, arXiv:hep-th/0604179.

[107] M.-C. Tan, Two-dimensional twisted sigma models, the mirror chiral de Rham complex, and twisted generalized mirror symmetry. JHEP, 0707 (2007), 013, arXiv:0705.0790.

[108] M. Qureshi and B. Szendröi, Calabi-Yau threefolds in weighted flag varieties. Adv. High Energy Phys., 2012 (2012), 547317, arXiv: 1105. 4282.

[109] J. Guffin and E. Sharpe, A-twisted Landau-Ginzburg models. J. Geom. Phys., 59 (2009), 1547-1580, arXiv:0801.3836.

[110] M. Atiyah and G. Segal, On equivariant Euler characteristics. J. Geom. Phys., 6 (1989), 671-677.

[111] P. Baum, J.-L. Brylinski and R. MacPherson, Cohomologie équivariante délocalisée. C. R. Acad. Sc. Paris, Série I, t., 300 (1985), 605-608.

[112] P. Baum and A. Connes, Chern character for discrete groups. In: A fête of topology: papers dedicated to Itiro Tamura (Y. Matsumoto, T. Mizutani and S. Morita eds.), pp. 163-232, Academic Press, Boston, 1988.

[113] T. Bridgeland, A. King and M. Reid, Mukai implies McKay: the McKay correspondence as an equivalence of derived categories. arXiv: math/9908027. 
[114] D. Edidin, Riemann-Roch for Deligne-Mumford stacks. arXiv: 1205. 4742.

[115] A. Buckley, M. Reid and S. Zhou, Ice cream and orbifold RiemannRoch. arXiv:1208.0457.

[116] V. Tonita and H.-H. Tseng, Quantum orbifold Hirzebruch-RiemannRoch theorem in genus zero. arXiv:1307.0262.

Department of Physics, Virginia Tech

Robeson Hall, 0435, Blacksburg, VA 24061, USA

E-mail address: lara.anderson@vt.edu

Department of Physics, The University of Texas at Austin

2515 Speedway, C1600, Austin, TX 78712-1192, USA

E-mail address: beijia@physics.utexas.edu

Department of Mathematics, David Rittenhouse Laboratory

University of Pennsylvania

209 South 33Rd Street, Philadelphia, PA 19104-6395, USA

E-mail address: rymanion@gmail.com

Department of Physics, David Rittenhouse Laboratory

University of Pennsylvania

209 South 33rd Street, Philadelphia, PA 19104-6395, USA

E-mail address: ovrut@elcapitan.hep.upenn.edu

Department of Physics, Virginia Tech

Robeson Hall, 0435, Blacksburg, VA 24061, USA

E-mail address: ersharpe@vt.edu 
